\title{
Agriculture and Wetland Applications
}

\author{
J. M. Lopez-Sanchez, J. D. Ballester-Berman, F. Vicente-Guijalba, S. R. Cloude, \\ H. McNairn, J. Shang, H. Skriver, T. Jagdhuber, I. Hajnsek, E. Pottier, \\ C. Marechal, L. Hubert-Moy, S. Corgne, S. Wdowinski, R. Touzi, G. Gosselin, \\ R. Brooks, Y. Yamaguchi, and G. Singh
}

\section{Abstract}

Based on experimental results, this chapter describes applications of SAR polarimetry to extract relevant information on agriculture and wetland scenarios by exploiting differences in the polarimetric signature of different

J. M. Lopez-Sanchez $(\varangle) \cdot$ J. D. Ballester-Berman · F. Vicente-Guijalba Institute of Computing Research, University of Alicante, Alicante,

Spain

e-mail: juanma.lopez@ua.es

S. R. Cloude

AEL Consultants, Cupar, Fife, UK

H. McNairn · J. Shang

Science and Technology Branch, Agriculture and Agri-Food Canada, Ottawa, ON, Canada

\section{H. Skriver}

Microwaves and Remote Sensing, National Space Institute, Technical University of Denmark, Lyngby, Denmark

T. Jagdhuber

Microwave and Radar Institute, German Aerospace Center (DLR),

Wessling, Germany

I. Hajnsek

Microwaves and Radar Institute, German Aerospace Center (DLR), Oberpfaffenhofen, Germany

Institute of Environmental Engineering, Swiss Federal Institute of Technology Zurich (ETH Zurich), Zürich, Switzerland

E. Pottier · C. Marechal

Institut d'Électronique et de Télécommunications de Rennes, University of Rennes-1, Rennes, France

L. Hubert-Moy $\cdot$ S. Corgne

LETG Rennes, University of Rennes-2, Rennes, France

S. Wdowinski

Division of Marine Geology and Geophysics, University of Miami, Miami, FL, USA

R. Touzi · G. Gosselin · R. Brooks

Canada Center for Remote Sensing, Natural Resources Canada, Ottawa, ON, Canada

Y. Yamaguchi · G. Singh

Institute of Science and Technology, Niigata University, Niigata, Japan scatterers, crop types and their development stage depending on their physical properties. Concerning agriculture, crop type mapping, soil moisture estimation and phenology estimation are reviewed, as they are ones with a clear benefit of full polarimetry over dual or single polarimetry. For crop type mapping, supervised or partially unsupervised classification schemes are used. Phenology estimation is treated as a classification problem as well, by regarding the different stages as different classes. Soil moisture estimation makes intensive use of scattering models, in order to separate soil and vegetation scattering and to invert for soil moisture from the isolated ground component. Then, applications of SAR polarimetry to wetland monitoring are considered that include the delineation of their extent and their characterisation by means of polarimetric decompositions. In the last section of the chapter, the use of a SAR polarimetric decomposition is shown for the assessment of the damages consequential to earthquakes and tsunamis.

\subsection{Introduction}

There exists a wide variety of remote sensing applications in the context of agriculture. A possible classification is summarised in Table 3.1, including the end-users and their motivation for such applications.

Most of these applications have made use of quad-pol data, but recently several studies have been carried out using compact polarimetry, where a single transmit (usually circular) and dual linear coherent receive is used. These have shown some promising advances in agriculture applications, for instance, combining high classification accuracy from the polarimetric information with wide swath coverage. Among all these applications, the following ones demonstrate the role of polarimetry and the benefits of quad-pol data over singlepol or dual-pol approaches: 
Table 3.1 Remote sensing applications on agriculture

\begin{tabular}{|c|c|c|}
\hline (End) users & Application(s) & Interest/motivation \\
\hline \multirow{3}{*}{$\begin{array}{l}\text { National international } \\
\text { authorities/agencies }\end{array}$} & Crop type mapping or classification & Justification of subsidies and fraud detection, acreage \\
\hline & Water resources consumption & $\begin{array}{l}\text { Control in regions suffering droughts or with scarce water } \\
\text { resources }\end{array}$ \\
\hline & Yield prediction & Economic and market predictions, price regulations, etc. \\
\hline \multirow[t]{3}{*}{ Forest management } & $\begin{array}{l}\text { Timely information about crop condition } \\
\text { and phenology }\end{array}$ & $\begin{array}{l}\text { Planning and triggering of farming practices according to specific } \\
\text { phenological stages }\end{array}$ \\
\hline & Water requirements/soil moisture & Irrigation optimisation: only when and where necessary \\
\hline & Final crop productivity & Benefits \\
\hline
\end{tabular}

- Crop type mapping: By means of supervised or partially unsupervised classification schemes, different crop types can be classified in PolSAR images provided that they exhibit different polarimetric signatures as a result of different physical properties.

- Soil moisture retrieval: Using target decomposition approaches, the response from the vegetation and the underlying ground can be separated, as well as the dependence of the radar signal on soil roughness and moisture.

- Phenology monitoring: When plants change their physical characteristics as they develop, different stages can be regarded as different classes, hence being separated by their polarimetric signatures.

Regarding PolInSAR, the rapid rate of growth and change in most crops has undermined so far the development of operational applications based on space-borne SAR systems, since they only provide repeat-pass interferometry (with the notable exception of the recently launched TanDEM-X sensor, which has polarimetric modes available for future use) and agriculture requires single-pass acquisitions. To date, the only successful application based on PolInSAR data is the retrieval of crop height, as an indicator of crop condition or phenology, which has been demonstrated with airborne data.

Wetlands constitute an application scenario with increasing interest in the remote sensing community, especially due to their ecological importance and their clear role in biodiversity. The main applications of PolSAR in wetlands consist in their delineation and characterisation (i.e. condition assessment and vegetation-type classification), for which some approaches have been successfully tested recently. In addition, PolInSAR has been employed to monitor changes in the water level by exploiting the high coherence provided by the double-bounce mechanism produced by the interaction between the water layer and the plants.

Finally, PolSAR has been applied to assess the effect of earthquakes and tsunamis, thanks to the detection of changes in the physical properties of the scene when these events happen.

\subsection{Crop Type Mapping}

\subsubsection{Evaluation of C-Band Polarimetric SAR for Crop Classification}

\subsubsection{Introduction, Motivation and Literature Review}

As the global population increases at a rate of about 80 million per year (United Nations Population Fund 2011), the agriculture and agri-food sector must continue to innovate if it is to meet this demand with an ever-decreasing land base. Recognising the importance of timely information to support accurate national, regional and global agricultural production forecasts, the G20 Group on Earth Observations Global Agricultural Geo-Monitoring Initiative (GEO-GLAM) was launched in 2011 (European Space Agency 2012). Under this initiative, data from Earth observing satellites will be fundamental to characterise crop types and estimate acreages in order to monitor global food production. Yet as the sector adapts to meet the needs of this growing population, society will demand that food be grown in an environmentally sustainable manner. Thus, monitoring how crops are being grown and effects on the landscape and surrounding ecosystems is also important.

Synthetic aperture radars (SARs) can contribute significantly to this global monitoring effort. This is particularly true given improved access in the last decade to satellites with SARs which acquire data at multiple polarisations as well as those that are fully polarimetric-capable. And when agriculture monitoring agencies consider accessing data at multiple frequencies by tasking satellites from various international data providers, a radar-only solution to delivery of crop inventories is possible. Yet research and development are required to adapt existing classification methodologies to use data from SARs. In addition, global cropping systems vary significantly, and consequently, research is required to determine the optimal SAR configuration for different mixes of crops grown under diverse growth cycles. 
In 2004, Agriculture and Agri-Food Canada (AAFC) began developing a classification method to inventory Canada's crops. This research led to the implementation of AAFC's annual crop inventory with the first national inventory available in 2011. Reliability in delivery of the AAFC inventory has been facilitated by acquiring RADARSAT2 dual-polarisation (VV, VH) C-band data to fill gaps when adequate optical data are unavailable (Fig. 3.1). Although the proportion of SAR-to-optical data used in the inventory is growing each year, optical data are still required to meet individual crop accuracy targets. Thus AAFC research continues to explore how multi-frequency and polarimetric SAR data can help to move the inventory towards increased reliance on SAR sensors and less dependence on optical satellites.

SAR satellite data are attractive for mapping and monitoring agriculture as microwave frequencies are unaffected by cloud and haze, unlike their optical counterparts. Agricultural targets (soils and crops) are very dynamic, and thus optical sensors can miss crucial periods in the growing season when information is critically required. Early studies examined the use of single frequency-single polarisation SAR data and reported that these data had to be integrated with optical satellite images if adequate crop classification accuracies were to be achieved. This included integration of $\mathrm{C}-\mathrm{HH}$ (Brisco and Brown 1995), C-VV (Ban 2003; Schotten et al. 1995) or C-HH and C-VV (Blaes et al. 2005) images with at least one optical image. Even when ENVISAT-ASAR and RADARSAT-1 were integrated to create a data set with all three noncoherent linear polarisations $(\mathrm{C}-\mathrm{VV}, \mathrm{C}-\mathrm{VH}$ and C-HH), optical images were still needed to successfully classify individual crops in complex cropping systems (McNairn et al. 2009a). Yet these studies also reported that optically based classification accuracies benefited by the inclusion of SAR data (Brisco and Brown 1995; Ban 2003; McNairn et al. 2009a)

Of all the linear polarisations, many studies have confirmed that the cross-polarisation ( $\mathrm{HV}$ or $\mathrm{VH}$ ) is the single most important polarisation to identify the majority of crops (McNairn et al. 2000, 2009a, b; Foody et al. 1994; Lee et al. 2001). This polarisation is responsive to the multiple scattering from within the vegetation volume, and because

\begin{tabular}{|c|c|c|}
\hline Color & Values & Description \\
\hline & 0 & Unclassified \\
\hline & 20 & WaterWater \\
\hline & 30 & Barren \\
\hline & 34 & Urban \\
\hline & 50 & Shrubland \\
\hline & 80 & Wetland \\
\hline & 110 & Grassland \\
\hline & 122 & Forage Crops \\
\hline & 131 & Fallow \\
\hline & 133 & Barley \\
\hline & 136 & Oats \\
\hline 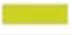 & 137 & Rye \\
\hline & 139 & Triticale \\
\hline & 146 & Wheat \\
\hline & 147 & Com \\
\hline 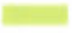 & 153 & Canola/Rapeseed \\
\hline & 154 & Flaxseed \\
\hline & 157 & Sunflowers \\
\hline & 158 & Soybeans \\
\hline 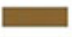 & 162 & Peas \\
\hline 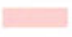 & 177 & Potatoes \\
\hline 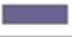 & 179 & Other Vegetables \\
\hline & 181 & Beries \\
\hline$\square$ & 194 & Nursery \\
\hline$\square$ & 220 & Broadleaf \\
\hline
\end{tabular}

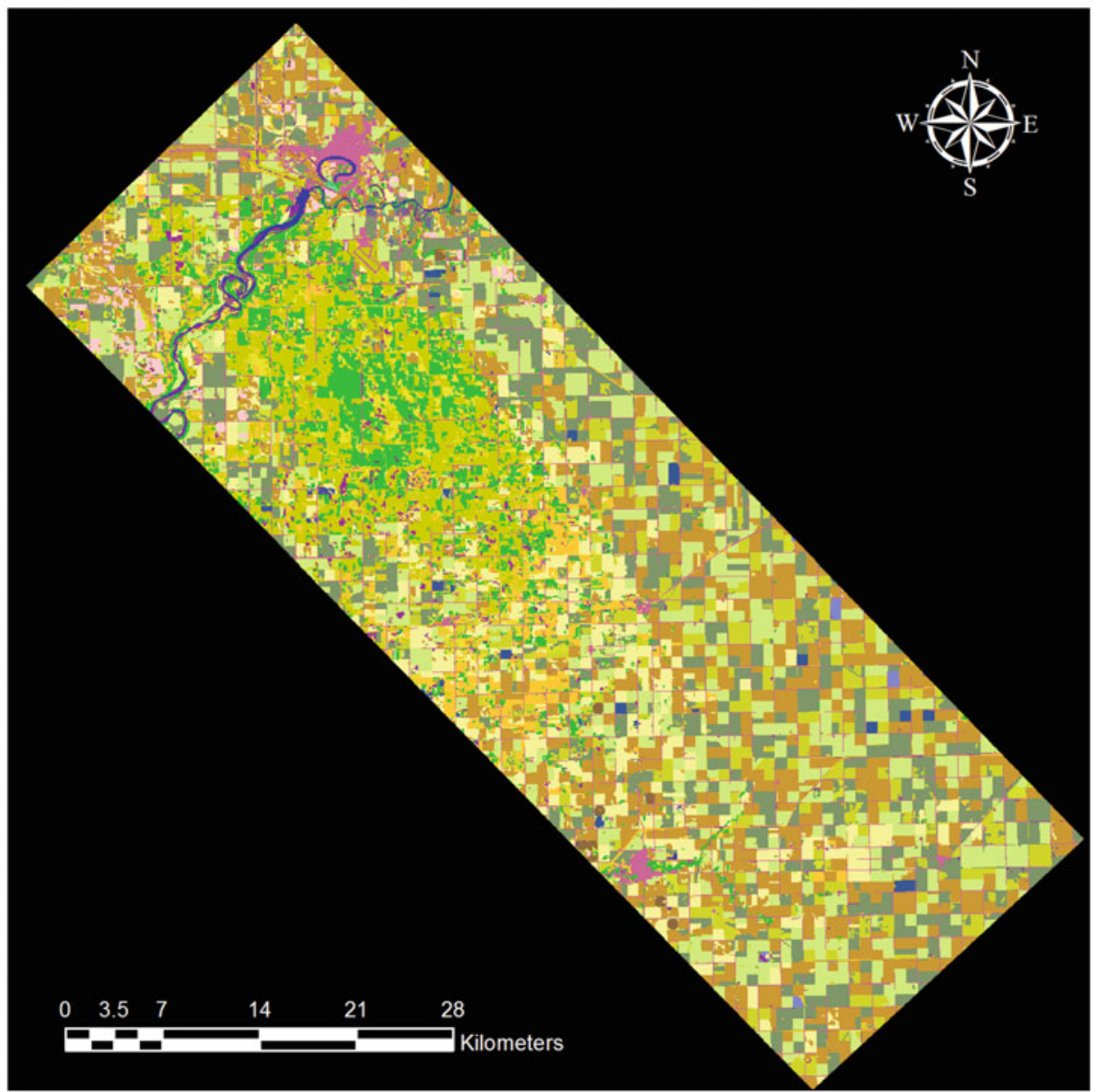

Fig. 3.1 Example of product from Agriculture and Agri-Food Canada's 2012 crop inventory which uses an integration of optical and RADARSAT2 data 
vegetation structure varies greatly among crops, crosspolarised backscatter provides the best discrimination. Nevertheless, classification accuracies are increased substantially with the inclusion of additional polarisations. The greatest incremental increase in accuracy is observed when adding a second polarisation (Foody et al. 1994; McNairn et al. 2000; Hoekman and Vissers 2003), yet a third polarisation will still result in additional improvements to the classification for some crops (McNairn et al. 2000, 2009a; Hoekman and Vissers 2003).

Less research has been completed on the additional benefit of coherent polarimetric parameters for crop classification. Integrating all three linear polarisations and several polarimetric parameters (the co- and cross-polarised pedestal and variation coefficients, e.g.) (Foody et al. 1994) reported a small increase in accuracies. This small incremental increase in accuracy was confirmed in a study where parameters generated from scattering decompositions provided slightly higher accuracies (McNairn et al. 2009b). The HH-VV correlation coefficient and the RR-RL ratio, integrated with $\mathrm{HV}$, have also proven useful in classifying crops (Quegan et al. 2003).

A multi-frequency multi-polarisation approach is the best option for crop discrimination. Ground-based scatterometer and airborne SAR research campaigns describe improvements in vegetation discrimination using multi-frequency data. Researchers reported that relative to single frequency data, higher crop classification accuracies are achieved using $\mathrm{X}$ and C-band (Thomson et al. 1990); C- and L-band (Skriver 2012); X-, C- and L-band (Brisco and Protz 1980; Guindon et al. 1984); as well as C- and L- and P-band (Chen et al. 1996; Ferrazzoli et al. 1997, 1999; Hill et al. 2005; Hoekman et al. 2011) data. The advantage of a multi-frequency data set for separating vegetation types has also been demonstrated using data acquired from multiple satellite platforms, specifically ERS (C-band) and JERS (L-band) (Bouman and Uenk 1992; Dobson et al. 1996). Lower-frequency (i.e. L-band) microwaves penetrate larger biomass crops, and the scattering from within the canopy where structure is quite different aids in separating these crops (McNairn et al. 2009b). Yet for smaller biomass crops, lower frequencies can penetrate too far into the canopy and result in significant soil contributions. For these crops, discrimination is achieved using higherfrequency microwaves where most interaction is limited to within the canopy.

\subsubsection{Methodology}

The evaluation of C-band SAR for crop classification was accomplished using RADARSAT-2 data acquired for three study sites - two in Canada's western Prairie region and a third in eastern Canada. Field observations of crop type were collected to train the supervised classification model and to test the classification accuracy. Fields were randomly split with half used for training and the remainder used to independently evaluate the classification results. A $7 \times 7$ boxcar speckle filter was applied to the single-look complex RADARSAT- 2 data for the eastern site. For the two western sites, a $9 \times 9$ boxcar filter was selected due to the larger field sizes typical of this region of Canada. The covariance matrix was then converted to a symmetrised 3 by 3 covariance matrix from which radar intensity backscatter $(\mathrm{HH}$, HV/VH, VV) was generated. In addition, the Cloude-Pottier (Cloude and Pottier 1997) decomposition was applied generating entropy $(H)$, anisotropy $(A)$ and alpha angle $(\bar{\alpha})$ parameters. With the Freeman-Durden decomposition, the total power was partitioned into contributions from three scattering mechanisms: single-bounce, double-bounce and volume scattering (Freeman and Durden 1998). After generating these radar parameters, the data were orthorectified using platform ephemeris information and models of the internal sensor distortion, a set of ground control points (GCPs) and digital elevation models. Prior to classification an enhanced Lee filter was applied with a window size of $5 \times 5$ for the eastern site and $9 \times 9$ for the western sites.

Fully polarimetric sensors are limited in terms of swath coverage, making their use in large area monitoring a challenge. Specific to RADARSAT-2 the maximum swath for quad-pol (QP) is approximately $50 \mathrm{~km}$, achieved with the wide QP mode. With compact polarimetric (CP) configurations, a larger swath is maintained relative to fully polarimetric SAR systems (Charbonneau et al. 2010), making CP an attractive option to assist with the production of national crop inventories. Although CP offers this operational advantage, the information generated by $\mathrm{CP}$ configurations is not well understood particularly as it relates to applications such as crop classification. To investigate the potential of CP for this application, a circular right transmitting-coherent linear receiving compact polarimetric mode (CL-pol) was simulated from the RADARSAT-2 fully polarimetric data acquired for the Casselman site. The simulated CL-pol data were stored in the Stokes vector format, and the Stokes vector parameters were subsequently used in the classification. A detailed description of the CP simulation in provided in (Charbonneau et al. 2010).

Upon evaluation of several classification models (McNairn et al. 2009a), AAFC chose a decision tree (DT) supervised classifier as it provided the most consistent and reliable classification accuracies particularly when SAR data were used in the classification. Decision boundaries and coefficients for the linear discriminate function used in the classification were estimated empirically from the training data. The DT was run using boosting over five trials to force the classifier to focus on poorly classified cases. To avoid over-fitting, the classifier used a global pruning of the model of $25 \%$. The DT classifier was implemented with the software See5 (Rulequest Research 2008) and is currently the basis of AAFC's operational annual crop inventory. 


\subsubsection{Experimental Results}

Test sites and corresponding radar and validation data sets selected for crop classification are summarised in Table 3.2 and further described in the Appendix.

When a multitemporal data set with all linear polarisations $(\mathrm{HH}, \mathrm{VV}, \mathrm{HV} / \mathrm{VH})$ is available, high overall accuracies are achievable. With the simple crop mix (corn, soybeans, wheat, pasture) of the eastern site, accuracies of $90 \%$ are reached. Slightly lower accuracies are found in more complex cropping systems, such as those of Canada's western region. Here overall accuracies of $85.5 \%$ are reported for both the Manitoba and Saskatchewan sites. Using either the CloudePottier (western sites) or Freeman-Durden (all three sites) decomposition parameters resulted in higher accuracies although gains were small. A classification using CloudePottier entropy, alpha and anisotropy inputs increased overall accuracies by less than a percentage for the Manitoba site $(86.3 \%)$ and less than $2 \%$ for the Saskatchewan site (87.3\%). With the simple cropping mix in the eastern site, the linear polarisations outperformed the accuracies achieved with Cloude-Pottier (87.5\%). The scattering parameters generated by the Freeman-Durden decomposition provided the best results for all three sites (Ontario, 91.3\%; Manitoba, 87.1\%; Saskatchewan, $88.7 \%$ ) although increases in accuracy were limited to between 1 and 3\%. For the Casselman site, the CL-pol Stokes vector parameters produced an overall accuracy $(91.1 \%)$ similar to that achieved with the FreemanDurden decomposition (91.3\%).

Although high overall accuracies are an important indicator of a successful classification, crops must also be well classified individually in order to provide accurate production estimates. Reaching these accuracies has been a challenge for SAR-only classifications. For the Canadian inventory, achieving these individual crop accuracies has required integration of optical data particularly where the cropping mix is more complex. As presented in Fig. 3.2, when the three linear polarisations achieve high crop-specific accuracies (greater than $90 \%$ ), little is gained in using fully polarimetric or $\mathrm{CP}$ parameters. However, the Cloude-Pottier or Freeman-Durden decompositions can improve accuracies when the linear polarisations are unable to reach accuracies above $90 \%$. The results from the decompositions $(\mathrm{QP})$ and the Stokes vector parameters $(\mathrm{CP})$ were comparable with differences of less than one percent observed. The one exception was the pasture class, where the Freeman-Durden outperformed the Stokes vectors by about $3 \%$. It should be noted that small incremental increases in accuracies are important as these improved accuracies lead to more precise acreage estimates when considering the generation of national, regional or global production assessments.

\subsubsection{Comparison with Single-/ Dual-Polarisation Data}

As expected, the use of a single polarisation does not achieve satisfactory classification results. For the more complex cropping mix found in Carman, single VV and VH polarisations produced overall accuracies of $75.6 \%$ and $75.1 \%$, respectively. Slightly higher accuracies were reached in the eastern site (77.9\% for VV polarisation and $79.4 \%$ for $\mathrm{VH})$. Only 62.5\% (Casselman) and 68.1\% (Carman) were attained with the HH polarisation. For the western cropping mix, while the VV polarisation provided better classification of pasture, corn and cereal crops, the remaining crops (canola, flax and beans) were best identified with the cross-polarisation. In Ontario, VH outperformed VV for all crops except corn. For both sites, $\mathrm{HH}$ always produced the lowest crop-specific accuracies.

As previously reported, the addition of a second polarisation (in this case adding $\mathrm{VV}$ to $\mathrm{VH}$ ) provided significant improvements for many crops. For a more complex cropping mix (Carman), with these two polarisations, accuracies for corn, beans and cereals increased by $17.8 \%$, $13.5 \%$ and $8.0 \%$, respectively (Fig. 3.3). The addition of $\mathrm{HH}$ to these first two polarisations increased accuracies by less than $2 \%$. In the eastern site, the integration of $\mathrm{VV}$ with $\mathrm{VH}$ increased individual accuracies by $7-8 \%$ for all crops except soybeans. For soybeans this increase was limited to less than $2 \%$. For the Casselman site, there was value in adding the third $\mathrm{HH}$ polarisation for pasture and wheat where accuracies rose by a further $10-11 \%$ (Fig. 3.3).

In regions of Western Canada, approximately threequarters of the annual cropland is planted in cereals and canola. In the eastern part of the country, this is true for soybeans and corn. Early estimates of these specific crop acreages are important for production and yield forecasting.

Table 3.2 Test sites and corresponding radar and validation data selected for the generation of showcases on crop classification

\begin{tabular}{|c|c|c|}
\hline Application/product & Test site - radar data & Reference data \\
\hline \multirow[t]{7}{*}{ Crop classification } & Casselman, Ontario (2008) & \multirow[t]{2}{*}{274 fields surveyed for crop type } \\
\hline & RADARSAT-2 FQ19 June 22, July 16, Aug. 9, Sept. 2 & \\
\hline & Carman, Manitoba (2009) & \multirow[t]{2}{*}{219 fields surveyed for crop type } \\
\hline & RADARSAT-2 FQ15 June 22, July 16, Aug. 9, Sept. 2 & \\
\hline & Indian Head, Saskatchewan ('09) & \multirow[t]{3}{*}{610 fields surveyed for crop type } \\
\hline & RADARSAT-2 FQ2 June 8, July 2, July 26, Aug. 19, Sept. 12 & \\
\hline & RADARSAT-2 FQ19 June 4, July 22, Aug. 15, Sept. 8 & \\
\hline
\end{tabular}



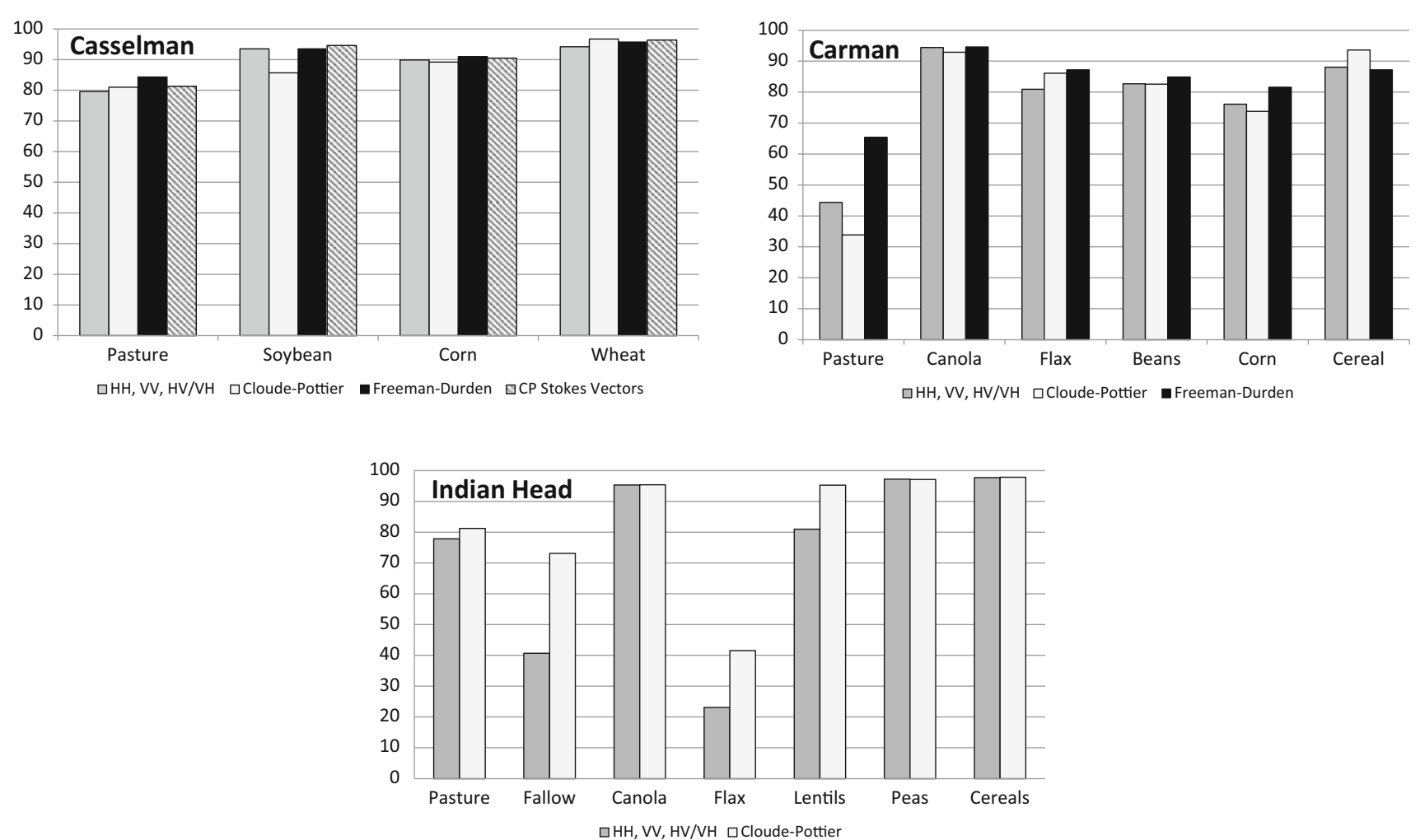

Fig. 3.2 Producer's accuracies for the three study sites
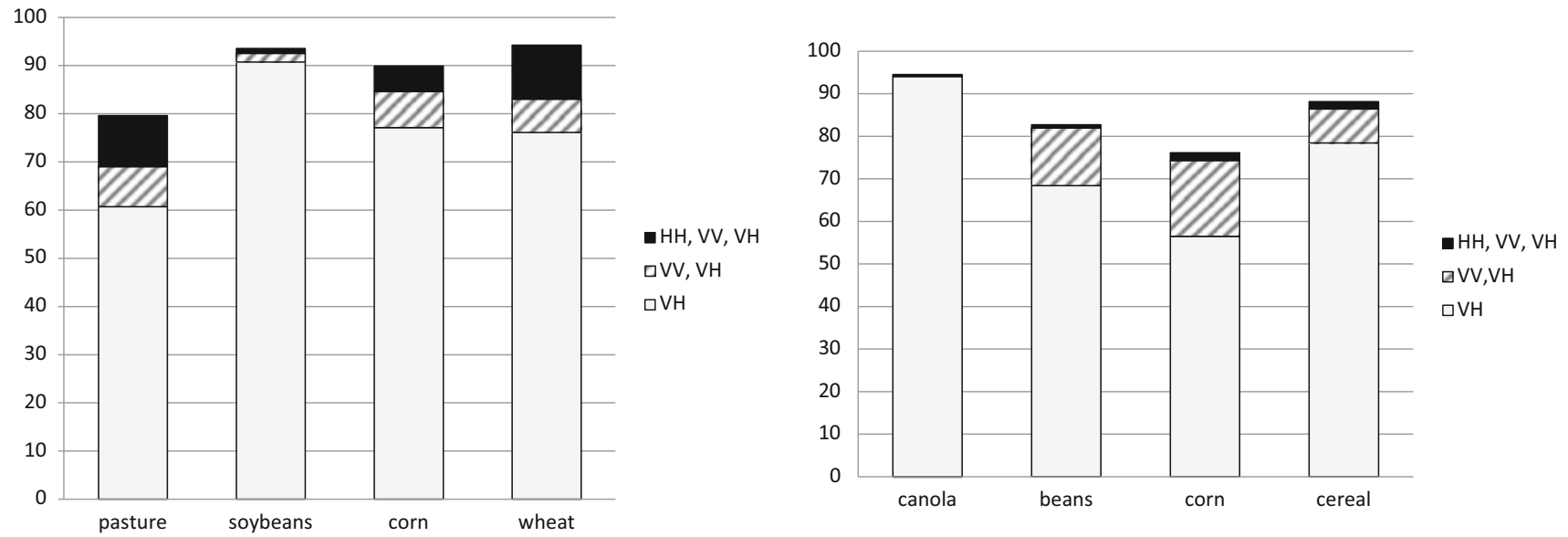

Fig. 3.3 Comparison of crop-specific producer's accuracies using single, dual and multiple polarisations for Casselman (left) and Carman (right)

By mid-July, using only two RADARSAT-2 images (June 22 and July 16), accuracies above 70\% (canola) and 80\% (cereals) could be reached if either the Cloude-Pottier or Freeman-Durden decompositions were used in the classification of the western site. This represents a significant improvement in accuracies reached relative to those generated using single $(\mathrm{VH})$, dual $(\mathrm{VV}, \mathrm{VH})$ or multiple $(\mathrm{HH}, \mathrm{VV}, \mathrm{VH})$ polarisations (Fig. 3.4). For the eastern Ontario site, mid-season estimates (as well as by July 16) were significantly improved using the $\mathrm{QP}$ or $\mathrm{CP}$ configurations. Using either the simulated CL-pol data or one of the decompositions, accuracies close to $80 \%$ were achieved for corn and $93 \%$ for wheat. The Stokes vector parameters were far superior for mid-season identification of soybeans (79.9\%), about $10 \%$ better than accuracies reached with either of the decompositions and $6 \%$ better than accuracies produced using the three linear polarisations. 

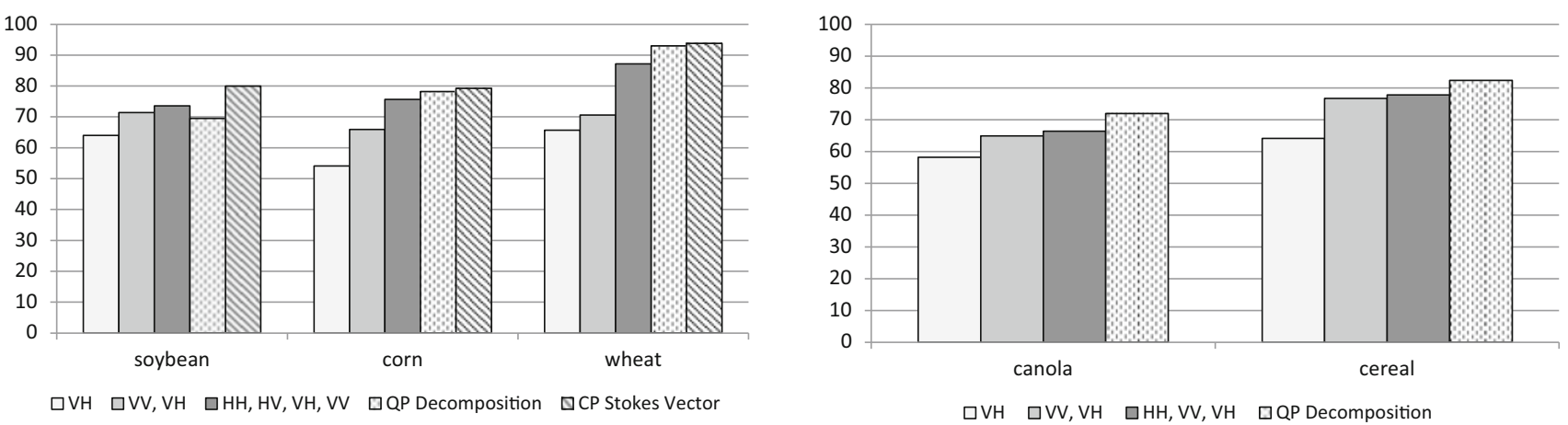

Fig. 3.4 Mid-season classification accuracies for Casselman (left) and Carman (right)

\subsubsection{Discussion on the Role of Polarimetry, on the Maturity of the Application and Conclusions}

Accurate crop classification is the basis for generating production estimates and yield forecasts. Regardless of the source of satellite data, multitemporal acquisitions are necessary. These map products have traditionally been generated using optical satellite data. However, with improved access to SAR satellites with greater polarisation diversity, and in some cases polarimetric capabilities, the potential of this technology for agriculture monitoring is evident. The use of SAR for crop classification requires multitemporal acquisitions of dual polarisations (at a minimum) to meet acceptable overall classification accuracies. However, cropspecific accuracies still require the integration of additional data which has often come from optical sensors. Yet with increased access to polarimetric satellites, results are demonstrating that parameters generated from these complex data, such as radar decompositions, can improve cropspecific identification. In particular, fully and compact polarimetric data can assist in crop identification mid-season which is important if production estimates are required before end of season.

Repetition in results by many international researchers has brought confidence to the idea of radar-assisted crop identification. This has been put into practice by Agriculture and Agri-Food Canada where RADARSAT-2 (VV, VH) data are integrated with optical imagery to produce that country's annual inventory. Increasing the ratio of radar-to-optical data in such an inventory is now limited primarily by access to polarimetric swaths deemed to be operationally appropriate for national mapping. Missions which include wide swath compact polarimetry modes may provide a partial solution. Further, researchers have repeatedly demonstrated that a radar-only approach, with no optical data requirement, is possible with the integration of multi-frequency SAR. The primary impediment to implementing this approach is access to multi-frequency data over swaths appropriate for large area mapping. Without a single multi-frequency satellite, coordinating access from multiple sensors (from multiple agencies) is required. If this challenge can be met, monitoring agencies would have the classification tools and data to monitor national, regional and global agriculture production exclusively with SAR sensors.

\subsubsection{Crop Classification Using Multitemporal L- and C-Band Airborne Polarimetric SAR}

\subsubsection{Introduction, Motivation and Literature Review}

Remote sensing has the potential to provide estimates of inputs for land process models, for instance, the land cover type as well as quantitative geophysical parameters such as soil moisture and leaf area index (LAI). Such models can provide information that is crucial for a number of applications such as flood and drought prediction, crop irrigation scheduling and meteorology (Moran et al. 2004). Also, land cover maps provide fundamental information for many aspects of land use planning and policy development (Bocco et al. 2001), as a prerequisite for monitoring and modelling land use and environmental change (Shalaby and Tateishi 2007) and as a basis for land use statistics at all levels. The European Environmental Agency (EEA) has produced the CORINE Land Cover, and the requirement is a classification accuracy of $85 \%$ for the classification performance of the so-called level 3 classes, which are relatively broad classes (EEA 2007). An important part of land cover is the distribution of agricultural crops, and it is well-known that synthetic aperture radar (SAR) is able to perform such classification. The main advantage of SAR compared with optical and infrared sensors is its all-weather mapping capability. The discrimination potential of SAR data is based on the sensitivity of the radar backscatter to dielectric properties of the objects and to object structure (i.e. the size, shape and orientation distribution of the scatterers) (Skriver et al. 1999). The possibility of identifying individual classes is based on the fact that the dielectric properties and structure of different 
crop types vary. A distinct variation is also seen for these properties through the growing season due to the development of crops (Skriver et al. 1999). Therefore, the discrimination capabilities may vary through the year, and it may also be improved by performing multitemporal classification (Schotten et al. 1995; Skriver 2012; Skriver et al. 2011). The radar backscatter is also sensitive to other parameters, e.g. dielectric properties of the soil, surface roughness, terrain slope and vegetation canopy structure (e.g. row direction and spacing and cover fraction) (Moran et al. 2004). These properties are not necessarily specific for the individual classes and may therefore cause variability of the backscatter and other polarimetric observables within the classes. Also, differences in the development stages at a specific point in time due to, for instance, differences in sowing time may cause such variability (Skriver et al. 1999).

Polarimetric SAR measures all polarimetric information for a target in the form of the scattering matrix. Therefore, such data may be used to assess the capabilities of SAR for crop classification in various configurations, including single-polarisation, dual-polarisation and fully polarimetric modes. Various methods have been used for such assessments: statistical methods based on the Wishart distribution (Lee et al. 1994; 2001; Skriver 2012; Skriver et al. 2011) or covariance matrix elements transformed into backscatter coefficients (Lee et al. 2001; McNairn et al. 2009b; Hoekman and Vissers 2003; Skriver 2012; Skriver et al. 2011; Foody 1988; Freeman et al. 1994), methods based on scattering mechanisms (Lee et al. 1999, 2004) and knowledge-based methods (Ferrazzoli et al. 1999; Pierce et al. 1994; Skriver 2001). In the latter approaches, it is possible to include scattering model results and common knowledge about the targets. Hence, such methods are normally relatively robust and easy to adjust to different growing conditions caused by, for instance, different sowing time, soil and weather conditions. The number of different classes that can be determined is normally relatively small using this type of method. The statistically based methods, on the other hand, will normally provide a larger number of classes, but the classifiers will then normally be specifically adjusted to the data set at hand, and it is difficult to adapt the classifier to other environmental conditions and/or geographical locations.

\subsubsection{Methodology}

The basis for our analysis and processing is the multi-look covariance matrix, which is formed from the coherent scattering vector made up of the backscatter responses at the linear polarisations, $\mathrm{HH}, \mathrm{VV}$ and cross-polarisation. The diagonal backscatter elements in the covariance matrix are then normalised so they correspond to the $\gamma^{0}$ backscatter coefficient in order to reduce the influence of the incidence angle.
All acquisitions have been co-registered by identifying ground control points in the images. The covariance matrix data, corresponding to the original one-look scattering matrix data, have been averaged to reduce the speckle by a cosinesquared weighted 9 by 9 filter. After averaging the equivalent number of looks is estimated to be 9-11 from homogenous areas in the images in the Foulum data set and 10 in the AgriSAR06 data set.

Three different SAR modes are simulated using the same data set, i.e. single-polarisation, dual-polarisation and fully polarimetric data. For the polarimetric results, the full covariance matrix data has been used. For the single- and dual-polarisation modes, subsets of the covariance matrix data have been used, e.g. for the single-polarisation backscatter coefficients, $\gamma_{h h}^{0}$ and $\gamma_{v v}^{0}$, the corresponding elements from the diagonal of the covariance matrix have been used, and for the dual-polarisation backscatter coefficients, pairs of these elements have been used, as well as the cross-polarised backscatter, $\gamma_{x p}^{0}$. For the single- and dual-pol modes, the backscatter intensities have been used, and hence not the complex scattering matrix elements, in order to simulate satellite systems, like ENVISAT, RADARSAT, ALOS and Sentinel-1, where such modes are used to optimise the spatial and temporal coverage. Hence, the possibility of a coherent combination of the $\mathrm{HH}$ and VV polarisations has not been considered for this application.

For the single- and dual-polarisation data, the backscatter coefficients have been used in the classification. Backscatter coefficients follow the Gamma pdf for a multi-look SAR image. When the number of looks is large, the Gamma pdf can, however, be approximated by the Gaussian pdf. The number of looks in our case is at least 10 . Hence, the Gaussian pdf is a valid approximation, and the multivariate Gaussian pdf is used for both single- and dual-polarisation data. The classification method used in all cases is the standard Bayesian maximum likelihood (ML) classifier for multivariate Gaussian statistics.

For the fully polarimetric cases, two methods are used: the standard Bayesian maximum likelihood Wishart classifier originally proposed by Lee et al. (1994) and the method introduced by Hoekman and Vissers using a reversible transform of the covariance matrix into backscatter intensities (Hoekman and Vissers 2003). The complex Wishart classifier is based on the classical Bayesian approach to classification, and the distance measure is derived based on the pdf of the covariance (or coherency) matrix for polarimetric SAR data, i.e. the complex Wishart pdf (Lee et al. 1994). Hoekman and Vissers (2003) introduced a reversible transform of the covariance matrix into backscatter intensities. The advantage is that the fully polarimetric information can be described by backscatter intensities alone. This description will, for instance, better than the Wishart distribution, describe the statistics of a collection of homogeneous areas for the same 
class but with some variability of the mean parameters due to, e.g. slightly different development stages for crops.

The methodology for the assessment of the classification accuracy that has been used is the following: one relatively large and representative field (more than 500 pixels) has been picked for each of the crop classes, and these areas have been used as training areas for the classifiers. A large number of test areas for the classes have been selected, and the classification accuracy has been estimated using these areas. The classification errors estimated are based on the number of pixels in all these test areas, and hence no results are shown using the same data set for training and for testing.

\subsubsection{Experimental Results}

Test sites and corresponding radar and validation data sets selected for crop classification are summarised in Table 3.3 and further described in the Appendix.

The results of classifying the crops for different modes of the SAR acquisitions and for the multitemporal configurations are shown in Figs. 3.5 and 3.6. For each frequency and test site, all single-polarisation and incoherent dual-polarisation configurations have been assessed, as well as the two polarimetric classification methods when fully polarimetric data were available. In the two figures, only the configuration with the best performance, i.e. the lowest classification error, within these groups of configurations is shown. For the Foulum data set, it is seen in Fig. 3.5 that the polarimetric data always provide the best classification result, especially at L-band, where there is a relatively large improvement compared to the incoherent dual-polarisation results. At C-band, the difference between polarimetric results and the dual-polarisation results is smaller. At both frequencies, the Hoekman and Vissers classifier performs best, and the classification error is down to approximately $20 \%$. Also at both frequencies, a clear improvement is seen using the multitemporal acquisitions.

At the AgriSAR06 test site, polarimetric data were available only at L-band, and in this case, as can be seen from Fig. 3.6, there is a clear advantage when only a few temporal acquisitions are available, whereas the performance is worse than the incoherent dual polarisation for more acquisitions. The best classification result is about $11 \%$.

\subsubsection{Comparison with Single-/ Dual-Polarisation Data}

As mentioned above, also single- and incoherent dualpolarisation results are shown in Figs. 3.5 and 3.6. For both test sites at C-band, it is seen that the best single polarisation is $\mathrm{VV}$ and the best dual polarisation is VVXP, whereas at L-band the best single polarisation is $\mathrm{HH}$ and the best dual polarisation is HHXP.

Table 3.3 Test sites and corresponding radar and validation data selected for the generation of showcases on crop classification

\begin{tabular}{l|l|l}
\hline Application/product & Test site - radar data & Reference data \\
\hline Crop classification & Foulum & Crop maps \\
\hline & C- and L-band fully polarimetric multitemporal data \\
\hline & AgriSAR06 & \\
\hline & C-band dual pol., multitemporal \\
\hline & L-band fully pol., multitemporal & \\
\hline
\end{tabular}

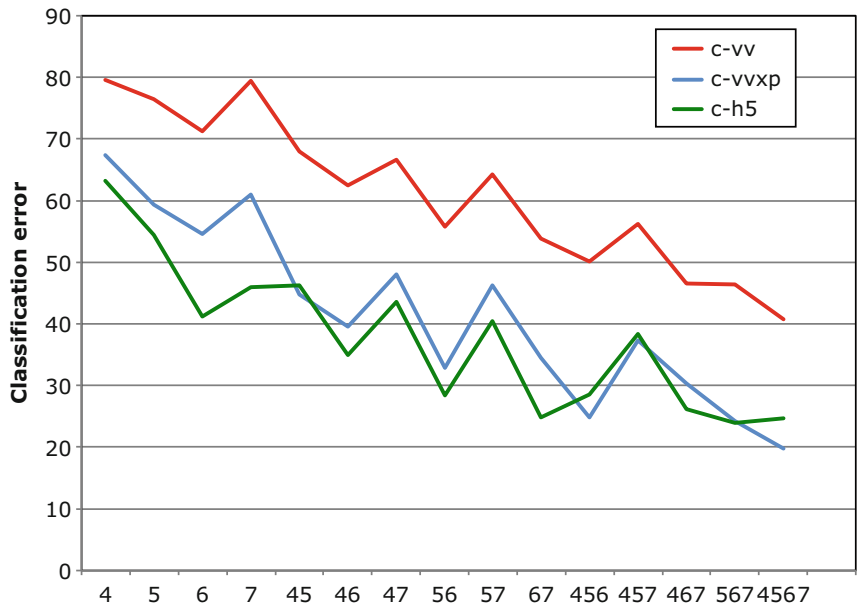

Fig. 3.5 Classification errors for Foulum at C-band (left) and L-band (right) for the best modes for the three cases, single polarisation (red), dual polarisation (blue) and full polarimetry (green), as a function of the

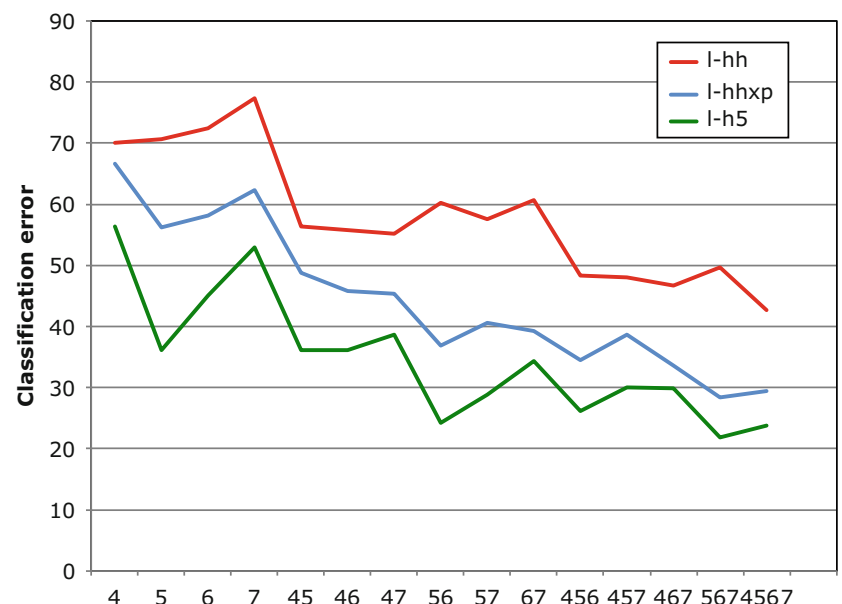

multitemporal acquisition mode, where 4, 5, 6 and 7 correspond to April, May, June and July, respectively 


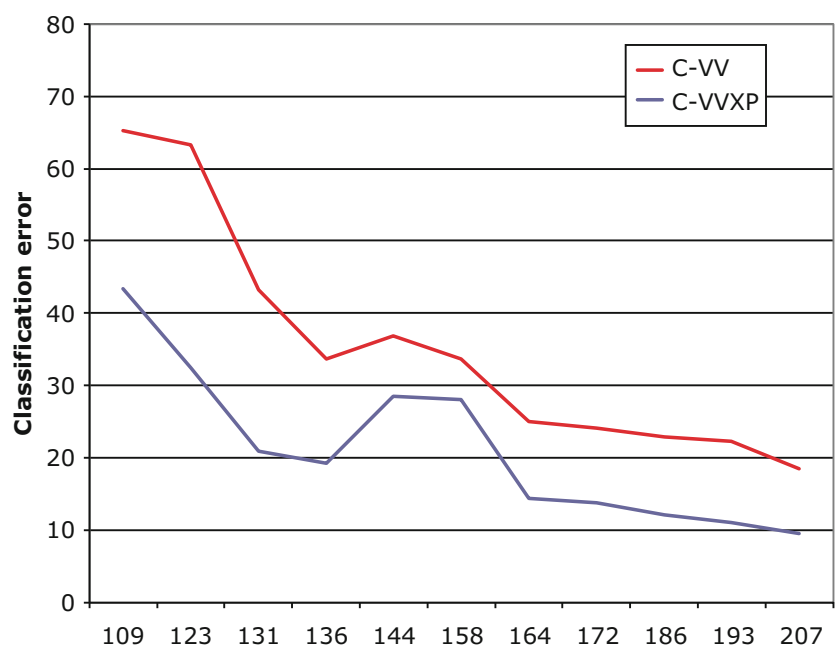

Fig. 3.6 Classification errors for AgriSAR06 at C-band (left) and L-band (right) for the best modes for the three cases, single polarisation (red), dual polarisation (blue) and full polarimetry (green) (only

For the Foulum test site, the smallest classification error for the C-band results is $41 \%$ for VV and $20 \%$ for VVXP, and for the L-band results, it is $43 \%$ for $\mathrm{HH}$ and $28 \%$ for HHXP (Skriver 2012). For the AgriSAR06 test site, the results are at C-band $19 \%$ for VV and $10 \%$ for VVXP, and at L-band $15 \%$ for $\mathrm{HH}$ and 3\% for HHXP (Skriver et al. 2011). It is observed that the differences in performance between the C- and the L-band configurations are relatively small. For the Foulum test site, the C-band performs better than L-band, whereas it is the other way around for the AgriSAR06 test site. This probably has to do with the different set of crop types present at the two test sites.

In all cases, there is a large improvement from the singlepolarisation to the dual-polarisation results. It is clearly seen that the multitemporal acquisitions are necessary for these configurations in order to obtain a reasonable performance. Especially, the dual-polarisation modes including the crosspolarisation perform well, and at C-band they provide comparable results with the fully polarimetric data.

\subsubsection{Discussion on the Role of Polarimetry on the Maturity of the Application and Conclusions}

From the presented results, it is clear that polarimetry has a role in crop classification from SAR. Especially, for L-band an improvement is seen compared to the single and incoherent dual polarisations. At C-band, the polarimetric SAR still performs best, but the improvement compared to the simpler modes is small. The classification results for C-and L-band are relatively similar, and there is no clear preference for one or the other, except that L-band probably requires the polarimetric modes to obtain a sufficient classification performance, whereas simpler modes may be sufficient at C-band.

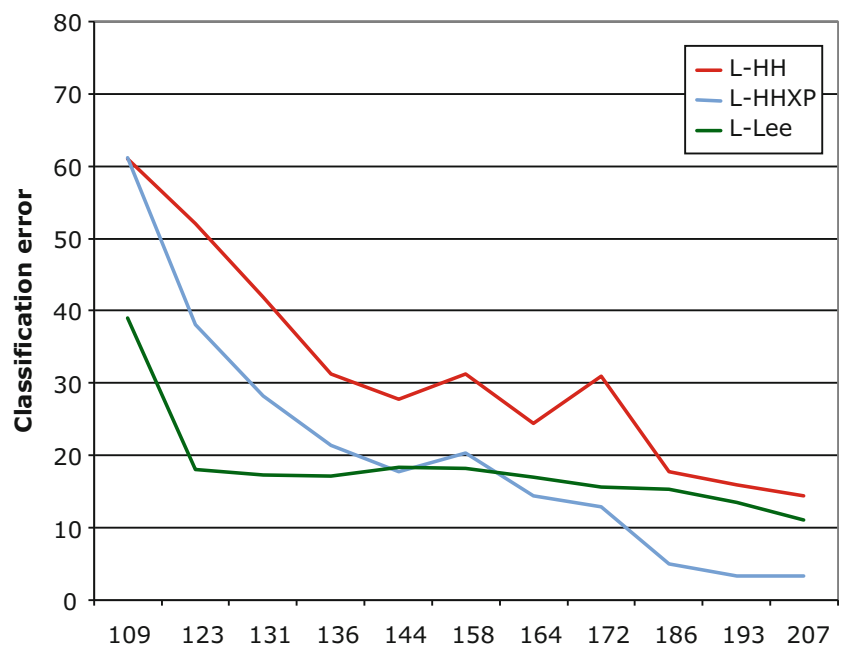

L-band), as a function of the multitemporal series, where the day number corresponds to a series of acquisitions up to and including that day

The application of using multitemporal SAR for crop classification is considered to be relatively mature. Multitemporal acquisitions are considered to be the most important requirement for this application, whether polarimetric or simpler modes are available or not. For the polarimetric modes, it may be less critical if a lot of multitemporal acquisitions are not available, whereas it is essential with multitemporal acquisitions for the single- and dual-polarisation modes.

In conclusion, the performance of the multitemporal SAR data is seen to be very close and even better for some of the modes, compared with the requirements on, e.g. the CORINE land cover of $85 \%$ classification accuracy on level 3 classes. Hence, it will be possible with future polarimetric and/or short revisit systems to obtain a sufficient classification performance. This could, for instance, be possible with the future Sentinel-1 satellites, where single- and dualpolarisation acquisitions at C-band will be available with short revisit times for large parts of the continents, or with future L-band polarimetric missions, such as ALOS-2.

\subsection{Soil Moisture Estimation Under Vegetation Using SAR Polarimetry}

\subsubsection{Introduction, Motivation and Literature Review}

Several hydrological processes, like surface runoff, infiltration, plant water uptake and groundwater drainage, are affected by the spatial and temporal variations of soil moisture within the pedosphere (Bronstert et al. 2012; Krajewski et al. 2006). These moisture changes are still very difficult to 
simulate by environmental/hydrological models due to the multiple and multi-dimensional dependencies of this highly varying soil parameter. Therefore they require a continuous and long-term monitoring (Meehl et al. 2007).

Monitoring of soil moisture content reveals, for instance, the retention capabilities of the soil to store the infiltrated precipitation for a delayed and uncritical release in the discharge system (Bronstert and Bardossy 1999). In this way hazard precautions, like flood forecasting and dam management, can be supported to identify critical catchment states before flooding events (Minet et al. 2011). Besides flood prevention in humid regions, also agricultural management practices in semi-arid regions, like irrigation, benefit from the incorporation of soil moisture information leading to crop yield optimisation in terms of precision farming (Robinson et al. 2008).

In practice, information about the soil moisture status is mostly acquired by single point measurements (Robinson et al. 2003), which might be combined to wireless sensor networks in order to enlarge the monitoring to field scale (Cardell-Oliver et al. 2005). In addition, soil moisture sensing on this scale can be also approached by geophysical methods, like ground-penetrating radar (GPR) (Huisman et al. 2003) or ground albedo neutron sensing (GANS) with cosmic ray probes (Zreda et al. 2008). As the spatial upscaling from point or field measurements to large scales is highly non-linear (Merz and Plate 1997), remote sensing extends soil information retrieval from local point or field studies to spatial investigations on sub-catchment or entire catchment areas, including also very isolated regions (Western et al. 2002).

Since optical remote sensing of soil properties (Katra et al. 2006) relies on daylight and clear weather conditions, RADAR-based methods turn out to be weather- and illumination-independent supporting a continuous soil monitoring strategy. Therefore passive microwave sensors (radiometers) (Jackson 1993) as well as active microwave sensors (scatterometers (Wagner et al. 2007), synthetic aperture radars (SAR) (Ulaby et al. 1982)) are used on airborne or space-borne platforms.

Space-borne radiometers and scatterometers, like MIRAS on SMOS (Kerr et al. 2001) and AMI on ERS, operate with low spatial resolution in the order of kilometres and high temporal resolution in the range of one acquisition every second or third day (in mid-latitudes), which is favourable for global monitoring purposes.

In contrary, SAR sensors, like PALSAR on ALOS (Rosenqvist et al. 2007) or ASAR on ENVISAT, acquired data in the order of decametres with a temporal resolution in the range of one acquisition every first to second week (in mid-latitudes), which fits to regional imaging purposes (Wagner et al. 2008). In order to combine both spatial scales for a spatially enhanced soil moisture retrieval on a global scale, an active-passive microwave sensor will be launched for the SMAP mission (Entekabi et al. 2010).

However, only SAR remote sensing enables a monitoring of the soil and its variability down to the local scale due to its imaging capabilities in high spatial resolution compared to the other microwave sensors. The sensitivity of SAR for soil characteristics is based on the fact that the transmitted electromagnetic (EM) wave interacts with two key parameters of the soil system: soil moisture and soil roughness. SAR sensors, used for soil moisture retrieval, acquire in different wavelengths (ranging from X-band $(\lambda=3 \mathrm{~cm}$, TerraSAR-X (Anguela et al. 2010)), C-band ( $\lambda=5 \mathrm{~cm}$, ENVISAT-ASAR (Löw et al. 2006)), L-band ( $\lambda=23 \mathrm{~cm}$, ALOS (Koyama and Schneider 2011)) to P-band ( $\lambda=80 \mathrm{~cm}$, E-SAR (Jagdhuber et al. 2012a)).

Moreover, SAR remote sensing has the capability to penetrate through natural media, like vegetation layers, especially with increasing wavelength (Cloude et al. 2004). Hence, also agricultural regions, which are most of the year covered by vegetation, can be investigated for their soil moisture conditions. This enables a continuous soil monitoring along the entire plant growth cycle, if the increasing scattering contribution from the vegetation is considered (Hajnsek et al. 2009).

Concerning SAR remote sensing approaches, the first soil moisture analyses were carried out on single-polarisation intensities over bare soils leading to ill-posed inversion problems, because the soil moisture and roughness influences are enclosed in one acquired signal (Bernard et al. 1982). With the emerging establishment of fully polarimetric SAR sensors, the observation space has been enlarged allowing to separate soil roughness from soil moisture influence for an unambiguous inversion of both soil parameters over bare soils (Hajnsek et al. 2003).

For bare soils, three different categories of EM scattering models, which relate the natural soil conditions with the characteristics of the scattered RADAR signature, can be distinguished: empirical models, semi-empirical models and theoretical models. As remote sensing relies on the understanding of electromagnetic (EM) scattering on ground, only theoretical models can be considered as test site independent and form the basis of the analyses. Among these models, the integral equation method (IEM) is widely applied for EM scattering of bare soils because of its broad range of applicability in terms of soil roughness classes (Fung 1994).

So far the investigation of soil parameters, like soil moisture, with polarimetric SAR remote sensing was constrained on bare soil areas due to the lack of retrieval algorithms including the influence of a vegetation cover (Hajnsek et al. 2003). The dielectric discontinuities in vegetation volumes, which can be, for example, stems, branches, twigs and leaves, scatter the waves in a complex manner (Ulaby and Wilson 1985). 
In order to incorporate the influence of vegetation cover, different EM models have been developed to describe vegetation scattering (Lang and Sidhu 1983), whereby the level of detail varies from a simple layer of uniformly shaped spheres (Attema and Ulaby 1978) until sophisticated threedimensional models of the vegetation volume, including cylinders, dipoles and discs with their respective orientation distributions to simulate stems, branches and leaves (Karam et al. 1992). Especially for the latter models, the amount of required input parameters exceeds by far the potential observable space of SAR remote sensing for direct inversion.

Unlike traditional retrieval approaches evaluating the total backscattered signature, an innovative soil information retrieval under vegetation cover should focus on the separation of the individual scattering contributions from the soil and the vegetation in order to extract only the soil properties for inversion. Polarimetric decompositions represent an established technique for separation of elementary scattering processes and have quite a history in polarimetric SAR remote sensing. Originally, these decomposition methods were designed for forested environments including scattering interactions with the ground, with the tree trunk and the ground as well as with the tree canopy. Recently, the description of the polarimetric vegetation volume component was considerably extended by Freeman (Freeman 2007), Neumann (Neumann et al. 2010) and Arii (Arii et al. 2010) using shape parameters for the scattering components (leaves, twigs, branches) and different orientation distributions within the tree canopy to characterise the polarimetric volume scattering of various tree species in a more detailed way. Besides the polarimetric decomposition models for forests, several (multi-angular) polarimetric decomposition methods for the more complex scattering scenario of agriculture, including a depolarising surface component due to soil roughness or an oriented vegetation component due to plant structure, are under development and provide the methodical foundation for this application showcase (Hajnsek et al. 2009; Jagdhuber et al. 2012b, 2013a).

\subsubsection{Methodology}

The retrieval of soil characteristics by polarimetric SAR remote sensing is investigated for agricultural areas using C-band wavelength $(\lambda=5 \mathrm{~cm})$ to assess a continuous soil parameter estimation with a growing vegetation cover along the phenological cycle. Due to polarimetric decomposition techniques, the separation of vegetation from soil/ground information should allow a continuous monitoring of soil moisture under vegetation cover. A modular polarimetric decomposition for retrieval of soil moisture is introduced to provide a profound basis for representation of different natural scattering conditions.

The received SAR signal of almost any natural media is a mixed response of different scattering processes occurring within the resolution cell. Therefore polarimetric decomposition techniques have been developed and used to separate the different scattering contributions. Equation (3.1) describes a generic example of an incoherent decomposition using the coherency matrix notation (Cloude 2009). A simple and generic case of model-based decompositions considers a non-penetrable surface superimposed by a volume of particles. This can be modelled with three components defined as surface $\mathbf{T}_{S}$, dihedral $\mathbf{T}_{D}$ and volume $\mathbf{T}_{V}$ scattering. The first component in Eq. (3.1) represents direct backscatter from a smooth surface $(k s<0.3)$, which can be expressed by the SPM or Bragg scattering model (Yamaguchi et al. 2006):

$$
\begin{aligned}
& \mathbf{T}_{S}=f_{S}\left[\begin{array}{ccc}
1 & \beta_{S}^{*} & 0 \\
\beta_{S} & \left|\beta_{S}\right|^{2} & 0 \\
0 & 0 & 0
\end{array}\right] \text { with } \\
& f_{S}=\frac{m_{S}^{2}}{2}\left|R_{h}+R_{v}\right|^{2} \text { and } \beta_{S}=\frac{R_{h}-R_{v}}{R_{h}+R_{v}}
\end{aligned}
$$

where the coefficients $R_{h}$ and $R_{v}$ are the horizontal and vertical Bragg scattering coefficients and $m_{S}$ represents the soil roughness influence on the intensity component $f_{S}$. Both Bragg scattering coefficients depend only on the dielectric constant of the soil $\varepsilon_{S}$ and the local incidence angle $\theta_{l}$.

The dihedral scattering component can be modelled as a double Fresnel reflection on smooth dielectric media leading to the following rank-1 coherency matrix (Yamaguchi et al. 2006) and including the dihedral scattering intensity $f_{D}$ and the dihedral scattering mechanism ratio $\alpha_{D}$ :

$$
\begin{aligned}
& \mathbf{T}_{D}=f_{D}\left[\begin{array}{ccc}
\left|\alpha_{D}\right|^{2} & \alpha_{D} & 0 \\
\alpha_{D}^{*} & 1 & 0 \\
0 & 0 & 0
\end{array}\right], \text { with } \\
& f_{D}=\frac{m_{D}^{2}}{2}\left|R_{s h} R_{t h}+R_{s v} R_{t v} e^{i \varphi_{D}}\right|^{2} \text { and } \\
& \alpha_{D}=\frac{R_{s h} R_{t h}-R_{s v} R_{t v} e^{i \varphi_{D}}}{R_{s h} R_{t h}+R_{s v} R_{t v} e^{i \varphi_{D}}}
\end{aligned}
$$

where the horizontal and vertical Fresnel coefficients of the soil plane $\left(R_{s h}, R_{s v}\right)$ and of the trunk plane $\left(R_{t h}, R_{t h}\right)$ depend on the soil and trunk dielectric constant $\left(\varepsilon_{S}\right.$ and $\left.\varepsilon_{t}\right)$ and the respective incidence angle $\theta_{s}=\theta_{l}$ and $\theta_{t}=\pi / 2-\theta_{l}$. The phase $\varphi_{D}$ incorporates differential propagation in the case of an orientated vegetation layer, while $m_{D}$ represents the scattering loss on the intensity component $f_{D}$. 
The most challenging component with respect to EM modelling is the vegetation volume. A widely used approach deals with the vegetation volume as a cloud of equally shaped particles with a certain orientation distribution. In one of the simplest ways, the volume component is modelled as randomly oriented cloud of dipoles. The initial vertical dipole can be expressed with the following scattering matrix $\mathbf{S}_{v d i}$ :

$$
\mathbf{S}_{v d i}=\left[\begin{array}{ll}
0 & 0 \\
0 & 1
\end{array}\right]
$$

After expansion to the coherency matrix, the matrix is rotated around the LoS by an angle of $2 \psi$ to account for the orientation of the scattering particles:

$$
\mathbf{T}_{r o t}=\mathbf{R}_{2} \mathbf{T}_{v d i} \psi \mathbf{R}_{2 \psi}^{T}=\left[\begin{array}{ccc}
1 & 0 & 0 \\
0 & \cos 2 \psi & \sin 2 \psi \\
0 & -\sin 2 \psi & \cos 2 \psi
\end{array}\right]\left[\begin{array}{ccc}
1 / 2 & -1 / 2 & 0 \\
-1 / 2 & 1 / 2 & 0 \\
0 & 0 & 0
\end{array}\right]\left[\begin{array}{ccc}
1 & 0 & 0 \\
0 & \cos 2 \psi & -\sin 2 \psi \\
0 & \sin 2 \psi & \cos 2 \psi
\end{array}\right]
$$

In order to respect all the different orientations occurring within vegetation, the rotated coherency matrix $\mathbf{T}_{r o t}$ is integrated together with the specific probability density function $p_{\psi}(\psi)$ of orientations assuming a uniform angular distribution $p_{\psi}(\psi)=1 /(2 \pi)$ and a distribution width $\Delta \psi=\psi_{2}-\psi_{1}=2 \pi$ in the case of a randomly oriented vegetation volume (Yamaguchi et al. 2006):

$$
\begin{aligned}
\mathbf{T}_{V} & =f_{V} \int_{\psi_{1}}^{\psi_{2}} p_{\psi}(\psi) \mathbf{T}_{r o t} d \psi \\
& =f_{V}\left[\begin{array}{ccc}
1 / 2 & 0 & 0 \\
0 & 1 / 4 & 0 \\
0 & 0 & 1 / 4
\end{array}\right] .
\end{aligned}
$$

In a first step, the modelled vegetation volume component $\mathbf{T}_{V}$ with its volume intensity $f_{V}$ is subtracted from the measured polarimetric SAR data $\mathbf{T}$ to separate the volume $\mathbf{T}_{V}$ and the ground $\mathbf{T}_{G}$ components $\left(\mathbf{T}_{S}+\mathbf{T}_{D}\right)$ :

$$
\langle\mathbf{T}\rangle-f_{V} \mathbf{T}_{V}=\mathbf{T}_{G}=\mathbf{T}_{S}+\mathbf{T}_{D} .
$$

However, if the volume modelling is imperfect, Eq. (3.6) leads to biased or even non-physical results for the ground component $\mathbf{T}_{G}$. To avoid this effect, a mathematical method using the positive semi-definiteness of the Hermitian ground component was proposed by $\mathrm{Van} \mathrm{Zyl}$ et al. to correct the volume intensity $f_{V c o r r}$ (Van Zyl et al. 2008). With this corrected volume intensity, the linear system of Eq. (3.2) is solved for the single ground components $\left(\alpha_{D}, \beta_{S}, f_{S}, f_{D}\right)$ of the three scattering contributions obeying the dominance criterion of Freeman et al. for the ground components (Freeman and Durden 1998).

\subsubsection{Experimental Results}

Test sites and corresponding radar and validation data sets selected for crop classification are summarised in Table 3.4. The Indian Head test site is further described in the Appendix.

The presented polarimetric methodology was employed on the fine quad-polarimetric RADARSAT-2 data sets of Flevoland and Indian Head in order to decompose the SAR data into their respective scattering components (surface, dihedral, volume) and afterwards to invert the volumetric soil moisture from the ground components (surface, dihedral).

Figure 3.7 displays exemplarily the RGB composite of the normalised decomposed scattering power components from the three-component model-based decomposition introduced in Sect. 3.3.2 for the two test sites and two different acquisition dates (Flevoland, in the beginning of the observation cycle, 11th of June 2009, towards the end of the observation cycle, 29th of August 2009; Indian Head, 4th of August and 4th of September 2009). For the Flevoland scenes, the water bodies in blue colour and the urban areas predominantly in red colour are clearly visible in both scenes due to their temporal scattering stability. However, the agricultural areas are mostly indicated by the rectangular "patch work" structures across the centre of the scenes, which change significantly between the two acquisitions. For the Indian Head scenes, the agricultural fields show two different patterns for each date (August, September) due to the phenological plant development along the growing cycle. While the strongly vegetated fields exhibit a strong volume scattering, the low vegetated (catch crops) or bare fields (after harvest) indicate predominantly surface scattering. 
Table 3.4 Test sites and corresponding radar and validation data selected for the generation of showcases on soil moisture estimation under vegetation

\begin{tabular}{|c|c|c|}
\hline Application/product & Test site - radar data & Reference data \\
\hline \multirow{6}{*}{$\begin{array}{l}\text { Soil moisture estimation } \\
\text { under vegetation }\end{array}$} & Flevoland, the Netherlands & \multirow{3}{*}{$\begin{array}{l}\text { Two continuously recording soil moisture stations (volumetric soil moisture } \\
\text { content, hourly sampling from } 26 / 05-06 / 10 / 2009 \text { ) }\end{array}$} \\
\hline & $\begin{array}{l}\text { RADARSAT-2, C-band - fine quad- } \\
\text { polarimetric SLC data sets }\end{array}$ & \\
\hline & $\begin{array}{l}\text { Time series from April } 2009 \text { to } \\
\text { August } 2009\end{array}$ & \\
\hline & Indian Head, Canada & \multirow{3}{*}{$\begin{array}{l}\text { One continuously recording soil moisture station (volumetric soil moisture } \\
\text { content, hourly sampling from 17/07-13/09/2009) }\end{array}$} \\
\hline & $\begin{array}{l}\text { RADARSAT-2, C-band - fine quad- } \\
\text { polarimetric SLC data sets }\end{array}$ & \\
\hline & $\begin{array}{l}\text { Time series from July } 2009 \text { to } \\
\text { September } 2009\end{array}$ & \\
\hline
\end{tabular}

In June, after the start of the crop cycle (Fig. 3.7 left), the fields appear in all three colours, indicating a mixture of surface (bare soil), dihedral (stalk/leave-ground) and volume (complex vegetation) scattering due to the different phenologies of the different crop types, but also the different sowing dates on different fields.

In August, within the final stage of the crop cycle (Fig. 3.7 right), a part of the agricultural fields (mostly the winter crops) are already harvested and appear in blue colour indicating predominantly surface scattering, while the other part of the agricultural fields (summer crops, like corn) still exhibit green colours, indicating complex volume scattering before harvesting.

Figure 3.8 exhibits the trends of the three normalised scattering power components (red, dihedral; green, volume; blue, surface) for Flevoland and Indian Head test sites. The general trend is similar for both test sites at Flevoland, where volume is dominant most of all times with values around 0.6-0.7 peaking in July/August during the maturity stage of the vegetation. The ground components always stay below 0.4-0.5 except for the April dates in Flevoland.

Moreover, dihedral scattering is mostly present within the June dates in Flevoland, when the vegetation is active and fresh for a distinct double bounce and almost vanishes afterwards, while surface scattering dominates over dihedral scattering for Flevoland in July and August 2009. For the Indian Head test site, the time series in Fig. 3.8 reveals a clear dominance of volume scattering (level $=0.8$ along all August) due to a strong agricultural vegetation cover of canola crops (vegetation height $>1.0 \mathrm{~m}$ ) on the test field, where some minor ground scattering occurs as single-bounce surface and/or as double-bounce dihedral scattering (level $<0.2$ ). Hence, the dry moisture regime (mean soil moisture level lower than $8 \mathrm{vol} . \%$ ) together with the thick vegetation layer of canola will be a big challenge for soil moisture inversion under vegetation at $\mathrm{C}$-band.

In conclusion, the decomposition led to a physically meaningful separation of the scattering contributions within the C-band RADARSAT-2 scenes, which reflects the agricultural scattering scenario reasonably well. After the model-based polarimetric decomposition into the single scattering components, the surface and dihedral components were inverted for soil moisture retrieval under vegetation cover as described in Sect. 3.3.2. Only for Flevoland very low inverted moisture ranges (soil moisture $<5.6 \mathrm{vol} . \%$ ) compared to the in situ measured moistures ( 30-40vol.\%) are not representative and are filtered out in the end of the inversion process.

Figure 3.9 presents exemplarily the combination of inverted soil moisture values from both ground components (surface, dihedral) for the Flevoland (11th of June 2009 and 29th of August 2009) and Indian Head (4th of August and 4th of September 2009) test sites. The inversion rates for the whole Flevoland scene are $33.12 \%$ for the June date and $32.70 \%$ for the August date, while the inversion rates for both test sites and all scenes are displayed in Fig. 3.10. For Flevoland the inversion rates vary between $22 \%$ and $33 \%$ along time, whereby the minimum is reached in July, when the agricultural vegetation is in its maturity stage. For Indian Head the inversion rate varies between $11 \%$ and $25 \%$ growing slightly higher in the end of the vegetation growth period. Hence, in all cases, the inversion exhibits gaps of non-invertible points (white areas in Fig. 3.9). The inversion mostly failed within the agricultural regions due to the variety of scattering scenarios (typical field sizes at Flevoland: 10-15 ha), which are difficult to describe in detail with one type of model-based polarimetric decompositions assuming just a randomly oriented vegetation volume.

However, distinct field heterogeneities are visible within the soil moisture results, which fit the expectations of a highly varying soil moisture content on the agricultural fields. For Indian Head, a higher inversion rate can be stated for the scene acquired in September due to a diminished vegetation cover caused by harvesting activities and senescence.

In general, the inversion result at Flevoland for the 11th of June 2009 reveals a higher soil moisture level on the dihedral dominant fields than on the fields with surface scattering. In addition, the dominance of surface scattering for the 29th of 
a
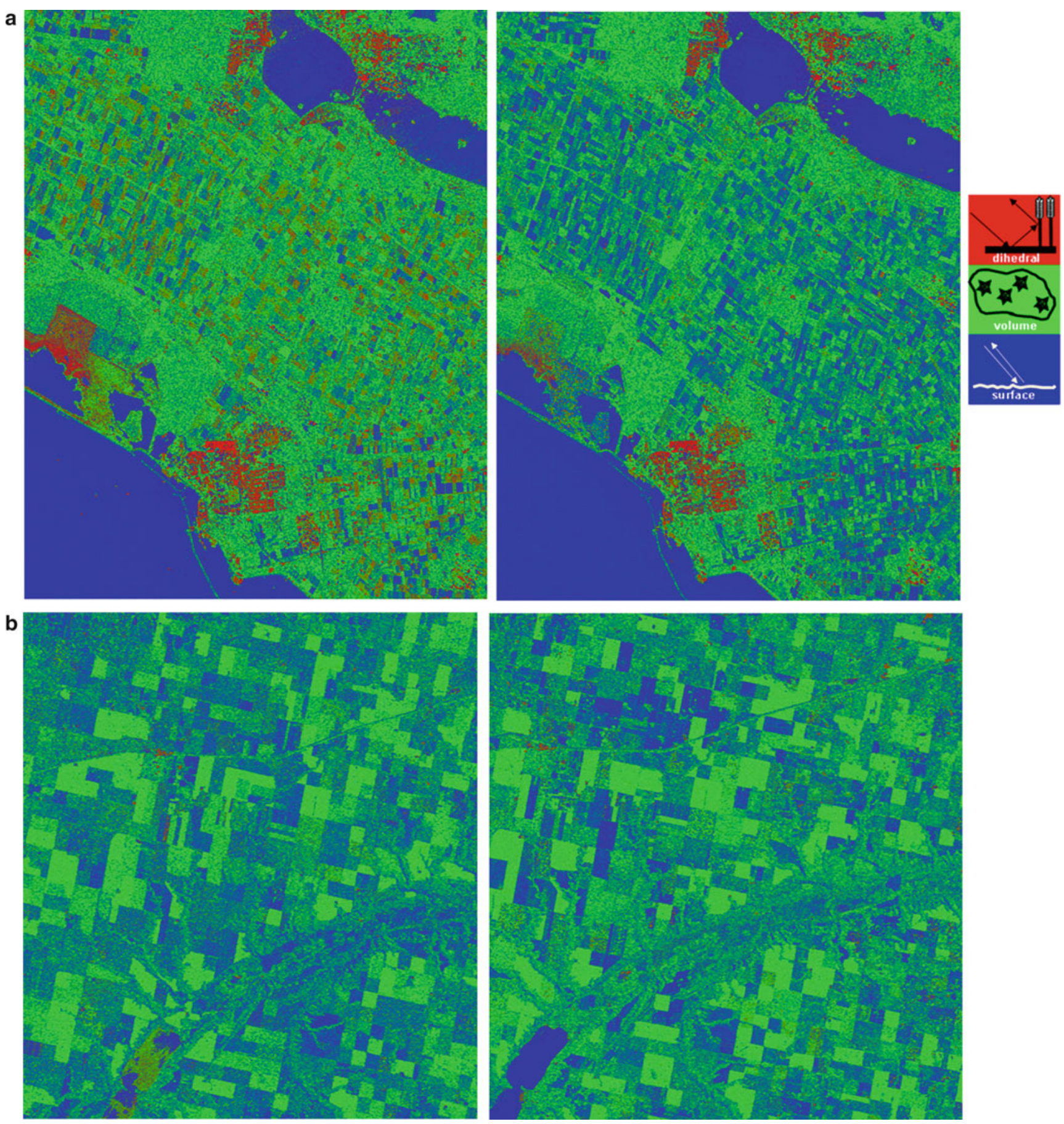

Fig. 3.7 RGB composite of normalised decomposed scattering power components from model-based polarimetric decomposition for the beginning of the observation cycle at 11th of June 2009 (Top left) and the end of the observation cycle at 29th of August 2009 (Top right) at the Flevoland test site and for 4th of August in the main growth period

(Bottom left) and 4th of September 2009 in the end of the main growth period (Bottom right) at the Indian Head test site (red, even bounce/ dihedral scatterers; green, volume scatterers; blue, odd bounce/surface scatterers)

August 2009 seems to result in a lower moisture level on most of the fields. In contrast to Flevoland, the Indian Head test site exhibits generally a lower soil moisture level, but also states relatively a higher moisture regime for fields with dihedral compared to surface scattering.

Figure 3.11 presents the validation of the inverted soil moisture from the ground scattering components (surface, dihedral) with the in situ measurements of the 2 test sites and all continuously recording moisture stations for 11 different dates from 11th of June until the 29th of August 2009 at 

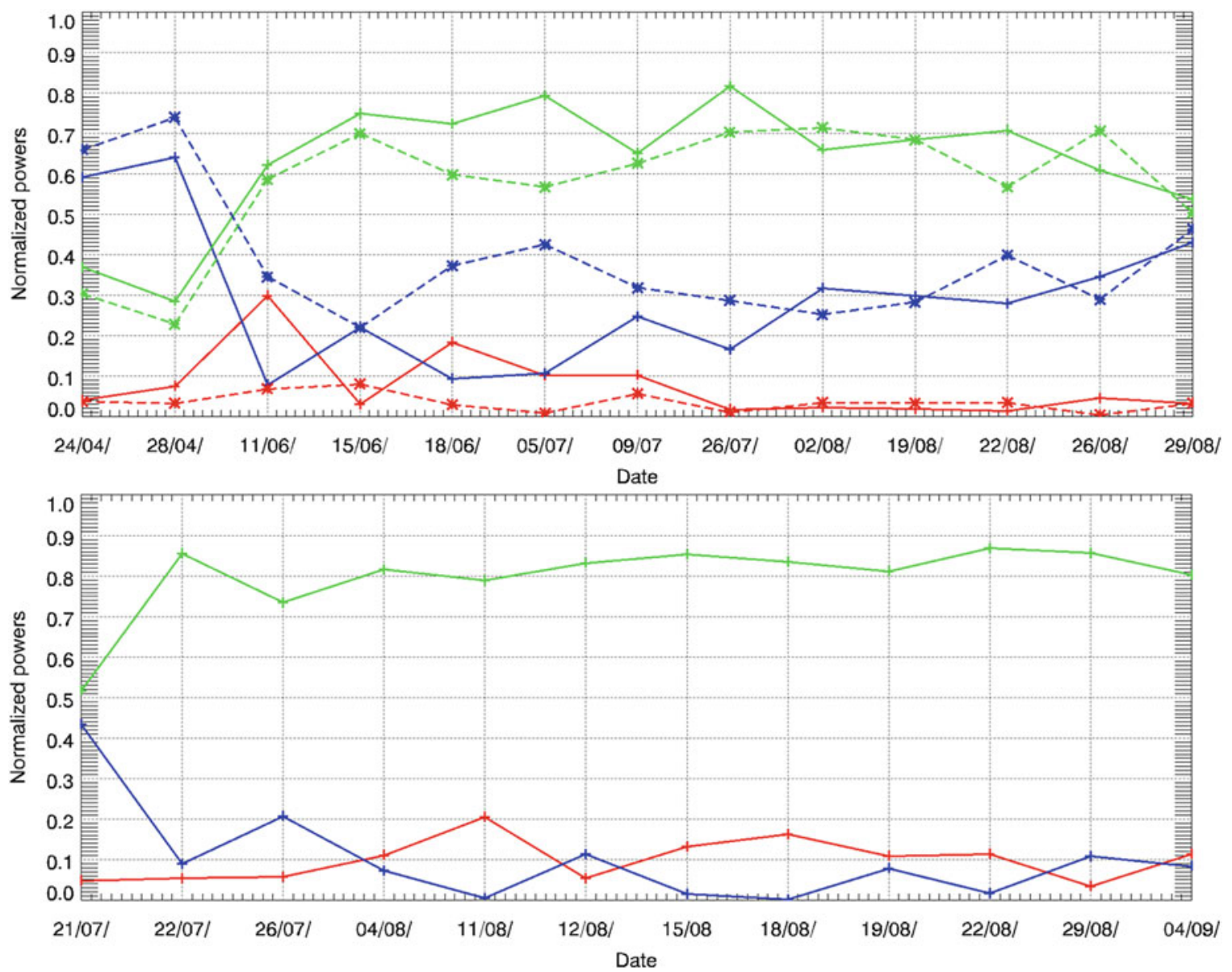

Fig. 3.8 Normalised decomposed scattering power components (red, dihedral; green, volume; blue, surface) from the model-based polarimetric decomposition for the Flevoland test sites (site 1, Plus sign; site 2 , Star sign from the beginning of the observation cycle at 11th of June

Flevoland and for 9 dates from 4th of August until 4th of September 2009 at Indian Head. A clear underestimation has to be stated at Flevoland for all dates of the observation cycle. In contrary, a distinct overestimation is reported by the comparison with in situ measurements at Indian Head. The estimated soil moisture values for the two measurement locations at Flevoland are underestimating the measured soil moisture values by a RMSE of 13.32 vol. $\%\left(r^{2}=0.12\right)$, whereas the comparison with the field measurements for Indian Head overestimates with a RMSE of 22.84vol.\% $\left(r^{2}=0.02\right)$ including all analysed dates.

In the past, the soil moisture retrieval method was developed and implemented for L-band data (see Jagdhuber et al. 2012b, 2013a, b), and with the transfer to the C-band scattering scenario, the algorithm needs further adaptions especially concerning the ground scattering models. First analyses on the surface component inversion blame the low moisture values at Flevoland on the low $\mathrm{T}_{12}$ correlation term, which
2009 until the end of the observation cycle at 29th of August 2009) and for the Indian Head test site (site 3, Plus sign) (R, even bounce/dihedral scatterers; G, volume scatterers; B, odd bounce/surface scatterers)

leads to small $\beta_{S}$ values and the underestimation of the soil moisture level.

In addition, the measured soil moisture of the two in situ stations at Flevoland ranges between $24 \mathrm{vol} \%$ and $42 \mathrm{vol} \%$. This represents a significantly high soil moisture level, which is an additional challenge for the inversion algorithm, because the sensitivity of the inversion models decreases with increasing soil moisture level (Jagdhuber 2012).

For Indian Head, the distinct overestimation of the soil moisture might be traced back to a very challenging scattering scenario of a large canola vegetation cover $(>100 \mathrm{~cm})$ and a very dry soil underneath, which might be hardly detectable at C-band (wavelength: $5 \mathrm{~cm}$ ).

Moreover, it has to be stated that the validation could only be conducted over time (continuously recording moisture stations) and not over space (no soil moisture network or spatial sampling), since only three measurement locations were available for Flevoland and Indian Head in 2009. 

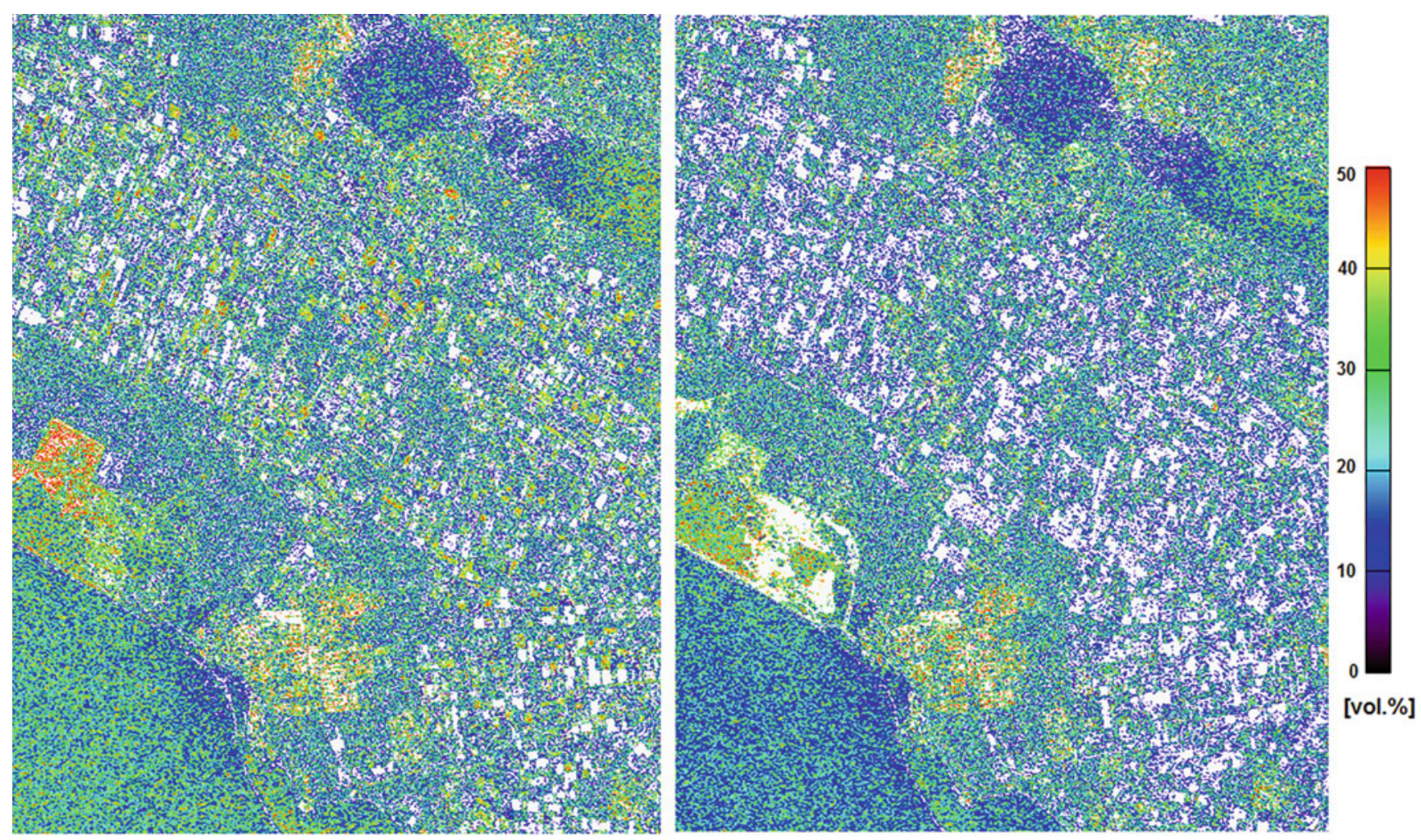

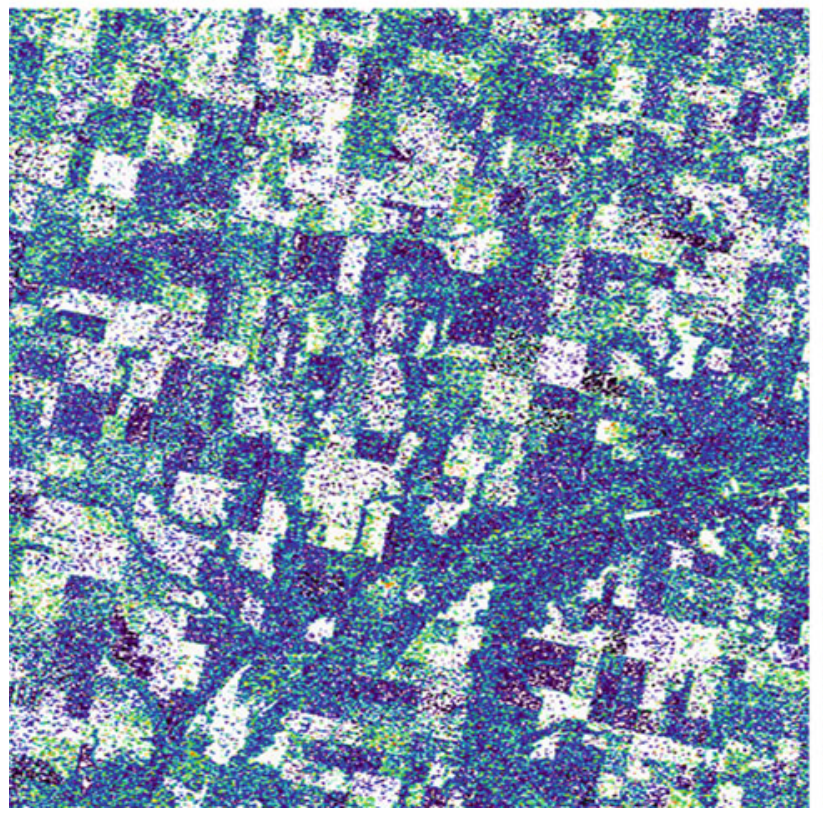

Fig. 3.9 Inverted soil moisture [vol.\%] from both ground scattering components (surface, dihedral) for the Flevoland test site (at the beginning of the observation cycle at 11th of June 2009 (Top left) and the end of the observation cycle at 29th of August 2009 (Top right)) and for the

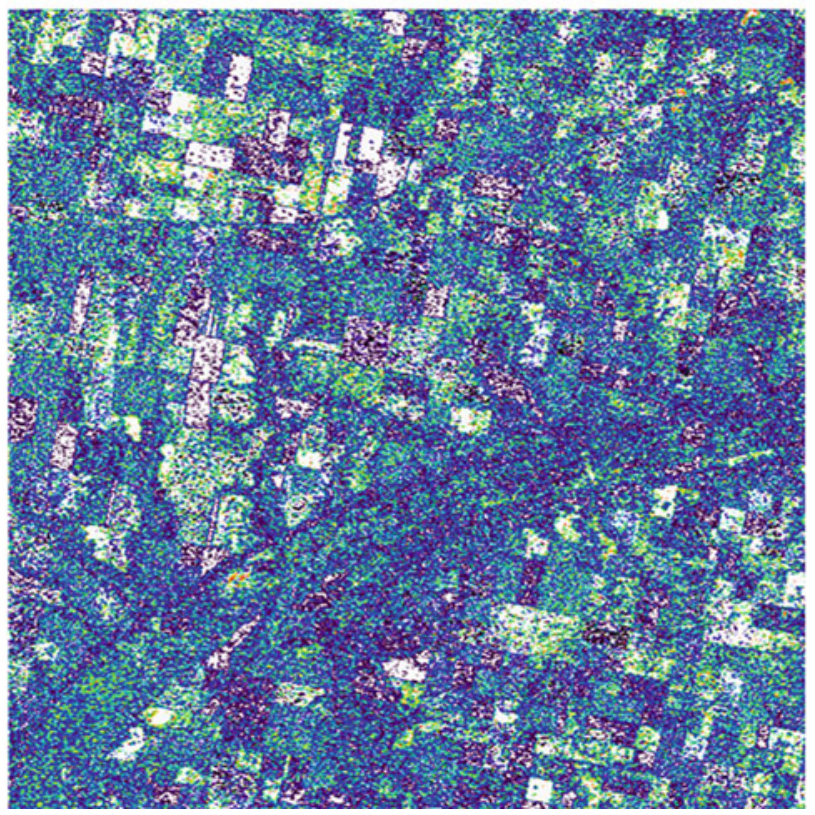

Indian Head test site (on the 4th of August (Bottom left) and the 4th of September (Bottom right) 2009); non-invertible areas are masked white; image smooth: $4 \times 4$ 


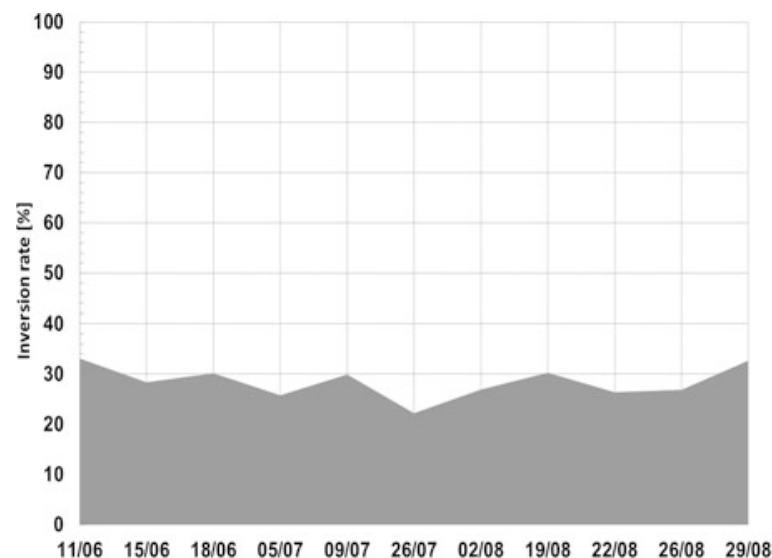

(a)

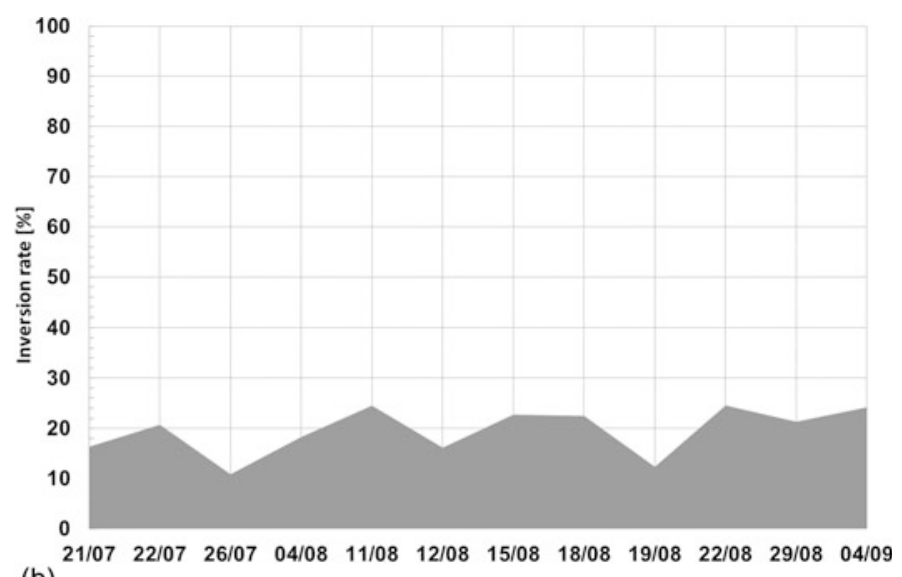

(b)

Fig. 3.10 Inversion rate [\%] for soil moisture retrieval of both ground scattering components (surface, dihedral) at Flevoland (left) from the beginning of the observation cycle at 11th of June 2009 until the end of

the observation cycle at 29th of August 2009 and at Indian Head (right) from 21st of July until 4th of September 2009

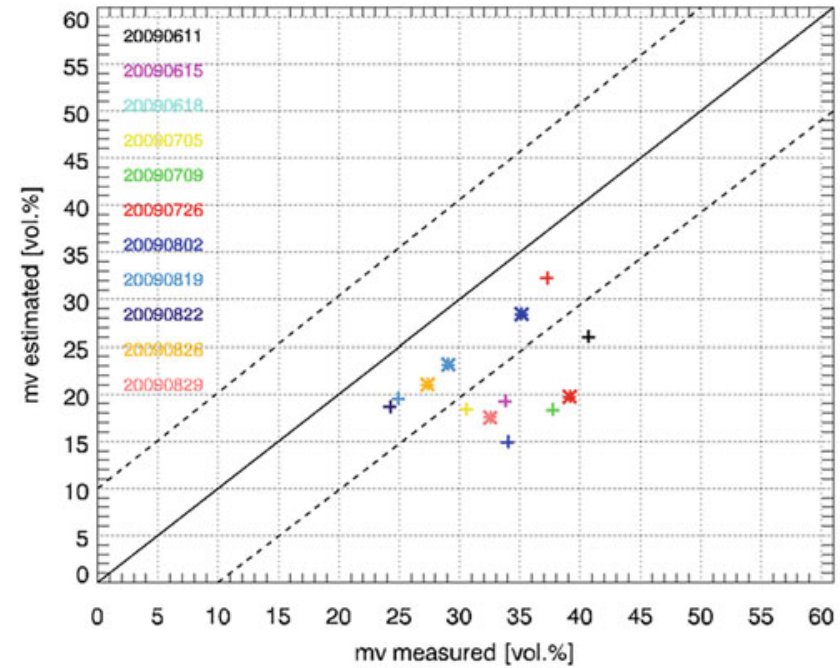

Fig. 3.11 Validation of inverted soil moisture [vol.\%] from ground scattering components (surface, dihedral) with in situ measurements of soil moisture at the two continuously recording moisture stations (site 1, Plus sign; site 2, Star sign) at Flevoland (left) for 11 different dates

\subsubsection{Comparison with Single-/Dual-Pol Data}

The developed algorithm is directly and solely based on the fully polarimetric observation space and therefore directly affected by the reduction to single- or dual-polarimetric data. This means that only in the case of fully polarimetric data, the vegetation- and/or soil roughness-induced depolarisation can be assessed, quantified and removed in the best way. A decomposition, separating the vegetation

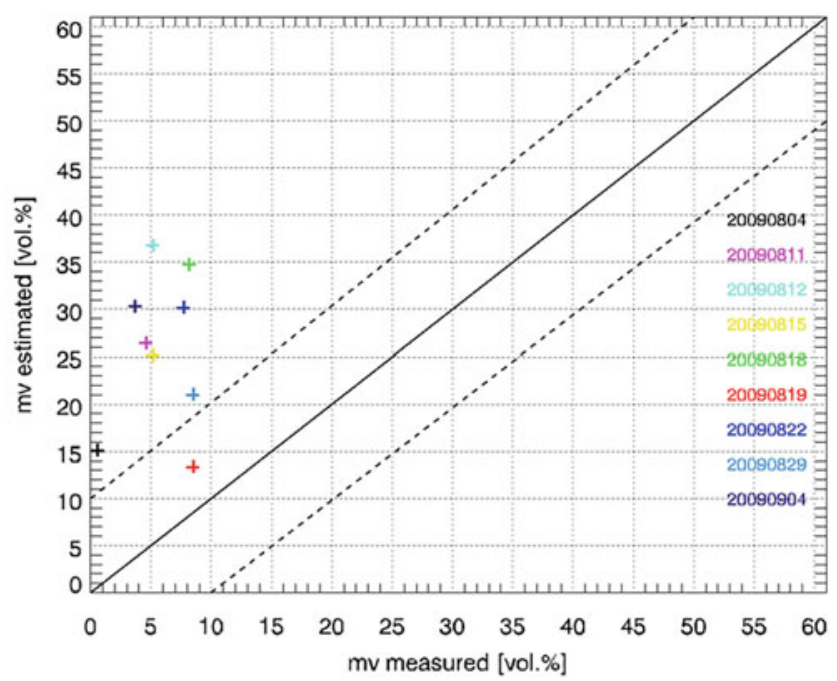

from 11th of June until the 29th of August 2009 (colours) and at Indian head (right) for 12 dates from 4th of August until 4th of September 2009; validation box: $7 \times 7$ pixels

volume and the soil ground scattering components, is not/only partly feasible with single-/dual-polarimetric approaches.

Hence, there is a trade-off between decomposing fully polarimetric data and then solving a lower parameterised inversion problem or using single-/dual-polarimetric data and modelling the whole backscatter signal by solving an ambiguous, highly parameterised inversion problem. 

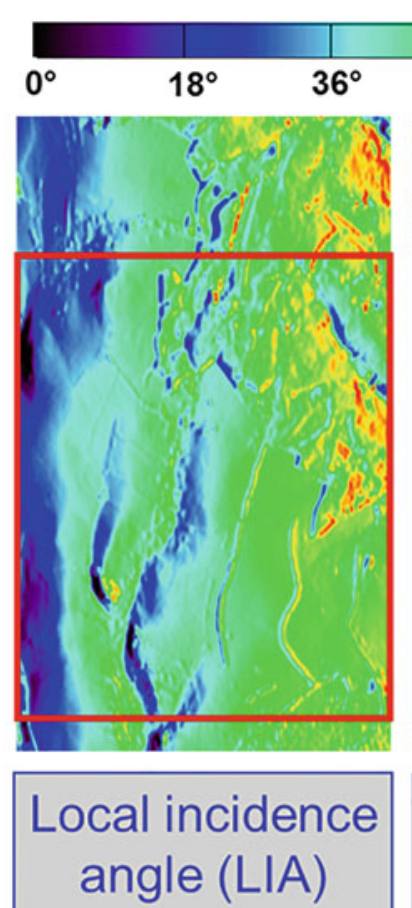

master

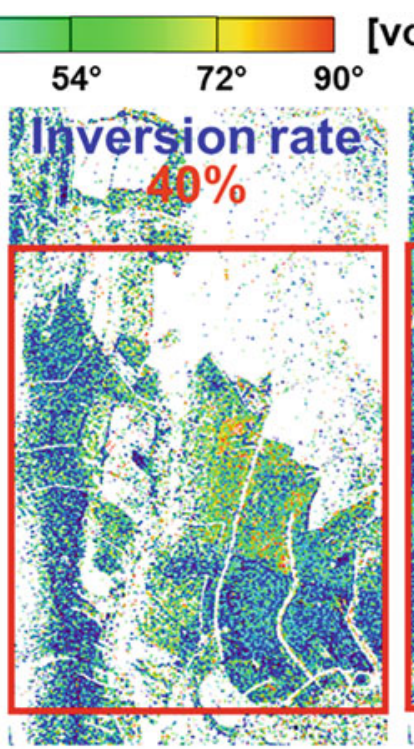

Single angular

1 acquisition

master $(\mathrm{m})$

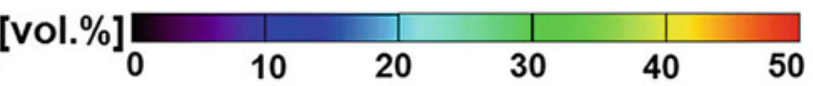
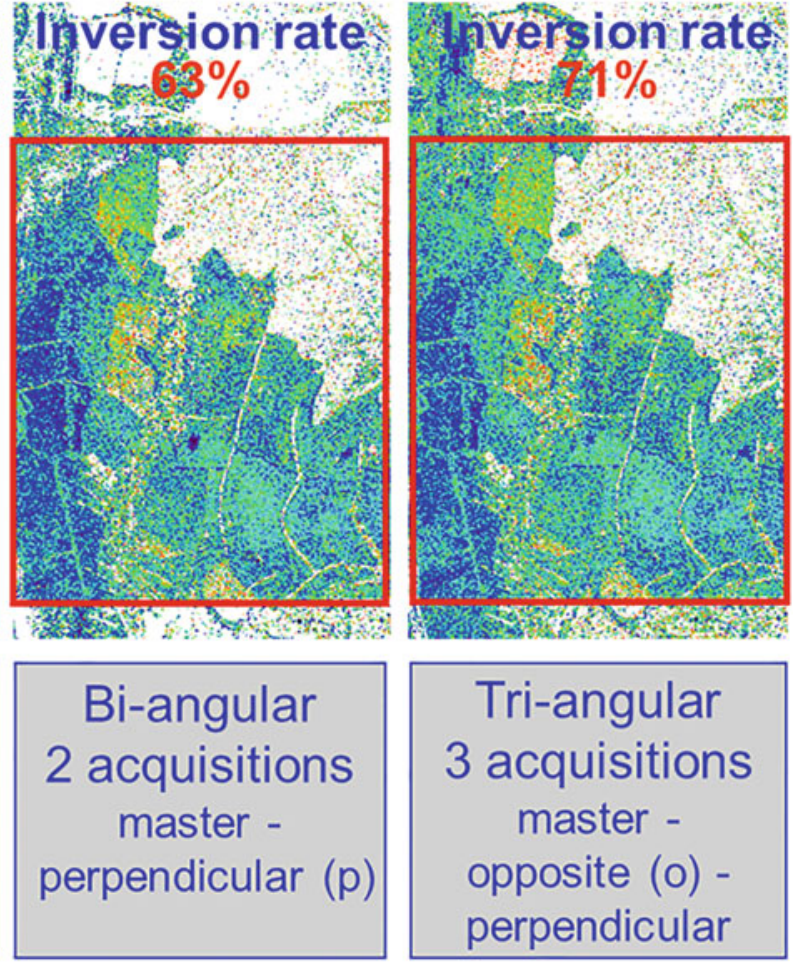

Tri-angular 3 acquisitions master opposite (o) perpendicular

\section{Tri-angular: Master-opposite-perpendicular}
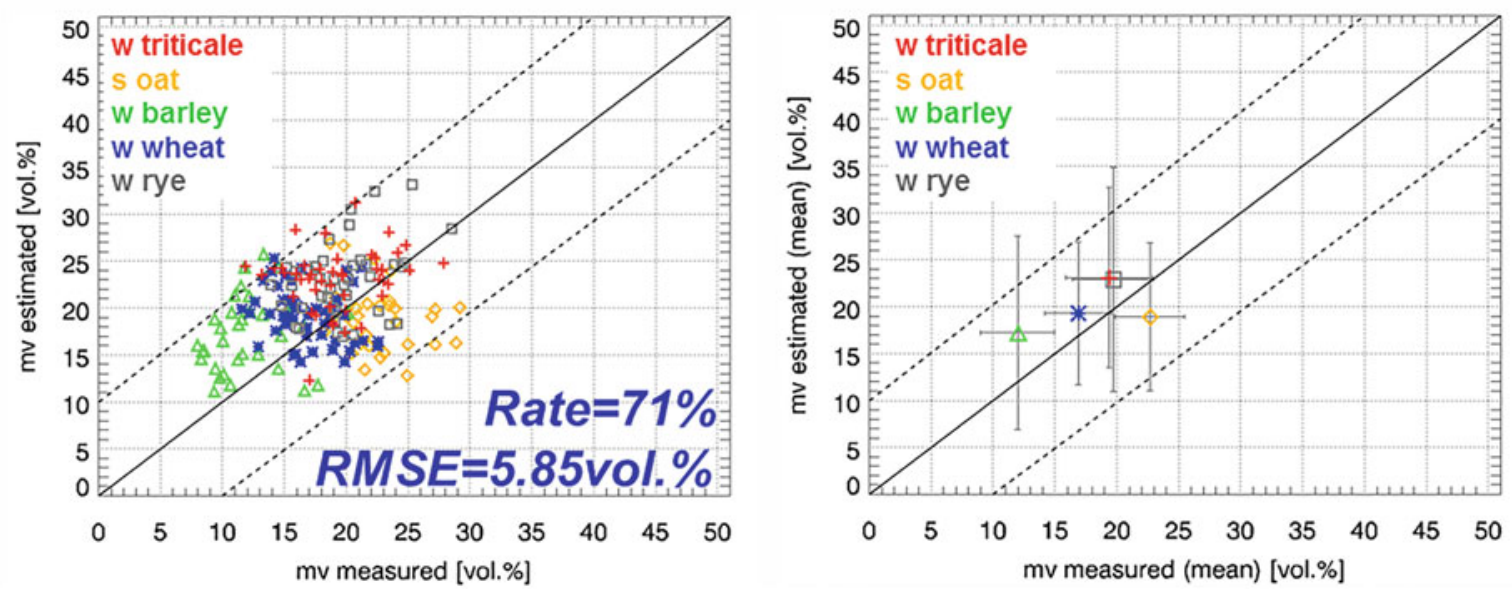

Fig. 3.12 Multi-angular polarimetric decomposition for soil moisture retrieval under vegetation cover at L-band applied to E-SAR data of the OPAQUE 2008 campaign: (Top) Local incidence angle and soil moisture inversion results for a single-, bi- and tri-angular model-based decomposition and inversion are depicted. The inversion rate constantly

increases, when moving to a multi-angular decomposition and inversion. (Bottom) Validation of the SAR-based moisture estimates with in situ measurements from different crop types for each single measurement location (left) and for the mean of field values (right) (Jagdhuber et al. 2013a) 

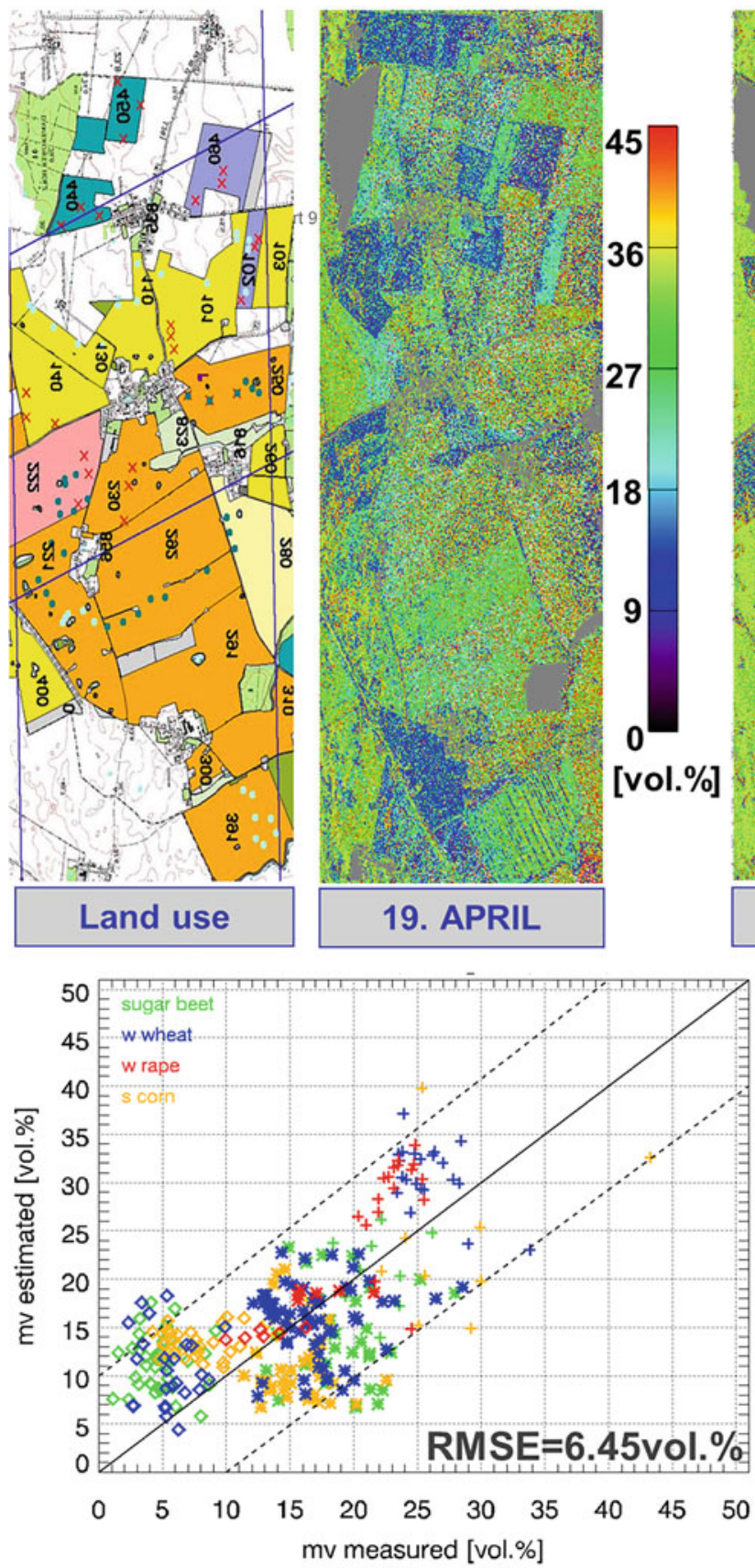

Fig. 3.13 Hybrid polarimetric decomposition for soil moisture retrieval under vegetation cover at L-band applied to E-SAR data of the AgriSAR 2006 campaign: (Top) Land use and soil moisture inversion results for a time series covering the entire vegetation cycle (April-July 2006) are depicted. Urban and forested areas are masked grey. (Bottom)

\subsubsection{Discussion on the Role of Polarimetry, on the Maturity of the Application and Conclusion}

Polarimetry and the acquisition of fully polarimetric SAR data are essential for the retrieval of soil moisture under vegetation. Only the polarimetric observation space with its
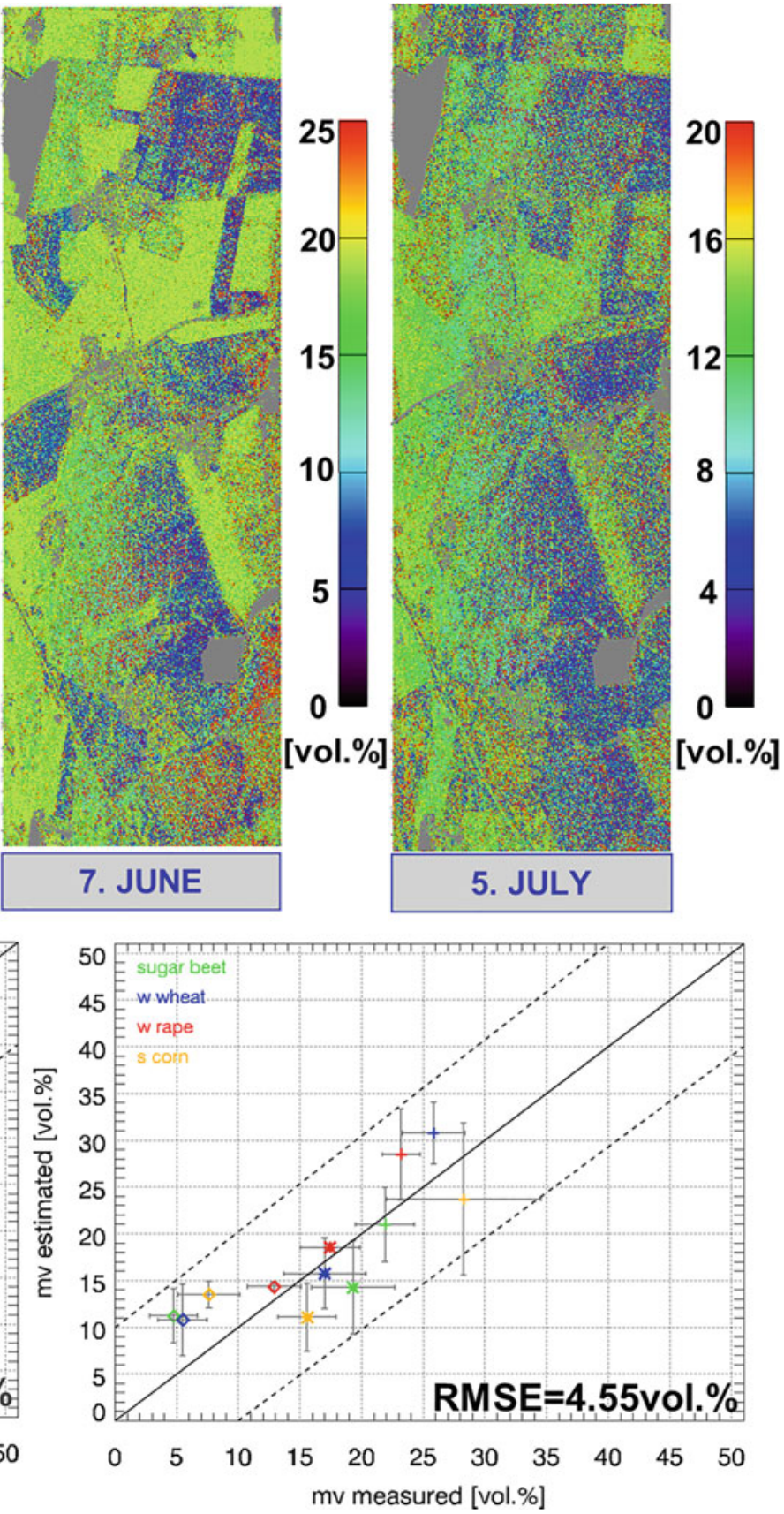

Validation of the SAR-based moisture estimates with in situ measurements from different crop types for each single measurement locations (left) and for the mean of field values (right). Symbology: Diamond, 5th of July; Stars, 7th of June; Plus, 19th of April) (Jagdhuber et al. 2013b, 2014)

capability of polarimetric decompositions provides and reveals the different properties (dielectric content, object geometry) of the scatterers on ground in the most complete way compared to backscatter intensity analyses or interferometric techniques. Therefore SAR polarimetry plays an essential role in this application. 
In the last years, the application of soil moisture retrieval under vegetation advanced to a distinct level of maturity by using longer wavelength SAR (L-band) (Jagdhuber et al. 2012b, 2013a, b). Compared to C-band, L-band provides a deeper penetration into the vegetation canopy together with a convenient signal-to-noise ratio (SNR) for reliable polarimetric signal analyses (Jagdhuber 2012). Figures 3.12 and 3.13 show exemplarily two novel and innovative types of polarimetric decompositions for soil moisture inversion under vegetation at longer wavelength (L-band): a multi-angular, model-based decomposition and a hybrid (combined model-/eigen-based) decomposition. The multi-angular decomposition in Fig. 3.12 leads to the increase in inversion rate from $40 \%$ to $71 \%$, when moving from a single-angular to a tri-angular model-based decomposition, which also reflects in the quality of the inversion with an RMSE of 5.85vol.\% in contrast to the C-band results (in Fig. 3.11) (Jagdhuber et al. 2013a). In comparison to all presented inversion approaches, Fig. 3.12 reveals the best inversion and validation results of the novel hybrid decomposition technique using a modelbased decomposition for the volume removal and an eigenbased decomposition to retrieve the single ground scattering components for soil moisture inversion under vegetation cover for an entire vegetation growth period sensed during the AgriSAR 2006 campaign (Jagdhuber et al. 2013b). The inversion rate is always higher than $95 \%$ including the entire vegetation cycle. Moreover, the RMSE for all acquisition dates (April-July 2006) lies in the favourable range of $4,6 \mathrm{vol} . \%$ (mean of field values) to $6.5 \mathrm{vol} . \%$ (single scattering locations) for ecosystem applications.

Furthermore, first attempts were also made for retrieving soil moisture under boreal forest using P-band in order to enlarge the moisture retrieval to forested environments (Jagdhuber et al. 2012a). In addition, also X-band fully polarimetric data of TerraSAR-X are currently investigated for soil moisture retrieval on bare and sparsely vegetated soils for shorter wavelength SAR (Jagdhuber et al. 2013c, d). In this way, the fully polarimetric soil moisture estimation using decomposition techniques is approached covering the frequency range from $\mathrm{X}$ - to $\mathrm{P}$-band considering the wavelength-dependent sensitivity for vegetation cover and the soil below.

\subsection{Crop Phenology Estimation Using SAR Polarimetry}

\subsubsection{Introduction, Motivation and Literature Review}

All agricultural crops present a continuous development, from sowing or transplanting to harvest, in which they grow and evolve in accordance with their biophysical characteristics and the farming practices applied to them. Phenology denotes such a succession of stages during the cultivation cycle and is commonly expressed using numerical scales (Meier 2001).

Tracking phenology of agricultural fields by remote sensing is useful for farmers with extensive fields because it provides key information for planning and triggering cultivation practices, so the main application of this EO product is precision farming. These cultivation practices (e.g. irrigation, fertilisation, effective germination counting, harvest, etc.) require timely inputs about the status of the plants and, specifically, about their condition or situation along the expected cultivation cycle.

Besides precision farming, timely information of phenology can contribute to agencies and institutions involved in market predictions, insurance policies, subsidies claims, etc. since such information complements their own data sources and provides a temporal schedule for the crop production and yield calendar.

Most of the applications require phenology information at parcel (field) level, but in some cases it may be necessary to provide values at pixel (sub-parcel) level, especially when dealing with detection of heterogeneities produced by cultivation problems (e.g. water salinity used of irrigation) and plant diseases (e.g. pests and plagues). Unfortunately phenology monitoring by satellite remote sensing has not attracted much attention in the past due to both the lack of time series of images and the cost of the ground campaigns. Nevertheless, this situation has changed in recent times, thanks to the launch of satellites with shorter revisit times (e.g. 11 days for TerraSAR-X) and reconfigurable acquisitions (different beams can be operated for more frequent observation of a particular area). Importantly for us these are also available in multiple polarisation channels, which prompted this research into phenology retrieval using polarimetric imaging radar (PolSAR).

Algorithms for phenology monitoring have to be devised individually for each crop type, based on the expected response of each crop to the sensor at its different stages, which can be extracted from the data themselves (training sets) or from previous experiments, models and simulations. In addition, approaches based on the availability of time series of images can improve importantly the performance of these algorithms, since phenology is intrinsically related to time.

The starting point of this application is the knowledge of which crop type or plant species is cultivated at the monitored fields, which is provided by the users or can be obtained from a crop type map. Then, the general objective of this product consists in estimating the current phenological stage of the plants in the parcel by exploiting a single PolSAR acquisition.

The topic of vegetation phenology monitoring by means of remote sensing has been mostly addressed in the past by 
analysing temporal variations of vegetation indices acquired by optical sensors. These vegetation indices are formed by combining different spectral bands which exhibit different sensitivities to biochemical plant constituents and canopy biophysical parameters. These indices are then used as a proxy for monitoring the plant growth stage by means of empirical relationships with structural parameters. Among all of them, the most important one is LAI (leaf area index) which plays a key role in the design of crop models for crop condition assessment and final yield prediction (Bach and Mauser 2003).

In addition to different airborne sensors operating worldwide (CASI, ROSIS, DAIS-7915), there are several operative satellite missions which have been providing multispectral data for large-scale Earth dynamics studies. Some of these instruments are the MODerate-resolution Imaging Spectroradiometer (MODIS) by NASA, the Advanced Very High Resolution Radiometer (AVHRR) by NOAA and the VEGETATION sensor on board SPOT satellite by CNES. In general, the main purpose of all these projects has been primarily focused on crop yield prediction and crop mapping/classification, being these tasks directly related to the estimation of phenology (Nellis et al. 2009).

Early studies by Badhwar and Henderson in 1981 (Badhwar and Henderson 1981) made use of Landsat Multispectral Scanners (MSS) in order to design a crop model able to determine development stage of corn and soybean. Further improvement was proposed by Tilton and Hollinger in 1982 (Tilton and Hollinger 1982) when they proposed an algorithm to predict the development stages early in the growing season by means of a principal component analysis of Landsat images. In 1990, Lloyd exploited the approximately linear relationship between solar energy absorption of plants and NDVI measurements acquired by NOAA-AVHRR in order to describe phenological events (Lloyd 1990). Likewise, in 1994 Reed et al. (1994) analysed time series of NOAA-AVHRR acquisitions as well, and they found a high correlation between NDVI temporal trends and phenological variations in case of spring wheat at a continental scale.

The work by Tucker in 1979 (Tucker 1979) is considered as one of the first contributions that proposed the systematic use of NDVI for characterising vegetation. Since then, NDVI has been the most widely used spectral index for vegetation monitoring. It combines the reflectance values of plants in red (low reflectance) and near-infrared (high reflectance) bands. In general, temporal variations of NDVI exhibit a high correlation with the main growth stages of plants, i.e. vegetative, reproductive, maturity and senescence stages.

The combination of other spectral bands has led to the development of refined optical indices that could yield different results depending upon the type and conditions of vegetation. Studies conducted in (Haboudane et al. 2004) demonstrated that LAI values for soybean, corn and wheat can be consistently estimated from vegetation indices at different phenological stages.

Further improvements have been proposed, such as the enhanced vegetation index (EVI) which was designed in order to increase the sensitivity to vegetation changes by considering the canopy background contributions as well as the atmosphere influences (Huete et al. 2006).

Contrary to the valuable and long experience acquired in hyperspectral systems, the use of active microwave remote sensing for agriculture monitoring activities is a relatively new topic (Lopez-Sanchez and Ballester-Berman 2009), also due to the scarcity of well-established and long-term research programmes developing both air- and space-borne missions capable of providing the required radar data sets. Fortunately, this situation has entirely changed in recent years after the initiation of a large number of radar remote sensing campaigns promoted by different aerospace agencies and institutions around the world such as ESA, CSA, NASA, DLR and JAXA.

After some pioneer works on the potential of radar for characterising natural targets (see, e.g. Ulaby et al. 1987), the interest on the use of active microwave sensors was greatly stimulated during the 1990s (Henderson and Lewis 1998) as a consequence of the launch of three satellites operating at C-band, i.e. ERS, ENVISAT-ASAR and RADARSAT-1.

Among other applications, vegetation phenology monitoring has been also tackled, but the literature on this topic is still very scarce. First contributions demonstrating the relationship between radar backscatter and growth stages of rice were presented by Le Toan et al. (1989), Kurosu et al. (1995) and Le Toan et al. (1997) in 1989, 1995 and 1997, respectively. Other follow-up works to this topic were also based on the analysis of incoherent radar measurements and can be found in (Inoue et al. 2002; Chen and McNairn 2006; Koay et al. 2007; Bouvet et al. 2009). These works have shown the potential of $\mathrm{C}$-band $\mathrm{HH} / \mathrm{VV}$ ratio for rice field discrimination and that a clear intensity signature appears as a consequence of phenological development of rice plants. More recently in (Lopez-Sanchez et al. 2012a), an algorithm to estimate the phenological stages throughout the whole growing season was designed on the basis of a coherent approach. However, the phenology retrieval issue has not been explicitly studied for other crops in the literature. Indeed, the only clear example we have found on this topic is the work by Wegmüller and Werner in 1997 (Wegmüller and Werner 1997) where they described the sensitivity of interferometric coherence to the fraction of vegetation cover and the potential for tracking phenological events for corn, sugar beets, potatoes and rapeseed.

Alternatively, the interest has been focused on estimating parameters related to crop growth, such as biomass and LAI (which in turn are intrinsically related to each other), as well as crop condition and type assessment. Early contributions by 
Brown et al. (1992) and Bouman and Hoekman (1993) made use of different frequency bands for separating crop types. Also the estimation of biomass from olive groves, sunflower, corn, sorghum, rape, wheat and alfalfa was studied in (Ferrazzoli et al. 1997). Estimation of LAI with saturation effect at $2-3 \mathrm{~m}^{2} / \mathrm{m}^{2}$ has been also reported in (Ferrazzoli et al. 1992) by means of radar backscatter at C-band. The use of time series of backscattering coefficients, together with electromagnetic models and neural networks, for such a purpose has been also addressed in the literature (Del Frate et al. 2004). Backscattering coefficients at different polarimetric channels were also correlated with phenology and cultivation practices (e.g. irrigation) for several crop types in (Moran et al. 2012).

As an alternative way to retrieve information on the phenology of crops, other works have been focused on crop height estimation, given that plant height could be used as a proxy for phenology at least for certain growth stages (mainly during the vegetative phase). The applied retrieval strategy has evolved from the design of empirical relationship between coherence and height as shown in (Engdahl and Borgeaud 1998; Engdahl et al. 2001) for sugar beet, potato and winter wheat to more elaborated and robust approaches consisting of electromagnetic modelling of PolInSAR observables (Treuhaft et al. 1996; Treuhaft and Siqueira 2000; Cloude and Papathanassiou 2003) which have yielded successful results in crops such as maize and rice in laboratory conditions (Ballester-Berman et al. 2005) and maize and winter rape in the framework of an airborne campaign (Lopez-Sanchez et al. 2012b).

In summary, it can be concluded that the topic of crop phenology is an incipient research field which recently has been stimulated by the potential positive socio-economic impact that remote sensing tools offer in terms of monitoring and management tasks on crop fields at large scale.

\subsubsection{Methodology}

As a matter of fact, the problem of identifying the phenological stage of an agricultural crop can be regarded as a classification problem, where each stage corresponds to a class, and hence can be approached in a similar way to crop type mapping. This application is better suited to algorithms based on hierarchical trees or simple decision planes, since they can be tailored to match specifically the different features of the plants that change or appear as they develop. This type of rule-based algorithms facilitates the physical interpretation of the phenology retrieval procedure, since the criteria are based on scattering mechanisms (e.g. surface, dihedral, volume) and properties (e.g. extinction, depolarisation, etc.) in correspondence to the crop structure and features at each stage and in contrast with other algorithms based on the full covariance statistics (e.g. Wishart classifier). Hierarchical tree algorithms have been widely used for classification purposes in the literature and specifically for crop type mapping with PolSAR data, so they can be considered as mature since they provide consistent accuracies above $85 \%$ in crop type mapping. Therefore, hierarchical tree algorithms will be implemented and tested for this product.

The starting point is the multi-look processing or filtering of the available PolSAR images, providing the covariance or coherency matrix for each multi-looked pixel. A sliding boxcar filter has been employed since the monitored parcels are wide enough and homogeneous for applying such a filtering type. Then, all images have been geocoded. If all images were acquired with the same beam and pass, a coregistration of the whole set to a common master image could be used instead of geocoding. Once geocoded, all available PolSAR images are studied for each crop type by restricting the region of interest (ROI) to the interior of all fields of each crop. In all cases, an analysis of a large number of polarimetric observables is carried out in order to extract the most meaningful set for the crop under study. The available observables are backscatter powers and correlations (linear, Pauli and circular basis and for compact polarimetry as well), backscattering ratios for different channels and for various polarisation bases, eigenvector/eigenvalue decomposition parameters, compact polarimetry decomposition parameters and the Freeman-Durden and Touzi decomposition outputs.

This analysis is based on the representation of the observables as a function of phenology, so the reference data recorded at each acquisition date are used to define the $\mathrm{x}$-axis of their representation. For each observable, the mean and standard deviation within the parcel at every radar acquisition are obtained and plotted.

From the analysis of the evolution of all observables for each crop type, a reduced set of them will be selected for the retrieval algorithm by identifying the ones that define with more distinction particular phenological stages. Specifically, those with wider dynamic ranges and less presence of ambiguities will be chosen. Moreover, observables with easy physical interpretations will be preferred to those with less clear explanations in terms of scattering physics. With the selected set of observables, a hierarchical tree is defined by setting manually thresholds based on the previous analysis.

An important question to address for the definition of the final product concerns the required spatial resolution since phenology can be provided either at pixel level (one value per multi-looked pixel) or at parcel level (one value per parcel). In the first case, we could detect areas with different degrees of development within the same parcel, hence being also useful for localised farming practices such as irrigation and fertilisation. In the second case, we would be interested in the 
Table 3.5 Test sites and corresponding radar and validation data selected for the generation of showcases on crop phenology estimation under vegetation

\begin{tabular}{l|l|l}
\hline Application/product & Test site - radar data & Reference data \\
\hline $\begin{array}{l}\text { Crop phenology } \\
\text { estimation }\end{array}$ & Indian Head & $\begin{array}{l}\text { Intensive campaign of } \\
\text { AgriSAR2009 }\end{array}$ \\
\cline { 2 - 3 } & $\begin{array}{l}57 \text { quad-pol RADARSAT-2 images, from which 20 are used in this } \\
\text { showcase }\end{array}$ & \\
\hline
\end{tabular}

global development of the crop field, which would be considered as homogeneous.

According to the available reference data (see Appendix), we know the phenology at several ground points of each field, but (1) they are mostly coincident for the same parcel; and (2) available values are maximum and minimum values of phenological stages, instead of single values. Therefore, we have considered in the analysis that phenological data at each field and each date are the same for the whole field, being the mean the value adopted.

The inversion algorithm however has been applied at both pixel and parcel levels. This option increases the usefulness of the product for potential end-users since retrieved information is provided at different scales, i.e. at pixel level any possible heterogeneity within a field can be detected and, in addition, at parcel level an overall conclusion on the status of the field is obtained. For the estimation at parcel level, it will be computed as the mode of the estimates within the parcel in accordance with the available reference data.

\subsubsection{Experimental Results}

Test sites and corresponding radar and validation data sets selected for crop phenology estimation are summarised in Table 3.5 and further described in the Appendix.

\subsubsection{Analysis}

First we describe and justify the evolution as a function of phenology of different parameters. To this end, all parameters presenting similar evolutions are grouped. In general, from the five crop types analysed (barley, oat, wheat, field peas and canola), we have found only three main signatures, since all three cereals (barley, oat and wheat) behave similarly.

Although in principle we expected clear differences in the radar responses as a function of incidence angle (there are images acquired with angles from $22^{\circ}$ to $39^{\circ}$ ), such differences are only evident in some parameters and especially for certain crop conditions (e.g. during the vegetative phase in cereals, since ground dominates the radar response). Consequently, a joint analysis of all angles has been carried out. In some extreme situations, like two images acquired on consecutive days with $22^{\circ}$ and $39^{\circ}$, a discontinuity is expected, so this will be commented when necessary.

Finally, and according to the discussion in the previous sections, we have computed the evolutions of all parameters at pixel level after a $9 \times 9$ multi-look. The plots of the evolutions show the average and the standard deviation computed over the whole field.

In this report, we will focus our analysis on the results on cereals (which benefit most strongly from polarimetry), and some additional short comments will be given on canola and pea fields. Only observables with some trends or sensitivity to phenology will be commented for each crop type.

Sample results are shown in Fig. 3.14.

\subsection{Cereals}

Parameters with high sensitivity:

- Linear cross-polar backscatter $\left(S_{h v}\right)$ presents an increase at both the early stages (6-10 dB from stages 10 to 25-30) and the late ones (4-5 $\mathrm{dB}$ from stage 75$)^{*}$, being quite constant in the middle. Similar parameters are $S_{h h}-S_{v v}, S_{r r}$ and $S_{l l}$ backscatter and $P_{v}$ of Freeman decomposition. * Note that the increase in late stages is not present for oat (but at one single acquisition at $22^{\circ}$ ).

- HHVV correlation decreases clearly during the vegetative phase (stages 10-50) and then remains around 0.4 with important differences between acquisitions. Similar parameters:

- Average alpha increases from $0^{\circ}$ to $45^{\circ}$ only during the vegetative phase.

- $S_{r l} / S_{r r}$ and $S_{l r} / S_{l l}$ ratios show a decrease of $10 \mathrm{~dB}$ during the vegetative phase.

- Correlations RRRL and LLRL behave similarly, especially for wheat.

- Entropy shows a sudden increase from 0.2 at stage 10 to $0.7-0.8$ at stages $20-30$, and then it remains around 0.8 all the time.

- Dominant alpha $\left(\alpha_{1}\right)$ increases monotonically during the whole cycle, but it is slightly saturated after the vegetative phase (see Fig. 1.1).

- $\tau$ of Touzi decomposition is always close to zero, so the corresponding dominant alpha is like $\alpha_{1}$ from the conventional eigen-analysis.

\subsection{Canola}

The most remarkable result in this case is that the cross-polar backscatter follows a clear monotonic increasing trend for the whole growth period. This enables the phenology estimation in a straightforward way by using one single channel, HV. Indeed, coherent polarimetry does not contribute much to this crop type. 

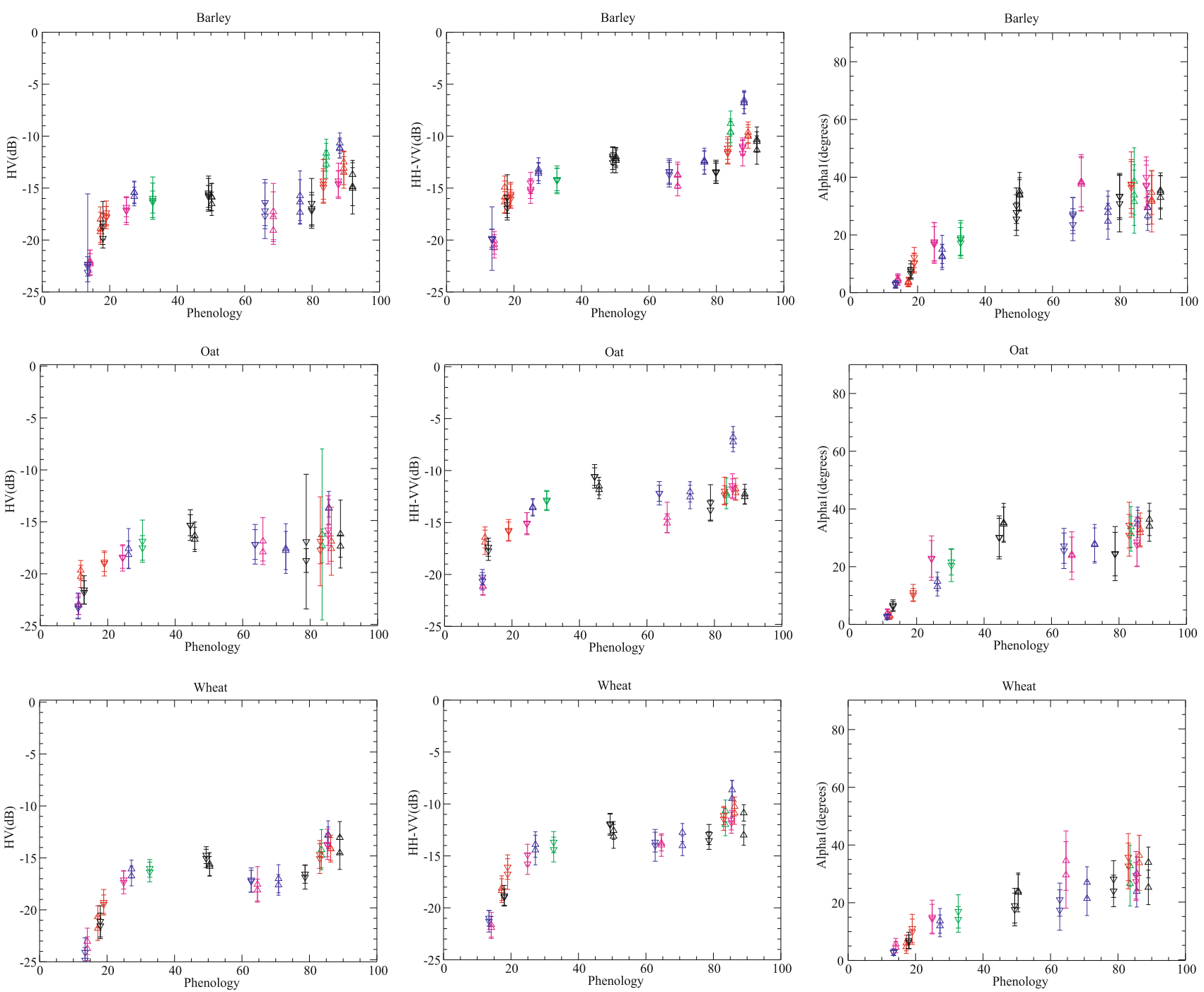

Fig. 3.14 Evolution of $\mathrm{HV}\left(t_{33}\right), \mathrm{HH}-\mathrm{VV}\left(t_{22}\right)$ and dominant alpha $\left(\alpha_{1}\right)$ as a function of phenology for barley (top row), oat (middle row) and wheat (bottom row)

\subsection{Field Pea}

In general we found that many observables here are symmetrical with respect to stages 20-25, hence making it difficult to break the ambiguity between early and late stages without any auxiliary information (e.g. time coordinate). Plants are always very short, so there is not much development or clear changes in terms of structure.

\subsubsection{Retrieval Algorithms}

In the following, details on the retrieval algorithm for cereals are given. After a close inspection of Fig. 3.14, a feasible algorithm could be designed to distinguish four different phenological intervals, i.e. early vegetative (stages 0-19), advanced vegetative (stages 20-39 or 20-44), late vegetative, reproductive and early maturation (stages from 40 or 45 to 79) and, finally, maturation (stages 80+).
The physical description of each interval is the following:

1. Surface scattering dominates the radar echo: very low entropy, alpha and alpha1 close to zero, very low $S_{h v}$ and high correlation between $S_{h h}$ and $S_{v v}$.

2. Vegetation starts to be present in the radar response, hence increasing entropy. Dominant alpha $\left(\alpha_{1}\right)$ is low (less than $20^{\circ}$ ), but alpha has already reached $40^{\circ}$. Backscatter power will remain quite constant at all channels during this stage and the next one.

3. This corresponds to a moment of fast development in terms of phenology (buds, flowers, etc.), but not much change in terms of structure and, thus, radar response at least at C-band. Backscatter powers remain constant and both alpha and alpha1 too. 
4. Finally, backscatter power increases at $S_{h v}$ and $S_{h h}-S_{v v}$ channels as a result of an increase of the randomness of the structure of the plants (but for oat, which remains as in previous stages since its morphology does not change).

A basic hierarchical tree algorithm can be defined in terms of just two parameters: dominant alpha angle $\left(\alpha_{1}\right)$ and backscatter power at $S_{h v}$ or $S_{h h}-S_{v v}$ ( $t_{33}$ or $t_{22}$ entries of the coherency matrix). The proposed algorithm is depicted in Fig. 3.15.

\subsubsection{Results and Validation}

The results obtained by applying the algorithm proposed in the previous section are analysed here for each crop type separately. As mentioned above, we will concentrate in cereals where the benefits of polarimetry are well evident. A figure showing the output of the retrieval algorithm applied at pixel level for wheat fields and for the 20 images will be presented. The statistics of the retrieved values and their comparison against the reference data will be also shown in form of a table. Results on oat and barley are just summarised in the text.

\subsection{Wheat}

Figure 3.16 shows the retrieved results for wheat. We can appreciate how the most frequent phenology value at each data is in perfect agreement with the reference data at all dates but for one image acquired on 2nd of July (with $22^{\circ}$ incidence). This acquisition corresponds to an extreme incidence angle, so the proposed algorithm (common for all incidences) is more likely to fail. Nevertheless, the first images provide a $100 \%$ of pixels with the right value. In later acquisitions, the transitions between successive stages are, in general, quite smooth in terms of the amount of pixels estimated to be at each stage around the transitions.

For some images there is a non-negligible amount of pixels (more than 25\%) assigned to wrong stages. These cases correspond to either dates of transition between successive stages or cases where the particular incidence angle affects more clearly the radar response. Anyway, the overall result demonstrates that the proposed algorithm is quite reliable despite its simplicity and it provides right estimates for 19 of the 20 images.

\subsection{Oat}

Results for oat (not shown here) are not as good as for wheat after the early vegetative phase. In this case, the radar response does not change significantly from the sixth acquisition date onwards. Consequently, it is virtually impossible to distinguish the two last intervals, from stage 45 to the end of the season, and also separating the advance vegetative (interval 2) from the later stages. With the proposed approach, the most frequent value of retrieved phenology from the sixth to the last image corresponds to interval 3 but in five of the images, hence demonstrating the lack of sensitivity for this crop type. The overall validation provides 13 right estimates at parcel scale from the 20 cases.

Attending at the physical characteristics of oat, the vegetation volume, it is less dense and tall than other cereals (e.g. wheat and barley), so the radar response does not exhibit clear variations after the end of the vegetative phase, and, moreover, the ground contribution is more present than for other cereals.

\subsection{Barley}

The overall performance of the proposed algorithm for barley (tables not shown here) is quite similar to that of wheat, so the same comments apply. In this case the algorithm provides right estimates in all 20 images.

\subsubsection{Comparison with Single- $/$ Dual-Polarisation Data}

The analysis of the evolutions of radar observables as a function of phenology suggests that the dimensionality of the polarimetric space influences the number of different stages that could be identified for each crop type. As in the previous lines, the following analysis is focused on cereals since in this case polarimetry does play a substantial role for monitoring purposes.

For single polarisation, $\mathrm{HH}$ and VV exhibit low sensitivity to phenology and large dependence on incidence angle and even on ascending/descending mode (probably due to row orientation w.r.t. radar) in early stages. HV shows three stages in its evolution as a function of phenology, i.e. initial fast increase at early vegetative, slow decrease at central part
Fig. 3.15 Basic hierarchical tree algorithm for cereals. $t_{22}$ can be equivalently substituted by $t_{33}$ setting the threshold in $-15 \mathrm{~dB}$

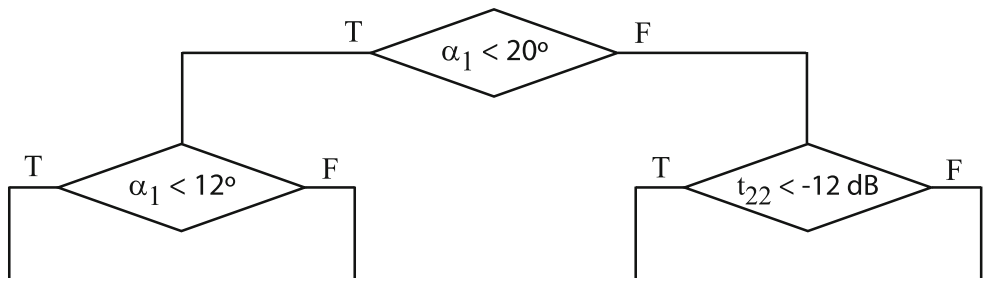

1. Early vegetative (stages 0-19)
2. Advanced vegetative (stages 20-44)
3. Reproductive

(stages 45-79)
4. Maturation (stages $80+$ ) 

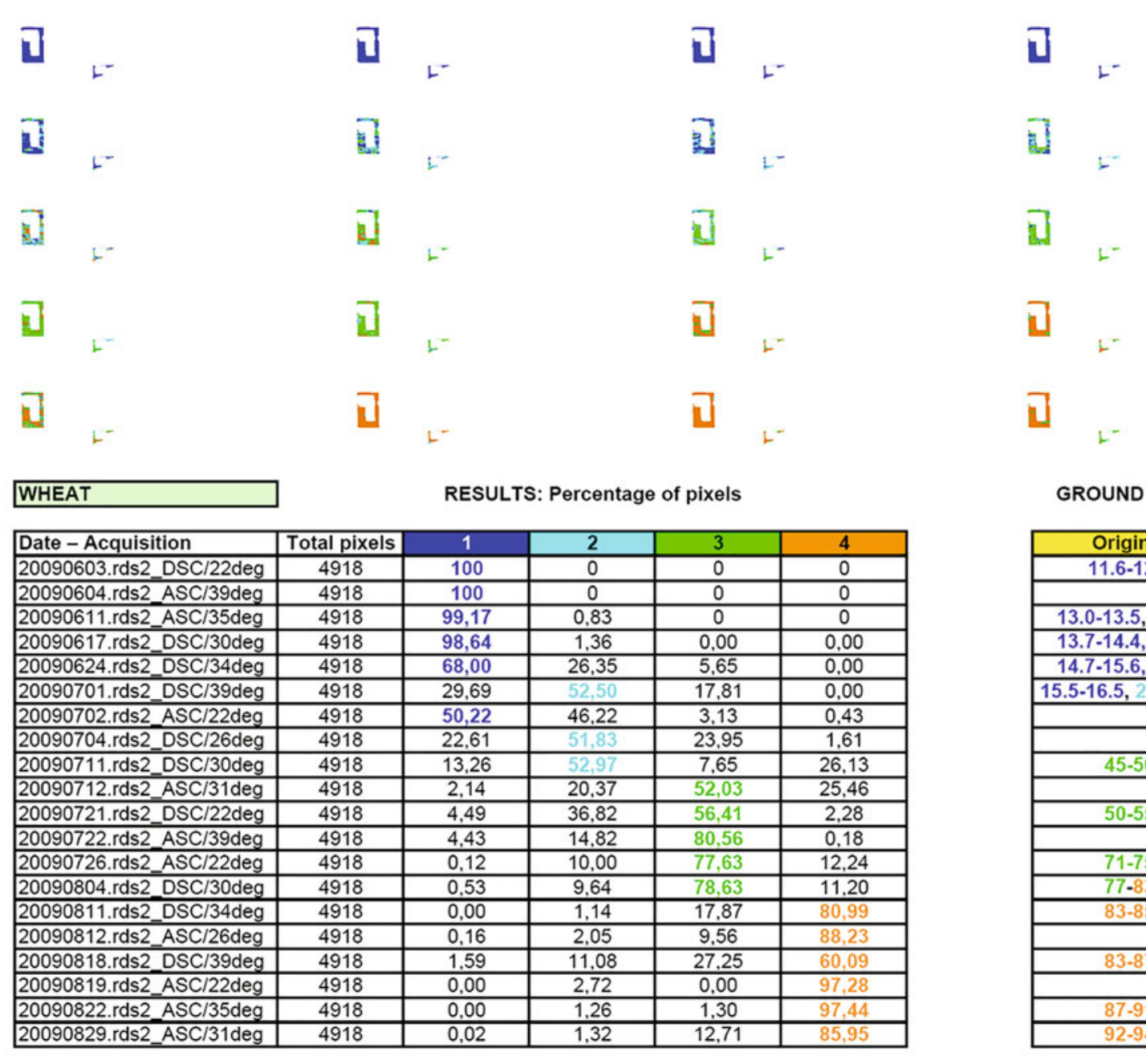

GROUND DATA

\begin{tabular}{|c|c|}
\hline Original & Acq. Date \\
\hline $11.6-12.3$ & 20090601 \\
\hline & \\
\hline $13.0-13.5,20-22$ & 20090610 \\
\hline $13.7-14.4,20-22$ & 20090617 \\
\hline $14.7-15.6,20-24$ & 20090624 \\
\hline $15.5-16.5,21-24,31$ & 20090630 \\
\hline & \\
\hline & \\
\hline $45-50$ & 20090708 \\
\hline & \\
\hline $50-55$ & 20090715 \\
\hline & \\
\hline $71-75$ & 20090724 \\
\hline $77-83$ & 20090730 \\
\hline $83-85$ & 20090806 \\
\hline & \\
\hline $83-87$ & 20090813 \\
\hline & \\
\hline $87-91$ & 20090821 \\
\hline $92-94$ & 20090829 \\
\hline & \\
\hline &
\end{tabular}

Fig. 3.16 Results obtained for wheat: Percentage of pixels assigned to each stage at each image and available reference data. The most frequent value at each date is coloured according to the scale employed in the map

from 20 to 80 stages and late fast increase. Therefore, the number of stages to be separated is smaller than with full-pol, and there is more uncertainty between stages $10-20$ and $20+$. Moreover, the same threshold would not fit equally all cereals and should be adapted to each type.

In case of dual-pol, and provided the mentioned lack of sensitivity of $S_{h h}$ and $S_{v v}$, typical dual-pol systems gathering $\left[S_{h h}, S_{v h}\right]$ or $\left[S_{v v}, S_{h v}\right]$ data do not show a clear improvement with respect to $S_{h v}$. Anyway, the joint use of $S_{h v}$ and any of the $S_{h v} / S_{v v}$ or $S_{h v} / S_{h h}$ ratios provides enough information for barley and wheat to classify correctly the phenology for the three intervals mentioned in the previous paragraph (i.e. stages below 20 , from 20 to 80 and above 80 ). Intermediate stages, however, are not separable in this observation space.

A $\left[S_{h h}, S_{v v}\right]$ coherent measurement, instead, provides similar performance to full-pol, since $\alpha_{1}$ is quite similar to the $\alpha_{1}$ gathered with full polarimetry and $S_{h h}-S_{v v}$ is already used by the proposed algorithm. Anyway, such acquisitions suffer the same drawback of full polarimetry in terms of spatial coverage, due to the reduced swath required by doubling the PRF of the radar system.

Compact polarimetry (Charbonneau et al. 2010; BallesterBerman and Lopez-Sanchez 2012; Cloude et al. 2012) is expected to offer a slightly lower performance than full polarimetry but with a wider swath capability may suffice in some applications. Note that $t_{33}, t_{22}$ and $\alpha_{1}$, used for cereals in this study, are mapped approximately in an equivalent way by compact-pol using $P_{v}, P_{d}$ and $\alpha_{s}$.

\subsubsection{Discussion on Role of Polarimetry, on the Maturity of the Application and Conclusions}

Figure 3.17 summarises the useful parameters for each crop type according to the retrieval results presented previously. We confirm that the sensitivity of C-band polarimetry to crop 


\begin{tabular}{ll}
\hline Crop type & Useful observables \\
\hline Barley & \\
Oat & $\begin{array}{l}\alpha_{1}, S_{h h^{-}} S_{v v}\left(t_{22}\right), S_{h v}\left(t_{33}\right), P_{v} \text { of Freeman decomp., } S_{r r}, S_{l l}, S_{r l} / S_{r r}, S_{r l} / S_{l l}, \text { cor- } \\
\text { relations: HHVV, RRRL and LLRR }\end{array}$ \\
Wheat & \\
Canola & $S_{h h^{-}} S_{v v}\left(t_{22}\right), S_{h v}\left(t_{33}\right), P_{v}$ of Freeman decomp., $S_{r r}, S_{l l}$ \\
Pea & $\begin{array}{l}S_{h h^{-}} S_{v v}\left(t_{22}\right), \text { Std.Dev. }\left\{S_{r r}\right\}, \text { HHVV correlation, entropy, average alpha, } P_{v} \\
\text { of Freeman decomp., } S_{r r}, S_{l l}, S_{r l} / S_{r r}, S_{r l} / S_{l l}\end{array}$ \\
\hline
\end{tabular}

FULL POLARIMETRY

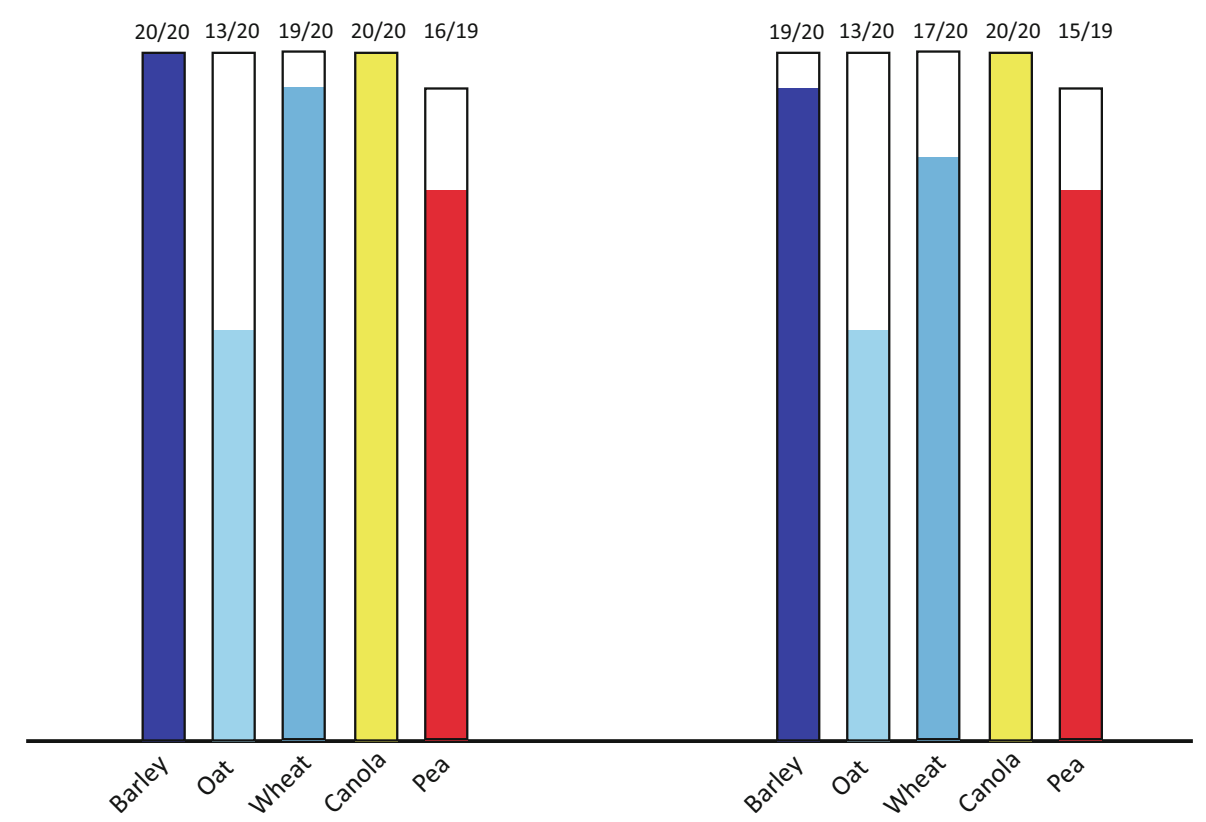

COMPACT POLARIMETRY

Fig. 3.17 Top: summary of useful parameters for each crop type. Bottom: overall performance

phenology is defined by the presence of different morphologies of plants and parcels as they develop along the cultivation cycle. For cereals with distinct plant structures at different stages, polarimetry enables the estimation of their growth stage (from a set of 4 significant intervals) by exploiting just a single radar acquisition, without any other additional information. It is also pointed out that the use of time coordinate, enabled by the availability of time series of radar data from current SAR sensors, will definitely improve the estimation accuracy even more.

The conclusions from this study can be extrapolated to other crop types by taking into account the physical rationale employed to establish the retrieval algorithms. Hence, phenology is likely to be retrieved for every crop with development features analogous to those analysed here. In addition, the wide range of incidence angles employed in this study, despite their influence in the observables, demonstrates the robustness of this application.

According to the most important observables found in this study $\left(t_{33}, t_{22}\right.$ and $\left.\alpha_{1}\right)$, a compact-pol sensor would be able to provide most of the polarimetric sensitivity required for this application and wide swath coverage. It is noted that only in case of canola fields, because of its particular morphology, dual-pol and single-pol systems would suffice.

It should be noted that all approaches validated in this study make use of backscattering coefficients (echo power) at some stages, which may limit its applicability in case of radiometric fluctuations due to diverse causes. In this sense, a phenology retrieval algorithm based on amplitudeindependent polarimetric observables has been demonstrated for rice fields at X-band (Lopez-Sanchez et al. 2012a) and C-band (Lopez-Sanchez et al. 2014). 


\subsection{Wetland Observation}

\subsubsection{C-Band Polarimetric Time Series for Delineating and Monitoring Seasonal Dynamics of Wetlands}

\subsubsection{Introduction, Motivation and Literature Review}

Land use changes, especially in agricultural landscapes, are considered as the major cause of negative environmental impacts, which can be aggravated by climate change. Drainage of wetlands for land reclamation and extraction of groundwater to irrigate agricultural land have caused degradation of soil and water quality and biodiversity loss in some parts of the world. Wetlands are complex ecosystems, permanently or seasonally flooded that provide many ecological, biological and hydrologic functions (Hubert-Moy 2006); this natural resource plays an important role in the regulation of water flow, in the protection of the water quality and in the sustainable management of ecosystems (Maltby 2009). Damaged in the past because considered as unsanitary and unfarmable areas, this ecotone or transition area is a fragile and threatened ecosystem. Preventing the wetland reduction and degradation has now become a priority. Therefore, both inventory and characterisation of wetlands constitute an important stake from an environmental but also socioeconomical point of view. A recent study proposed a method to inventory wetlands based on the functional analysis of potential, existing and effective wetlands (Mérot et al. 2006). The authors have shown that it is important to localise existing and potential wetlands because the area between them can be considered as negotiation areas for restoration purposes. It has also been demonstrated that the length of the contact between existing wetlands and the dry land - including potential wetlands - plays an important role in the denitrification process. For these reasons, a precise determination of the limits of existing and potential wetlands is an important environmental issue. Moreover, little attention has been devoted to our knowledge to the evaluation of wetlands functionalities (for hydrologic processes but also for bio-geochemical and ecological functions) on large areas. In these last years, intensive research efforts have focused on the identification and broad delineation of wetlands (Technical Report Y-87-1 1987). Remotely sensed data are currently used to identify, delineate and characterise wetlands (Ozesmi and Bauer 2002; Hubert-Moy et al. 2006). Optical data provide interesting information to inventory vegetation and agricultural practices in wetlands but are limited to cloud-free periods. For these reasons it is not possible to precisely delineate saturated areas extent as well as water cycles and water levels in these areas with passive remote sensing techniques. Although radar is not sensitive to visibility conditions and can be obtained day or night and through smoke and cloud cover, the spatial resolution of radar imagery that has been used until now was too low to investigate wetlands with a sufficient level of precision. For this purpose, quad-polarimetric and fine-resolution SAR data sets show great potential for mapping wetlands. The objective of this showcase is to address the issue of evaluating fully polarimetric RADARSAT-2 time series data sets for monitoring the seasonal dynamics of saturated areas extent in wetlands. To that end, the development and validation of a general PolSAR segmentation including multitemporal analysis of wetland evolution and polarimetric decomposition are presented.

One fundamental task in wetland monitoring is the regular mapping of temporarily flooded areas. Monitoring and mapping wetlands on a large scale is becoming increasingly more important, and satellite remote sensing provides today a practical approach. Remotely sensed data are widely used to identify, delineate and characterise wetlands. Optical data provide interesting information of improved spectral characterisation of vegetation and soil types to inventory vegetation and agricultural practices in wetlands but are limited to cloud-free periods. For this reason it is not possible to precisely delineate saturated areas extent as well as water cycles and water levels in these areas with passive remote sensing techniques. Due to the independence of weather and illumination conditions, SAR sensors could provide a suitable data base and have been proved today to be an effective tool offering great potential for quantitative monitoring and mapping of wetlands.

The topic of wetland monitoring by means of SAR remote sensing has been mostly addressed in the past by analysing temporal variations of the backscattering coefficient. The use of single-polarisation SAR data has been shown to be important for wetland water extent characterisation. However, single-polarisation SAR in vegetation-type discrimination which is necessary for wetland mapping presents a limited capability. A good overview of SAR wetland monitoring is given in (Bourgeau-Chavez et al. 2009) and (Lang and Kasischke 2008). A multi-polarised attempt is reported in (Henderson and Lewis 2008) where the amplitudes in horizontal and vertical polarisation, but no phase information, are interpreted and correlated with certain wetland characteristics.

Until today just a few publications are concerned with the use of fully polarimetric SAR data sets for wetland monitoring. The operational use of polarimetric SAR is not yet really demonstrated and is still at a study level. The results shown in different publications are always at a preliminary result stage and had never been confirmed when applied on other test sites or under different acquisition configurations (multifrequency or multi-angular). The most important publications 
in that topic are mainly based on a change detection analysis or trying to show that there exist relationships between some specific polarisation parameter and the related scattering mechanism type.

In (Liao and Wang 2010), fully polarimetric RADARSAT-2 data sets are investigated for wetland classification. The target decomposition is used for optimum characterisation of wetland target scattering, and it is shown that the polarimetric information permits the discrimination of eight classes of land surface, based on the use of the entropy and alpha parameters, and leads to an effective classification of two wetland classes: closed and open.

In (Park et al. 2010), an effective method of extracting geophysical information of tidal wetlands (zone of interaction between marine and terrestrial environments ) is proposed, based on the use of fully polarimetric forward/inverse scattering models for quantitative estimation of intertidal mudflats including surface geometric characteristics such as the roughness of the scattering surface.

In (Koch et al. 2012) multi-frequency (RADARSAT2 and ALOS-PALSAR) analysis is conducted for characterising and mapping wetland conditions in a semiarid environment in Central Spain. The results suggest that the fully polarimetric SAR data enables a better separation of the vegetation structure and fragmentation than with the optical data.

In (Brisco et al. 2011), (Schmitt et al. 2012) and (Schmitt and Brisco 2013), three polarimetric decomposition techniques (Cloude-Pottier, Freeman-Durden and normalised Kennaugh elements) are compared to each other in terms of identifying the wetland flooding extent as well as its temporal change. The image comparison along the time series is performed with the help of a novel curvelet-based change detection method for the enhancement of polarimetric decomposition channels, as well as temporal differences in these channels. The results indicate that the decomposition algorithm has a strong impact on the robustness and reliability of the change detection.

In (Patel et al. 2009), scattering models based on physical principles have been applied to characterise the wetland targets like open water habitat and various types of aquatic vegetation with or without standing water, along with various species of forested areas. Entropy, alpha angle and anisotropy have been derived, thus enabling to understand the differences in wetland targets in terms of their scattering behaviour at the L- and P-bands. A significant outcome of this study is that it explores and demonstrates the potential of the state-of-the-art technique of polarimetric SAR for characterising scattering behaviour of various components of a wetland ecosystem.

In (Yajima et al. 2008) is proposed an analysis of the seasonal changes of a wetland by a modified polarimetric four-component scattering power decomposition method. It is shown by the modified scheme that the seasonal changes and features of the vegetation of a lagoon can be clearly detected and observed clearly.

At last, in (Touzi and Gosselin 2010), the TSVM decomposition is investigated when applied for wetland monitoring. Different polarimetric decompositions are studied and compared, and some new polarimetric parameters are extracted that characterise uniquely the scattering type with a complex entity, whose both magnitude and phase have been shown very promising for wetland class characterisation and peatland characterisation. It has been shown, for example, that the scattering type phase permits the detection of water flow variations beneath the peat surface.

Until today no publications are concerned with the use of fully polarimetric SAR series data sets to identify and locate the seasonal dynamics of saturated areas in wetlands. To that end, the development and validation of a supervised PolSAR segmentation including multitemporal analysis of wetland evolution and polarimetric decomposition method are presented in this showcase. The proposed methodology is based on the segmentation of a polarimetric descriptor, the Shannon entropy, which has been shown to be a very sensitive parameter to the temporal variability of flooded areas.

\subsubsection{Methodology}

The employed methodology is presented on Fig. 3.18. The RADARSAT-2 quad-polarimetric SAR image pre-processing and processing has been conducted using the PolSARpro software. The first step consists in extracting the polarimetric $3 \times 3$ T3 coherency matrix which is independent of the polarimetric absolute phase, in order to apply the geocoding process directly on the elements of the T3 matrix. The geocoding of the RADARSAT-2 quad-polarimetric slantrange SAR images to the geographic ground range coordinate system is performed using 46 precise ground control points, uniformly distributed over the subset image, selected from the ortho-rectified ALOS-PRISM and ALOS-AVNIR-2 images used as reference images (Marechal et al. 2010). Due to the fact that the topography of the investigated area is relatively flat, the three inherent and specific geometrical distortion sources in SAR images (foreshortening, layover and radar shadow) do not occur in such a case, and one can conclude that the polarimetric data quality is preserved after the geocoding process (Toutin et al. 2013). To validate the rectification procedure, corner reflectors have been set in the investigated area during the RADARSAT-2 acquisitions. As these corner reflectors have been precisely located with a differential GPS, it has been possible to assess the geocoding accuracy, thus leading to an RMSE error lower than 1 pixel (Marechal et al. 2012).

Concerning the RADARSAT-2 quad-pol SAR image processing, different polarimetric decomposition methods (Huynen, Cloude, Barmes, Holm, Freeman, Van Zyl, 


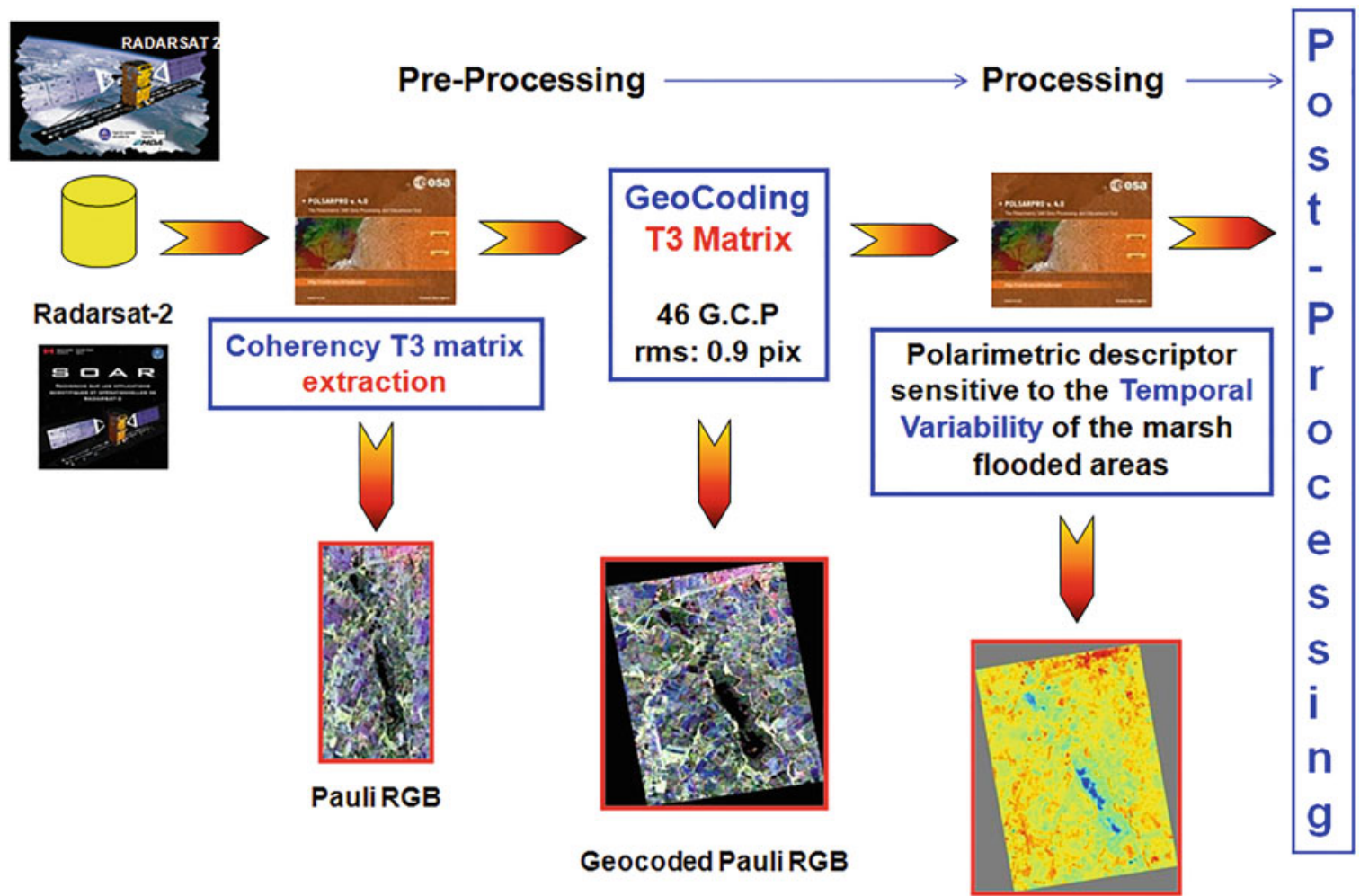

Fig. 3.18 Proposed methodology

Yamaguchi, Cloude-Pottier and Shannon entropy decompositions) (Lee and Pottier 2009) or specific PolSAR image analysis when applied on wetland characterisation and interpretation (Yajima et al. 2008; Touzi and Gosselin 2010) has been investigated in order to extract one polarimetric descriptor that could be very sensitive to the temporal variability of the marsh flooded areas. The temporal coefficient of variation along the 15 RADARSAT-2 quad-polarimetric SAR images has been derived for each of the polarimetric parameters extracted from the polarimetric decompositions, and among all of them, the Shannon entropy (SE) (Lee and Pottier 2009), defined in equation (1.171), has shown the most pronounced contrast between the marsh flooded areas and the surrounding, allowing a segmentation of the data to extract only the water table.

Figure 3.19 shows the normalised Shannon entropy (SE) parameter when applied on the Pleine-Fougères PolSAR image. It is important to point out that the investigated area is a permanent windy area and the open water area becomes a slightly rough surface. Consequently and despite the quite low intensity level, the backscattering mechanism corresponds to a single-bounce scattering mechanism with an associated Shannon entropy low value. Figure 3.20 shows the temporal coefficient of variation $\operatorname{CV}(x)=\frac{\sqrt{\operatorname{var}(x)}}{E\{x\}}$ of the Shannon entropy parameter over the 1-year time series. It can then be seen that this polarimetric descriptor is a very sensitive parameter to the temporal variability of the marsh flooded area.

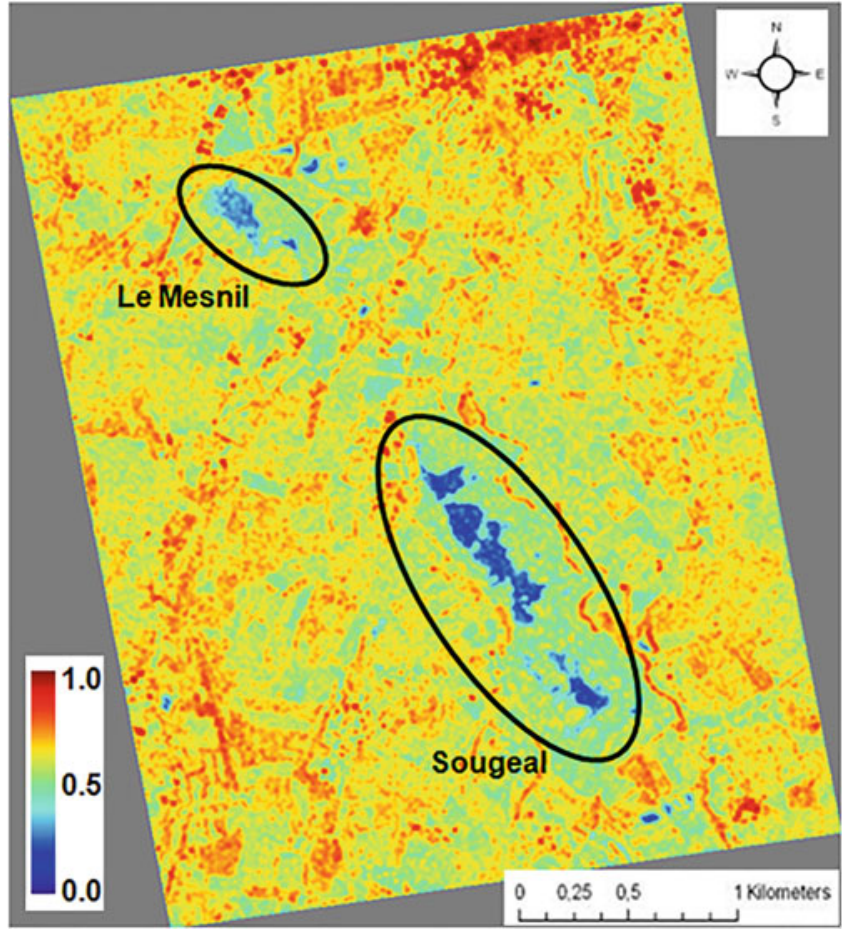

Fig. 3.19 The normalised Shannon entropy (SE) image

The original concept proposed in this methodology is based on the post-processing which is based on a segmentation of the Shannon entropy image in order to detect and 
extract the limits of the open water area and its evolution during the time period under study. Indeed, the extent of the flooding area and its timing exert a strong influence on ecological patterns such as the distribution and diversity as well as the amount of plant community in the wetland (Bourgeau-Chavez et al. 2009). The segmentation-based image procedure that has been used is based on the following steps: calculation of gradient values map, computation of global statistics (cumulative relative histogram) and application of watershed transform on the modified gradient map to detect homogeneity sets of pixels. This segmentation-based

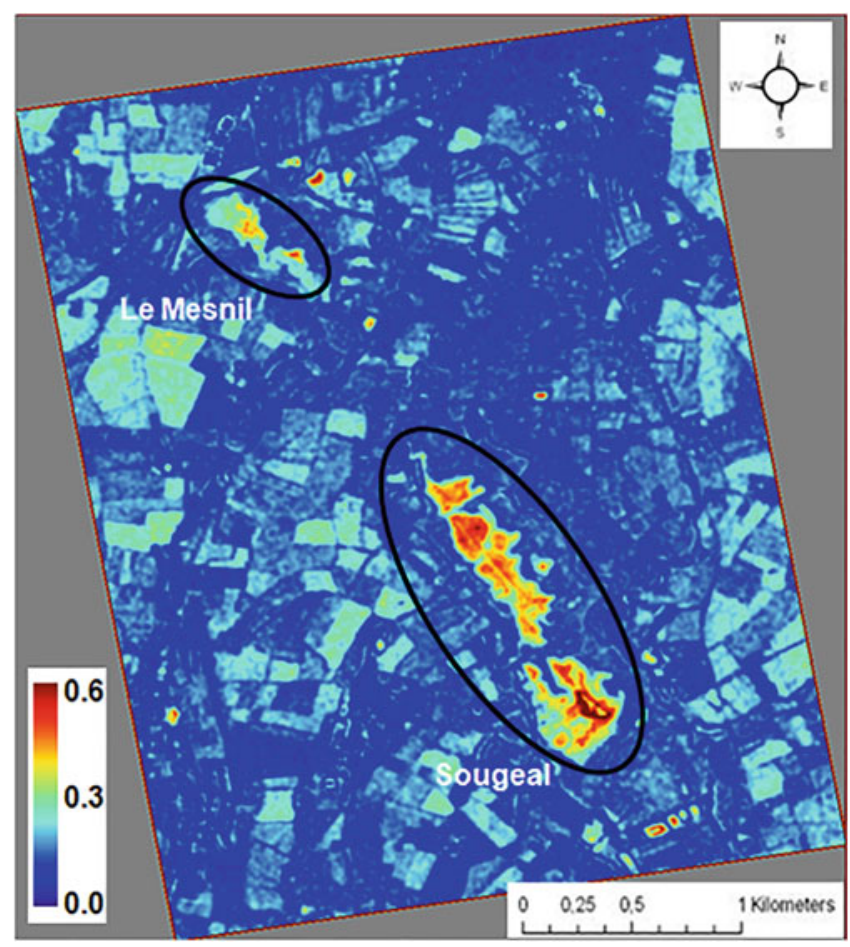

Fig. 3.20 Temporal coefficient of variation of the Shannon entropy parameter image processing system is implemented in ENVI software (ITT Visual Information Solutions 2007, Boulder, USA), and details on this patented application can be found in (Xiaoying 2009).

The Shannon entropy image has been segmented to identify open water and saturated areas. For this, segmentation parameters, like Shannon entropy threshold values, pixel population and neighbourhood limit, have been set up from the first RADARSAT-2 quad-pol SAR image acquired on 22nd of February 2010. Due to both the pixel size $\left(5 \times 6 \mathrm{~m}^{2}\right)$ of the SAR image, versus the area of the investigated study site, and the ground truth observations, a good compromise in the setting method has been to fix the pixel population to 30 for open water to 40 for saturated area and to fix the neighbourhood limit to 8 pixels in both cases. The threshold used during the segmentation procedure has been set up from a histogram analysis of the Shannon entropy value distribution and has been fixed to 0.4 to separate the open water case to the saturated area case as shown in Fig. 3.21. Once trained on this first image, the same resulting and fixed parameters have been applied for the segmentation of all the 14 other SAR images, without applying any new training on each image.

\subsubsection{Experimental Results}

Test sites and corresponding radar and validation data sets selected for wetland delineation and monitoring are summarised in Table 3.6 and further described in the Appendix.

The segmented image corresponding to the first RADARSAT-2 quad-pol SAR image acquired on 22nd of February 2010 is shown in Fig. 3.22. The validation of the segmentation of the Shannon entropy ( $S E)$ image has been conducted using the methodology presented in Sect. 3.5.1.2. On Fig. 3.23 are displayed the results of the segmentations corresponding to the maximum (2010/12/31) and minimum (summer acquisitions from 2010/07/16 to 2010/09/26)
Fig. 3.21 Histogram analysis of the Shannon entropy value distribution and corresponding thresholds

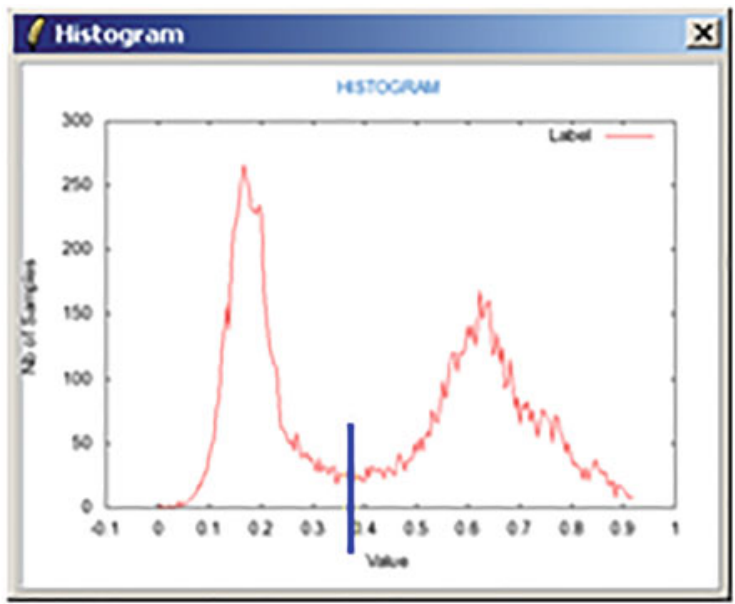


Table 3.6 Test sites and corresponding radar and validation data selected for the generation of showcases on wetland delineation and monitoring

\begin{tabular}{l|l|l}
\hline Application/product & Test site - radar data & Reference data \\
\hline $\begin{array}{l}\text { Wetland delineation } \\
\text { and monitoring }\end{array}$ & Pleine-Fougères, Brittany, France & $\begin{array}{l}\text { ALOS-PRISM } \\
(16 / 03 / 2010)\end{array}$ \\
\cline { 2 - 3 } & 16 RADARSAT-2 Fine Quad-Pol (FQ23) & $\begin{array}{l}\text { ALOS-AVNIR- } \\
2(16 / 03 / 2010)\end{array}$ \\
\cline { 2 - 4 } & $\begin{array}{l}22 / 02 / 2010,16 / 03 / 2010,11 / 04 / 2010,05 / 05 / 2010,29 / 05 / 2010,22 / 06 / 2010,16 / 07 / 2010,09 / 08 / \\
\text { Lidar }(05 / 04 / 2009)\end{array}$ \\
\cline { 2 - 4 } & $2010,02 / 09 / 2010,26 / 09 / 2010,20 / 10 / 2010,13 / 11 / 2010,07 / 12 / 2010,31 / 12 / 2010,24 / 01 / 2011$, & $0.5 \mathrm{~m} \mathrm{DTM}$ \\
\cline { 2 - 4 } & $17 / 02 / 2011$ & Ground truth data \\
\hline
\end{tabular}
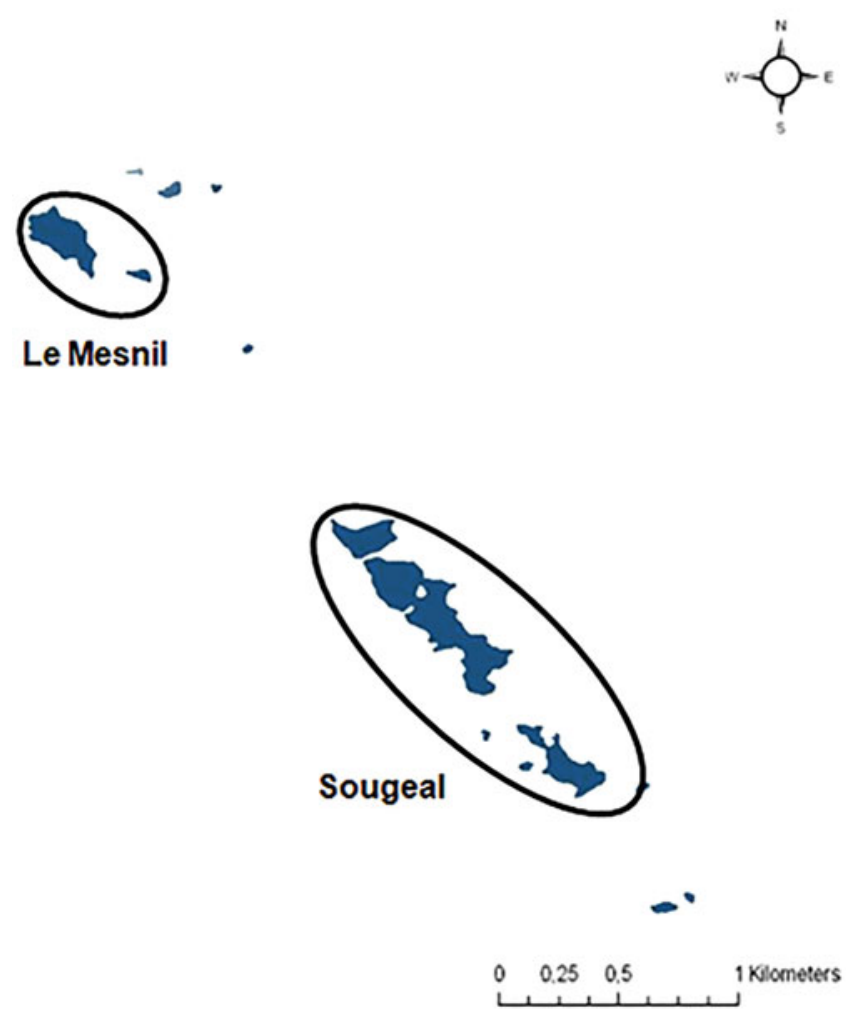

Fig. 3.22 The segmented Shannon entropy (SE) image with the open water in blue

extension of the water table. The segmentation result for the acquisition of March (2010/03/18) is also shown as a classical example. The measurement stations, located along the transect, are represented with the symbol $\boldsymbol{O}$, and the symbol t represents the reference fix point. The detected limits of the water table for the three examples are represented with the symbols (December), $\boldsymbol{X}$ (March) and $\boldsymbol{*}$ (summer). As it can be seen, there exists a prefect correspondence between the results of the segmentation and the limit of the water table detected during the different ground truth campaigns.

More of that, to assess the results, the spatial distribution of the open water, extracted from the segmented image, is displayed over the lidar image. The analysis of the altimetric profile, obtained from the digital elevation model, confirms the shape of the limit of the open water according to the local microtopography (presence of little canal with deposits or presence of two holes, artificial depression on the ground, etc.).

This 1-year time series of segmented Shannon entropy images is merged in a geographic information system in order to create a precise map of permanent and temporary water. Figure 3.24 provides an overview of the variations of the water table over the wetland site from February 2010 to February 2011, in showing the minimum and maximum water extent of the water table, including open water bodies and inundated vegetation and soils, during one hydrological year.

\subsubsection{Comparison with Single-I Dual-Polarisation Data}

The fully polarimetric RADARSAT-2 data of wetlands are used to simulate dual-polarimetric mode data from the upcoming RADARSAT Constellation Mission (CSA) and Sentinel-1 (ESA). The simulated dual-polarimetric SAR data are then used to evaluate the Shannon entropy for wetland monitoring, and the results are compared with the fully polarimetric data. In the dual-polarimetric case, the two terms of the Shannon entropy are given by:

$$
\begin{aligned}
& \mathrm{SE}_{I}=2 \log \left(\frac{\pi e I_{T}}{2}\right)=2 \log \left(\frac{\pi e \operatorname{trace}\left(\mathbf{C}_{2}\right)}{2}\right) \\
& \mathrm{SE}_{P}=\log \left(1-p_{T}^{2}\right)=\log \left(4 \frac{\left|\mathbf{C}_{2}\right|}{\operatorname{trace}\left(\mathbf{C}_{2}\right)^{2}}\right)
\end{aligned}
$$

where $\mathbf{C}_{2}$ stands for the $2 \times 2$ covariance matrix of one of the possible dual-polarimetric target vector: $\left[S_{h h}, S_{v h}\right]^{T},\left[S_{h v}\right.$, $\left.S_{v v}\right]^{T}$ or $\left[S_{h h}, S_{v v}\right]^{T}$.

Figures 3.25 and 3.26 show the normalised Shannon entropy (SE) parameter when derived from the dualpolarimetric target vectors $\left[S_{h h}, S_{v h}\right]^{T}$ and $\left[S_{h v}, S_{v v}\right]^{T}$.

As it can be seen, the results are consistent for both dualpolarisation Shannon entropy and the fully polarimetric one (Fig. 3.19). This result is very interesting and mainly promising in the sense that the methodology developed for the fully polarimetric case can be applied without any restriction to the dual-polarimetric case as the input feature seems to be the same. Delineating and monitoring the seasonal 
Fig. 3.23 Validation of the limits of the water table extracted from the segmented images with the limits detected during the corresponding ground truth campaigns

Fig. 3.24 Variations of the water table over the wetland site from February 2010 to February 2011
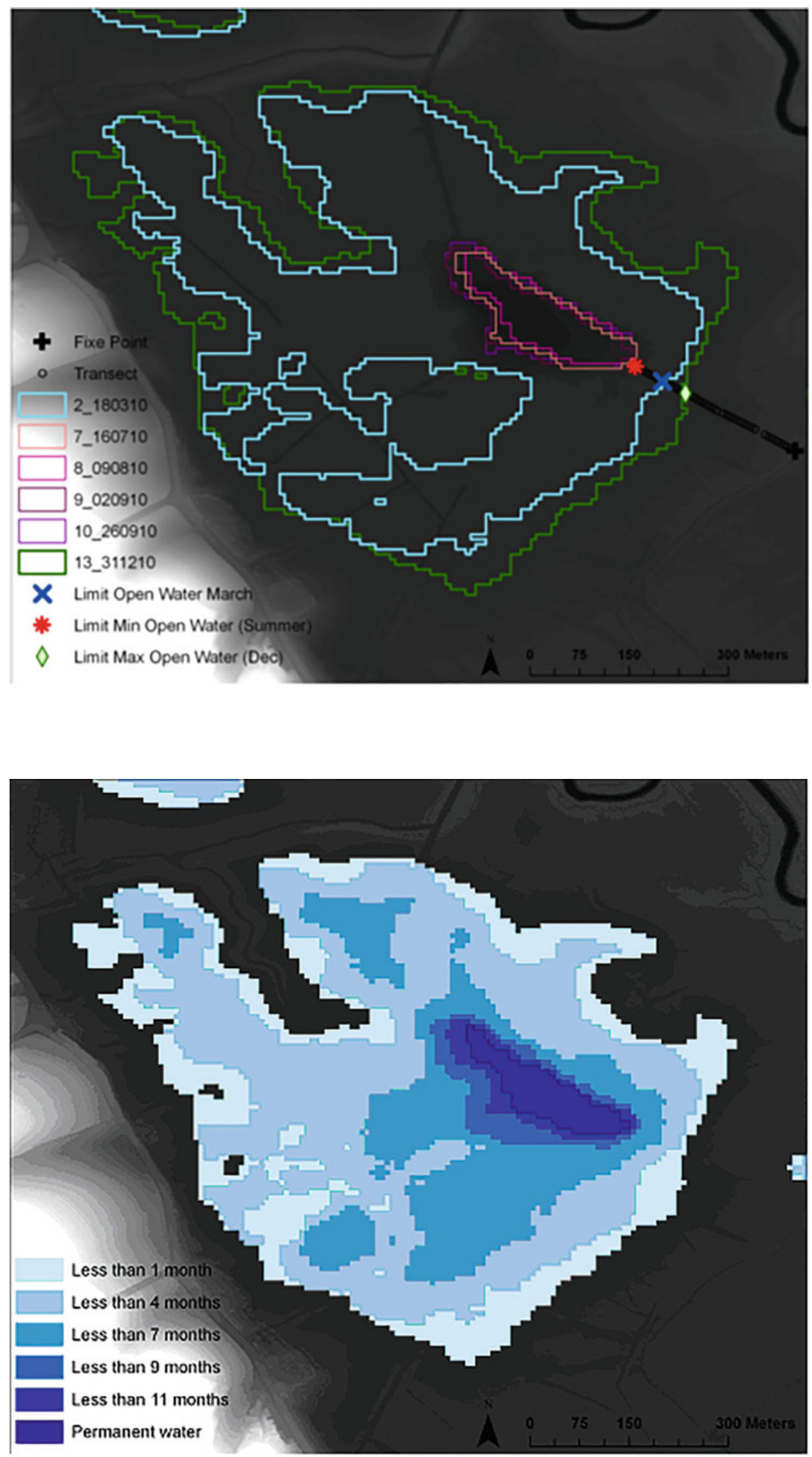


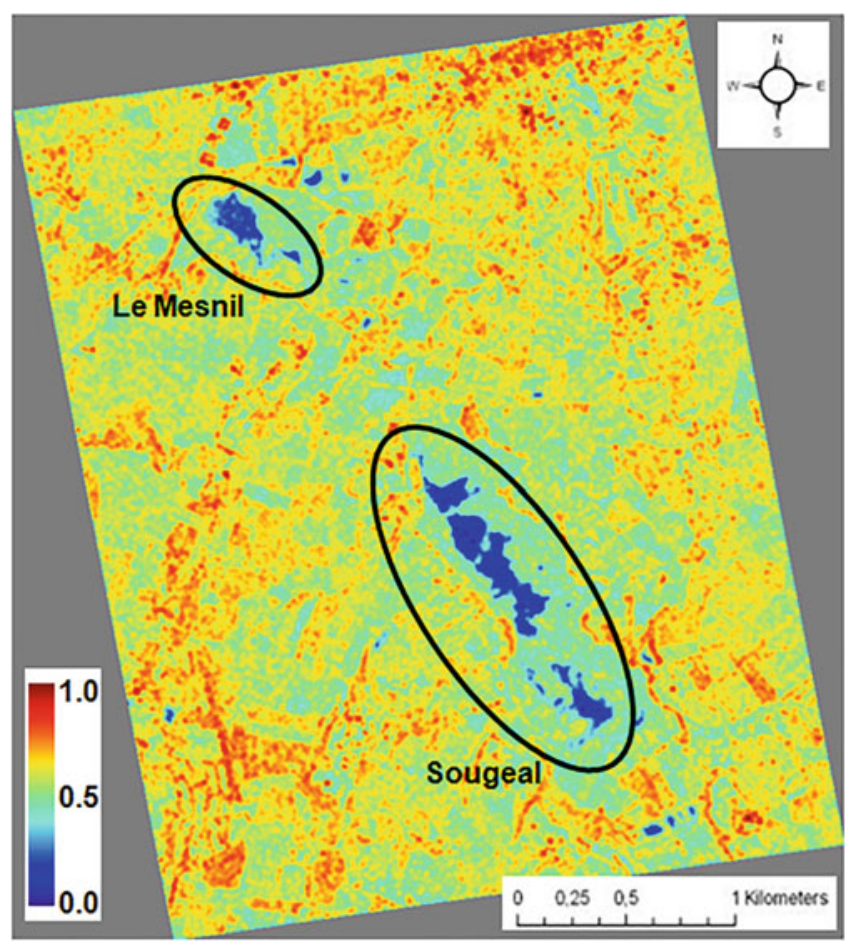

Fig. 3.25 The normalised Shannon entropy (SE) image in the case of the target vector $\left[S_{h h}, S_{v h}\right]^{T}$

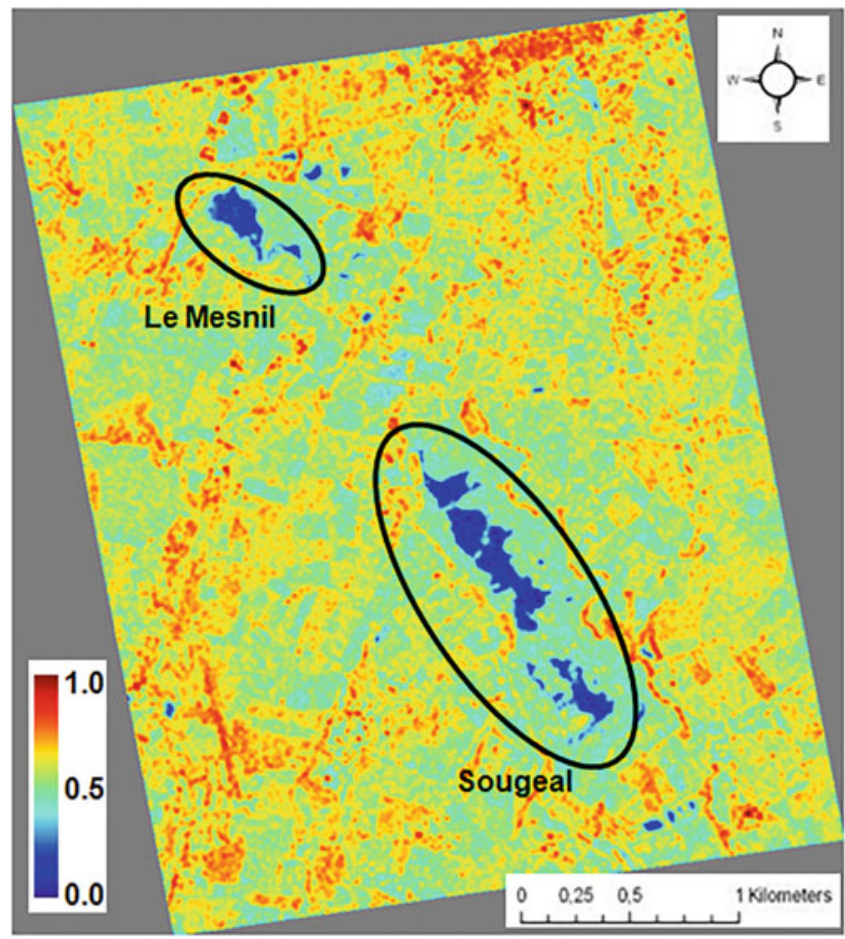

Fig. 3.26 The normalised Shannon entropy (SE) image in the case of the target vector $\left[S_{h v}, S_{v v}\right]^{T}$

dynamics of wetlands can be seriously considered as a concrete application for the RADARSAT-RCM and Sentinel-1 missions.

\subsubsection{Discussion on the Role of Polarisation, on the Maturity of the Application and Conclusions}

The aim of this showcase is to determine the capabilities of fully polarimetric SAR time series data sets to delineate and monitor wetland ecosystems. This study was set from February 2010 to February 2011 (15 images) over a wetland area located in France (Brittany). Every 24 days, simultaneously with the image acquisition in a Fine Quad-Pol mode (beam FQ23, 42.6 ${ }^{\circ}$ ), a 1-year ground truth measurement campaign to measure the distance between a reference fix point and the limit of the water table has been conducted. It has been established in this study that the Shannon entropy is a parameter very sensitive to the temporal variability of flooded areas. Maps of the fluctuated trend of the open water from a 1-year RADARSAT-2 quad-pol time series have been achieved, in an automatic procedure, from the segmentation of this polarimetric descriptor. A lidar image of the investigated area, providing very accurate elevation and precise microtopography information, has corroborated these results, leading to a very fine analysis of the obtained results. The limits of the water bodies extracted from segmented images highlight inundation extend oscillation, which is a key environmental factor controlling ecological, hydrological and chemical processes. This product, when generated over several years, can provide useful information to characterise the water cycle and identify variations that may affect the overall ecosystem. The results presented here show already the important potential of fully polarimetric SAR data. However, a very promising result concerns the very good correlation between the dual-polarimetric Shannon entropy and the fully polarimetric one, which means that it will also be possible to extract the open water and its spatial distribution when this proposed methodology will be applied on the future dual polarimetry RADARSAT-RCM and Sentinel-1 time series data sets. At last, it is important to note that this proposed methodology cannot be applied to single-polarisation data. Indeed, single-pol data processing is often and/or only based on the analysis of the backscattering level, and in such a case, single-pol data become very ambiguous for the delineation of wetlands since the same backscatter level can be reached at land and water, depending on the wind conditions. Water bodies become a rougher surface and so return a stronger signal in presence of wind.

\subsubsection{Tropical Wetland Characterisation with Polarimetric SAR}

\subsubsection{Introduction, Motivation and Literature Review}

Tropical wetlands are very productive ecosystems located mainly in the tropics, but can also be found in sub-tropical regions, where temperatures sometimes drop below freezing 
point. They consist of both inland freshwater ecosystems and coastal, mangrove systems. Both ecosystems serve as a critical habitat for a wide variety of plant and animal species. Tropical wetlands also have a valuable economical importance, as they serve as a large carbon reservoir, filter nutrients and pollutants and provide aquatic habitats for outdoor recreation, tourism and fishing (Barbier 1994). Globally, many tropical wetlands are under severe environmental stress, mainly from agricultural and urban development, pollution and rising sea level.

In order to generate an inventory of tropical wetlands and assess their health, it is important to map, characterise and monitor these fragile ecosystems. Such tasks are best conducted using remote sensing observations, because of the remoteness and large extent of many tropical wetlands. Optical remote sensing observations are fairly limited in the tropics, because of frequent cloud coverage. The all-weather capability synthetic aperture radar (SAR) observations are very useful for the characterisation and monitoring tasks, especially quadruple-polarimetric (quad-pol) data that contains more spatial information related to physical characteristics than single- or dual-polarimetric data.

A common method for characterising landscapes with polarimetric SAR (PolSAR) observations is decomposition analysis, which maps the landscape according to the surface's scattering mechanisms. In this contribution we use four decomposition types, Pauli, Freeman, Yamaguchi and Hong and Wdowinski. The first three decompositions, Pauli, Freeman and Yamaguchi, have been applied successfully to characterise landscapes in various environments. The fourth decomposition by Hong and Wdowinski (H\&W) is a new decomposition, which was developed in accordance with new SAR phase observations (interferograms) in tropical wetlands. The contribution includes a brief description of the new decomposition and the application of all four decompositions to the following four quad-pol data sets acquired over the Everglades: three satellites, TerraSAR-X, RADARSAT-2 and ALOS-PALSAR, and one airborne sensor UAVSAR. The results of our decomposition analyses indicate that quad-pol SAR data is very useful for tropical wetland characterisation, in particular the X- and L-band data sets.

Quad-pol SAR data have been used over the past two decades to study and characterise tropical wetlands in various locations worldwide. The early quad-pol PolSAR studies relied on airborne $\mathrm{C}$-, L- and P-band observations acquired by NASA's AIRSAR mission over tropical wetlands in Belize, French Guiana, Australia and the USA. Most of these studies focused on the interaction of the SAR signal with mangrove vegetation and the possibility to retrieve quantitative estimates of biophysical parameters such as tree height and biomass (Pope et al. 1994; Proisy et al. 2000; Lucas et al. 2007; Trisasongko 2009). The AIRSAR data was also used to map sub-environments of coastal herbaceous wetlands (Slatton et al. 1996). Another more advanced airborne sensor that acquired quad-pol data over tropical wetlands is NASA's UAVSAR, which operates at L-band. Fatoyinbo and Simard (2011) used quad-pol UAVSAR combined with ALOS polarimetric data to estimate biomass of mangrove forests in various remote locations in the tropics.

The use of space-borne quad-pol data for tropical wetland studies has been, so far, fairly limited due to the limited amount of space-borne quad-pol data acquired over tropical wetlands. The shuttle imaging radar-C (SIR-C) was the first space-borne sensor to acquire quad-pol SAR data. The SIR-C data were used to detect a seasonal flooding component in Yucatan wetlands (Pope et al. 1997) and for land cover classification in India (Turkar and Rao 2011). Insofar only three satellites acquired quad-pol SAR data, two of them in an experimental mode (TerraSAR-X and ALOS-PALSAR) and one, RADARSAT-2, as part of its regular operation. Ullmann et al. (2012) used quad-pol TerraSAR-X data acquired over Indonesia and analysed them using Cloude and Yamaguchi decompositions. Their analyses revealed land classification in which wetlands and other flooded vegetation could easily be identified by the double-bounce scattering component. Hong et al. (2010) and Hong and Wdowinski (2011) used RADARSAT-2 quad-pol data acquired over the Everglades wetlands in south Florida. Interferometric processing of the data revealed a very similar fringe pattern in all polarisation interferograms suggesting that both co- and cross-pol signals sample the water surface beneath the vegetation. Based on the phase observations, Hong and Wdowinski (2013) developed a new PolSAR decomposition analysis with a double-bounce component from cross-polar channel.

Decomposition analyses are common methods for characterising landscape with polarimetric SAR observations. The widely used Pauli decomposition is a simple method that represents the main three scattering mechanisms: singlebounce, double-bounce and volume scattering (Cloude 2009; Cloude and Pottier 1996; Lee and Pottier 2009). A threecomponent scattering model proposed by Freeman and Durden (1998) has been successfully applied to decompose polarised SAR data into three components according to the main scattering mechanisms under reflection symmetry conditions. In order to account for non-reflection symmetry conditions, Yamaguchi et al. $(2005,2006)$ added a fourth helix component to their decomposition. Various methods were proposed to estimate the volume scattering component considering non-reflection symmetry condition (Arii et al. 2010, 2011; Neumann et al. 2009). Recently an extended volume scattering model was discussed using randomly orientated diplane scatterers (Sato et al. 2012a). More recent decomposition studies also included mathematical operations on the decomposed coherency matrix in order to resolve anomalous 
Table 3.7 List of decompositions used in this study and their main characteristics

\begin{tabular}{l|l|l}
\hline Decomposition & Characteristics & References \\
\hline Pauli & 3 components: HH-VV, HH + VV, HV & Cloude and Pottier (1996) \\
\hline Freeman & Model based, 3 components: single bounce, double bounce, volume & Freeman and Durden (1998) \\
\hline Yamaguchi & Model based, 4 components: single bounce, double bounce, volume, helix & Yamaguchi et al. (2005) \\
\hline H\&W & Model based, 3 components: single bounce, double bounce (co- and cross-pol), volume & Hong and Wdowinski (2013) \\
\hline
\end{tabular}

values generated by the previous three- and fourdecomposition methods (Yamaguchi et al. 2011; van Zyl et al. 2011; Lee and Ainsworth 2011). Touzi et al. (2009) proposed a phase of target scattering decomposition for wetland characterisation, and Hong and Wdowinski (2013) developed a new decomposition analysis with a double-bounce component from cross-pol.

\subsubsection{Methodology}

Our methodology for characterising tropical wetlands with quad-pol SAR data consists of four decomposition analyses, Pauli, Freeman, Yamaguchi and H\&W (Table 3.7). The first decomposition we considered, Pauli, is a simple method that represents the main three scattering mechanisms. The other three decompositions are model-based, accounting for three or four scattering mechanisms. The Freeman decomposition accounts for three mechanisms (single bounce, double bounce and volume), whereas the Yamaguchi decomposition includes a fourth helix scattering component. The H\&W decomposes the cross-pol signal into volume and doublebounce scattering components, which increases the doublebounce scattering component on the account of volume scattering. The inclusion of a cross-pol double-bounce scattering is based on cross-pol SAR phase observations in wetlands showing coherent interferometric signal backscattered from the water surface beneath the vegetation. Touzi et al. (2009) presented a non-model, roll-invariant target scattering decomposition for the characterisation of temperate wetlands. We did not include Touzi decomposition in this study, because we focus on model-based decompositions.

The full description and mathematical formulation of the four decompositions can be found in the references listed in Table 3.7. The first three listed decompositions are wellknown and do not need much introduction. However, the fourth decomposition, $\mathrm{H} \& \mathrm{~W}$, is fairly new. Hence we provide a brief description of the decomposition, including key equations. Our decomposition is based on phase observations from wetlands indicating that the cross-pol signal samples the water surface beneath the vegetation. The simplest scattering mechanism that accounts for scattering in the cross-pol signal is a rotated dihedral, which we included in our formulation. Following the formulation of Yamaguchi et al. (2005), we utilise a $3 \times 3$ coherency matrix to derive each scattering component mathematically as shown in Eq. (3.8):

$$
\begin{aligned}
& \langle\mathbf{T}\rangle^{H V}=\left[\begin{array}{ccc}
T_{11} & T_{12} & T_{13} \\
T_{21} & T_{22} & T_{23} \\
T_{31} & T_{32} & T_{33}
\end{array}\right]= \\
& =f_{s} \mathbf{T}_{\text {single }}^{h v}+f_{d} \mathbf{T}_{\text {double }}^{h v}+f_{v} \mathbf{T}_{\text {volume }}^{h v}+f_{d} \mathbf{T}_{\text {rotated_diplane }}^{h v}
\end{aligned}
$$

where \langle\rangle denotes the ensemble average of a few pixels in the data processing and $f_{s}, f_{d}$ and $f_{v}$ are the coefficients related to the powers of single-bounce, double-bounce and volume scattering components, respectively.

Following Yamaguchi et al. (2005) four-component decomposition formulation, we adopted the same scattering coherency matrices for the single-bounce, double-bounce and volume scattering as follows:

$$
\begin{aligned}
\langle\mathbf{T}\rangle_{\text {single }}^{h v}= & {\left[\begin{array}{ccc}
1 & \beta^{*} & 0 \\
\beta & |\beta|^{2} & 0 \\
0 & 0 & 0
\end{array}\right] } \\
\langle\mathbf{T}\rangle_{\text {double }}^{h v} & =\left[\begin{array}{ccc}
|\alpha|^{2} & \alpha & 0 \\
\alpha^{*} & 1 & 0 \\
0 & 0 & 0
\end{array}\right] \\
\langle\mathbf{T}\rangle_{\text {volume }}^{h v} & =\frac{1}{4}\left[\begin{array}{lll}
2 & 0 & 0 \\
0 & 1 & 0 \\
0 & 0 & 1
\end{array}\right]
\end{aligned}
$$

We introduce a double-bounce scattering model of a rotated dihedral for the cross-pol signal, with the following matrix which is integrated with the probability density function similar to formulations of Yamaguchi et al. (2005):

$$
\begin{aligned}
\mathbf{S}^{H V} & =\left[\begin{array}{cc}
1 & 0 \\
0 & -1
\end{array}\right] \text { or } \mathbf{S}^{H V}=\left[\begin{array}{cc}
-1 & 0 \\
0 & 1
\end{array}\right] \\
& \Rightarrow\langle\mathbf{T}\rangle_{\text {rotated_diplane }}^{h v}=\frac{1}{2}\left[\begin{array}{lll}
0 & 0 & 0 \\
0 & 1 & 0 \\
0 & 0 & 1
\end{array}\right]
\end{aligned}
$$

Equation (3.10) allows us to estimate the doublebounce component in the cross-pol signal. Thus, the 
coherency matrix in Eq. (3.8) can now be written as a combination of single-bounce, double-bounce from both co- and cross-pol and volume scattering components as follows:

$$
\begin{aligned}
& \langle\mathbf{T}\rangle^{H V}=f_{s} \mathbf{T}_{\text {single }}^{h v}+f_{d} \mathbf{T}_{\text {double }}^{h v}+f_{v} \mathbf{T}_{\text {volume }}^{h v}+f_{d} \mathbf{T}_{\text {rotated_diplane }}^{h v}= \\
& =f_{s}\left[\begin{array}{ccc}
1 & \beta^{*} & 0 \\
\beta & |\beta|^{2} & 0 \\
0 & 0 & 0
\end{array}\right]+f_{d}\left[\begin{array}{ccc}
|\alpha|^{2} & \alpha & 0 \\
\alpha^{*} & 1 & 0 \\
0 & 0 & 0
\end{array}\right]+\frac{f_{v}}{4}\left[\begin{array}{lll}
2 & 0 & 0 \\
0 & 1 & 0 \\
0 & 0 & 1
\end{array}\right]+\frac{f_{d}}{2}\left[\begin{array}{ccc}
0 & 0 & 0 \\
0 & 1 & 0 \\
0 & 0 & 1
\end{array}\right]
\end{aligned}
$$

We have four observables and five unknown parameters in Eq. (3.11). We reduce the number of unknown parameters by assuming $\alpha=0$ in the areas dominated by surface scattering and $\beta^{*}=0$ in areas dominated by double-bounce scattering. Further details of the decomposition are provided by (Hong and Wdowinski 2013).

\subsubsection{Experimental Results}

Test sites and corresponding radar and validation data sets selected for tropical wetland characterisation and monitoring are summarised in Table 3.8 and further described in the Appendix.

We applied the 4 decompositions listed in Table 3.7 to the 4 quad-pol data sets listed in Table 3.8 and obtained 16 different decompositions that characterise our study area in the Everglades. In order to evaluate the decomposition results, we first present the four decompositions applied to the RADARSAT-2 data set and compare the results with Landsat image and detailed vegetation map (Fig. 3.27). We then compare three decomposition results applied to three data sets that were acquired with three different SAR frequencies (X-, C- and L-bands), covering the same area (Fig. 3.27). In this comparison we applied the $\mathrm{H} \& \mathrm{~W}$ decomposition to all data sets. Again, we compare the decomposition results with an optical Landsat image and a vegetation map.

The study of the RADARSAT-2 data set (Fig. 3.27) shows that all four decompositions yield similar results indicating double-bounce scattering (red) in the northeast corner of the data set, combined volume and single-bounce scattering (blue-green) in the southwest corner and mostly volume scattering (green) in a wide area located between the two corners. There are some variations in the decompositions. The most noticeable difference is the high volume scattering of the Freeman decomposition (Fig. 3.27b). The issue of high volume scattering (too much green) in urban environments led (Yamaguchi et al. 2005) to develop their four-component decomposition with helix scattering, which indeed shows less volume scattering. The H\&W decomposition also shows less volume scattering than the Freeman one, because some of the cross-pol signal contributes to the double-bounce component. We validate the decomposition results by comparing them to an optical Landsat image (Fig. 3.27e) and vegetation map of the study area (Fig. 3.27f). A visual comparison between the decompositions and vegetation map indicates that all four decompositions characterised well the mangrove forests in the southwest corner and the sawgrass in the northeast corner, as both areas contain fairly homogeneous vegetation. The decompositions' spotty patterns in the transition zone between the saltwater mangroves and freshwater sawgrass reflect the mixture of vegetation types in that transition zone. Both prairies and scrub vegetation types consist of a variety of grass, bushes and trees, which results in a spotty pattern in the decomposition.

The multi-frequency decomposition study (Fig. 3.28) yields very interesting results, as in each frequency the radar signal interacts differently with the vegetation. In this study we show results of the Hong and Wdowinski decomposition; similar results were obtained by the other

Table 3.8 Test sites and corresponding radar and validation data selected for the generation of showcases on tropical wetland characterisation

\begin{tabular}{l|l|l}
\hline Application/product & Test site - radar data & Reference data \\
\hline \multirow{2}{*}{ Tropical wetland characterisation } & Shark River Slough & Optical data, vegetation maps \\
\cline { 2 - 2 } & TerraSAR-X, 16/04/2010 & \\
\cline { 2 - 3 } & RADARSAT-2, 23/09/2008 & \\
\cline { 2 - 3 } & ALOS-PALSAR, 19/04/2011 & \\
\cline { 2 - 3 } & Coastal mangrove forests & \\
\cline { 2 - 3 } & UAVSAR, L-band, 22/06/2010 & \\
\hline
\end{tabular}




\section{(a) Pauli}

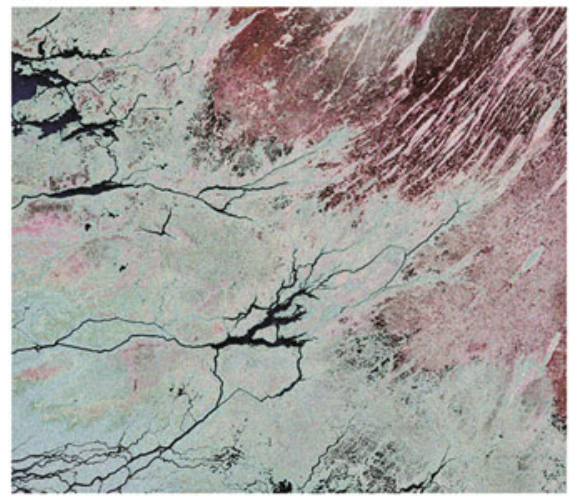

(d) H\&W

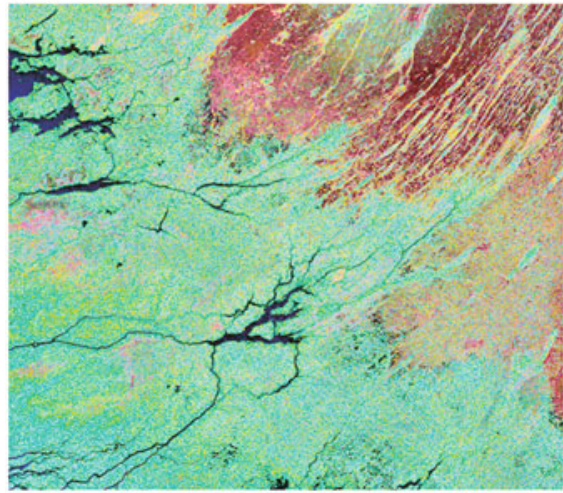

\section{(b) Freeman}

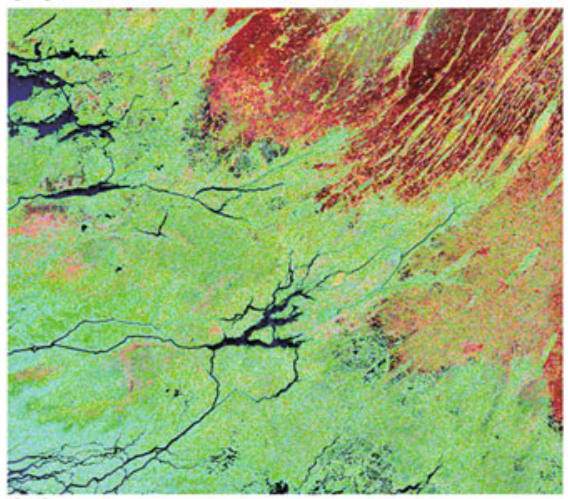

(e) Landsat

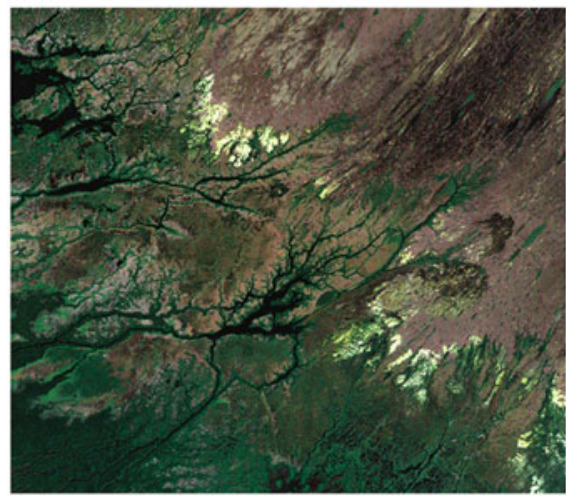

(c) Yamaguchi

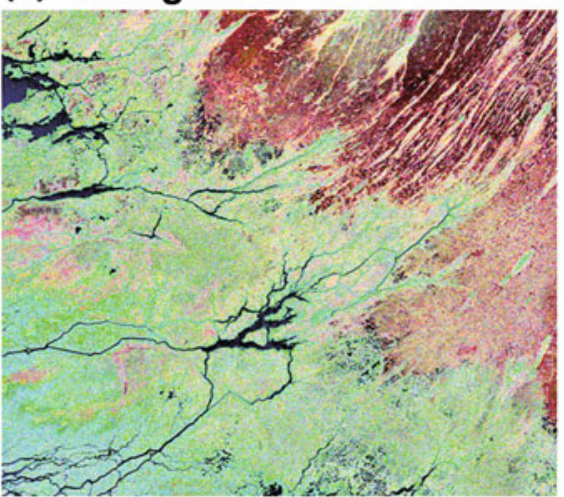

(f) Vegetation map

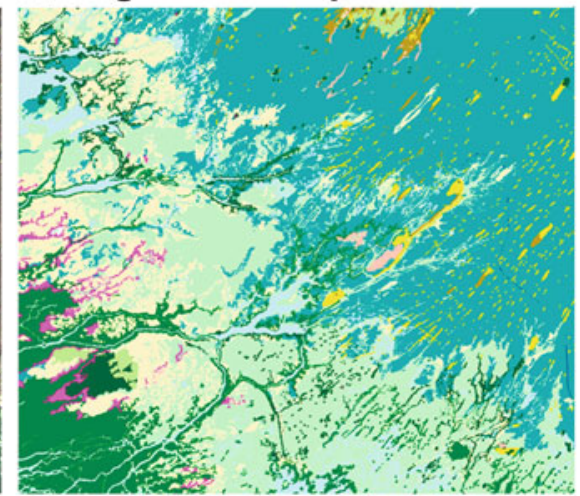

Fig. 3.27 Polarimetric decomposition results of the C-band RADARSAT-2 data, acquired on 23rd of September 2008. (a) Pauli decomposition presented as colour composite image with the following decomposition components: $\mathrm{HH}-\mathrm{VV}$ (red), $\mathrm{HH}+\mathrm{VV}$ (blue) and $\mathrm{HV}$ (green). (b) Freeman's three-component decomposition with the following components: blue, single bounce; red, double bounce; and green,

decompositions. The decomposition of the TSX data set shows large colour variability reflecting a high sensitivity level of the X-band $(3.1 \mathrm{~cm})$ to distinguish between the various vegetation types. The decomposition shows in great details the distribution of mangroves along the tidal channels as volume scattering, the sawgrass in the northeast corner as double-bounce scattering and a mixture of all three scattering mechanisms in this vegetation transition zone between freshwater (sawgrass) and saltwater (mangroves) ecosystems. The decomposition of the RADARSAT-2 data set shows a dominance of volume scattering (green) throughout the image and, hence, low resolution to distinguish between the various vegetation types in the transition zone. Nevertheless, the decomposition still distinguishes well the sawgrass (red in the northeast corner) from the rest of the vegetation types. The L-band decomposition also shows high variability and sensitivity to different vegetation types.

The most interesting difference between the ALOS and the other two decompositions is the sawgrass characterisation in northeast corner. The ALOS decomposition characterises the sawgrass area as single-bounce scattering (blue), whereas volume scattering. (c) Yamaguchi's four-component decomposition with same colour scheme as in (b). (d) Hong and Wdowinski's threecomponent decomposition with cross-pol double bounce. (e) Landsat-7 $\mathrm{ETM}+$ optic colour composite image. (f) Vegetation map of the study area (Florida Coastal Everglades, Long Term Ecological Research (FCE LTER, http://fcelter.fiu.edu)). Legend is shown in Fig. 3.28 the other two decompositions as double bounce. The different scattering mechanism reflects a different interaction of the relatively thin sawgrass (a few $\mathrm{cm}$ wide) with the different wavelengths of the radar signal. The sawgrass serves as good scatterers for the X- and C-band signals $(3.1$ and $5.6 \mathrm{~cm}$, respectively), but is transparent to the longer wavelength L-band signal $(24.1 \mathrm{~cm})$.

The validation of the three decompositions with the Landsat image and vegetation maps indicates a very good sensitivity of the TSX and ALOS decompositions to characterise this vegetation transition zone. Furthermore, the two decompositions show higher variability than the vegetation map, which can be used for a better vegetation classification of tropical wetlands. The lower performance of the RADARSAT-2 decomposition is surprising, because the C-band data corresponds to an intermediate frequency/wavelength between the X-and L-band data, which both revealed high sensitivity to tropical wetland vegetation. In order to verify these results, we checked five different RADARSAT2 quad-pol data sets acquired over the same area at different times with all four decompositions. The results of all 


\section{(a) TerraSAR-X}

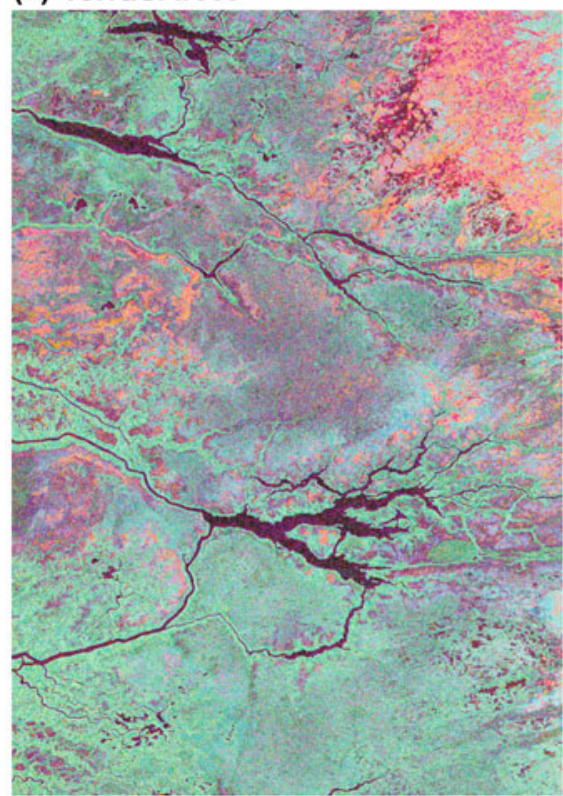

(d) Landsat

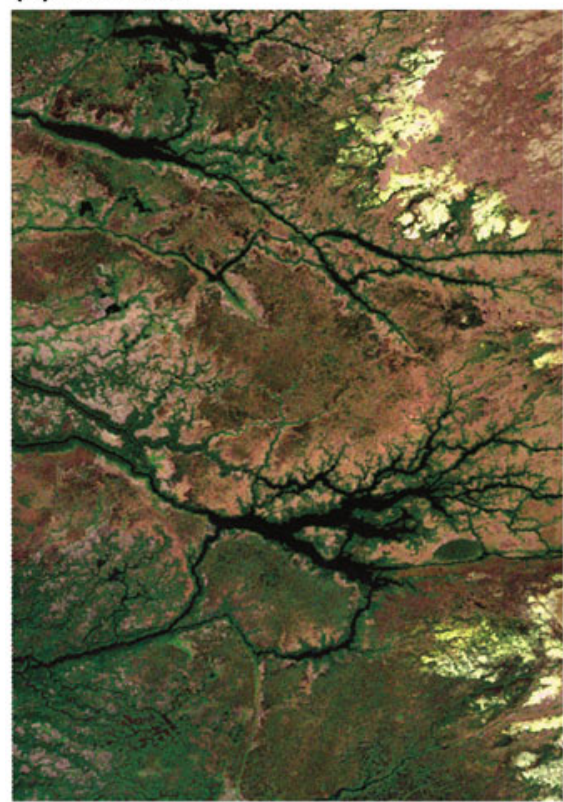

(b) Radarsat-2

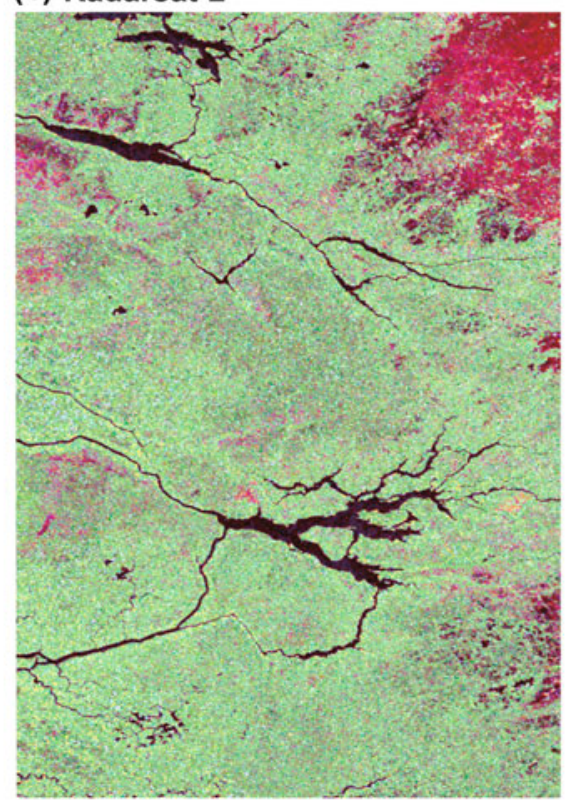

(e) Vegetation map

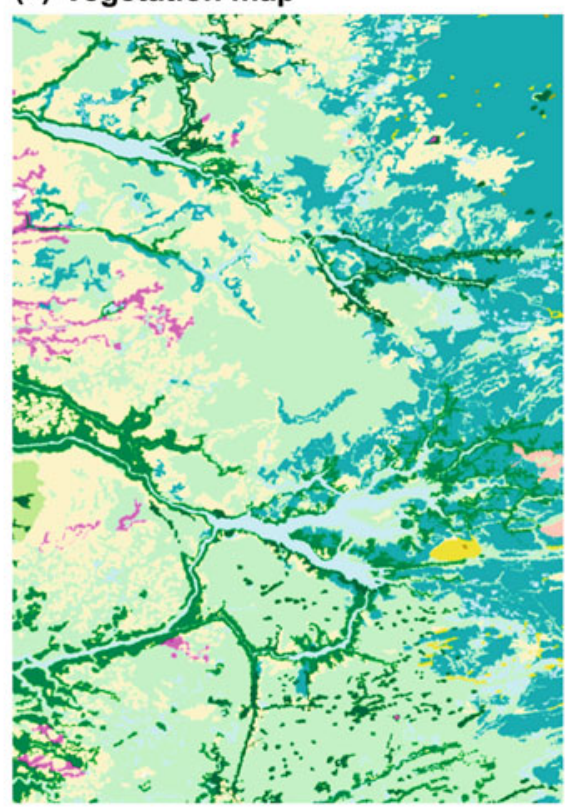

(c) ALOS

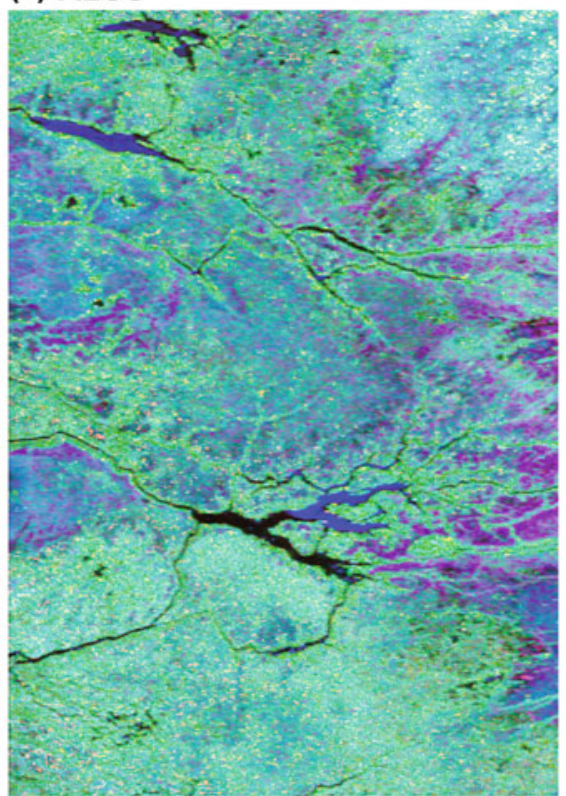

Fig. 3.28 Decomposition colour composite image of the Shark River Slough site in the Everglades using Hong-Wdowinski's approach (blue, single bounce; red, double bounce (both from the co- and the cross-pol); and green, volume scattering) of the quad-pol data sets: (a) X-band
TerraSAR-X, (b) C-band RADARSAT-2 and (c) L-band ALOSPALSAR. (d) Landsat-7 ETM+ optic colour composite image and (e) vegetation maps (Florida Coastal Everglades, Long Term Ecological Research (FCE LTER), http://fcelter.fiu.edu) decompositions are very similar to those presented here in Fig. 3.28b. A possible explanation for the lower performance of the C-band data is the vegetation wavelength that promotes Bragg scattering in C-band, as suggested by Atwood et al. (2013).

The analysis of the UAVSAR data set with four decompositions is presented in Fig. 3.29. The data covers a long swath of mainly mangrove forest located along the western coast of south Florida. The decompositions yielded similar results to that presented in Fig. 3.27, in which the Yamaguchi and $\mathrm{H} \& \mathrm{~W}$ decompositions show a better fit to the vegetation map.

\subsubsection{Comparison with Single-/ Dual-Polarisation Data}

The use of single- or dual-polarimetric data to characterise tropical wetlands yielded variable quality results, depending the radar frequency, image resolution, hydrological 
(a) Pauli

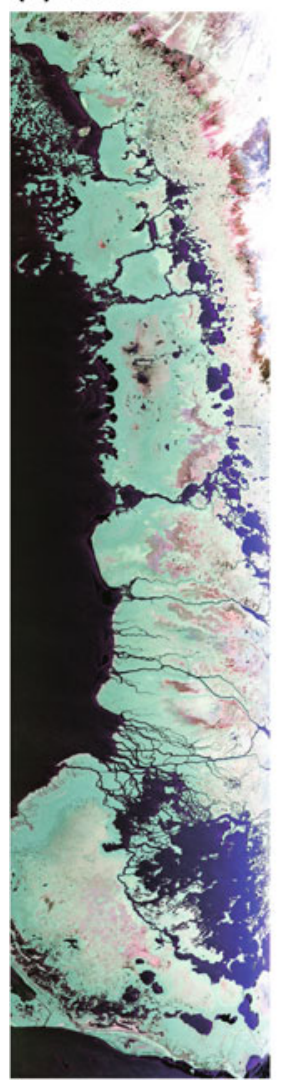

(b) Freeman

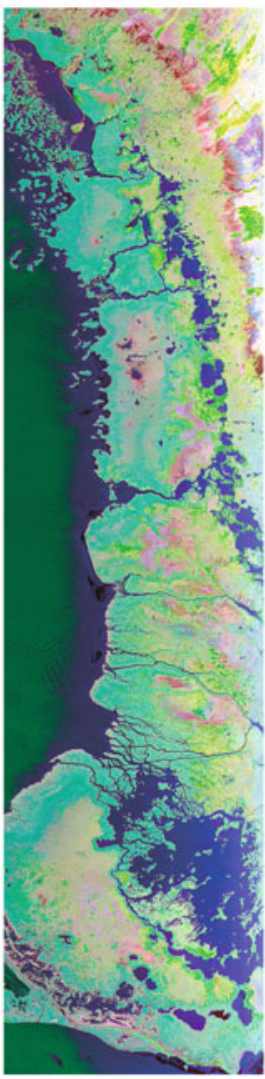

(c) Yamaguchi

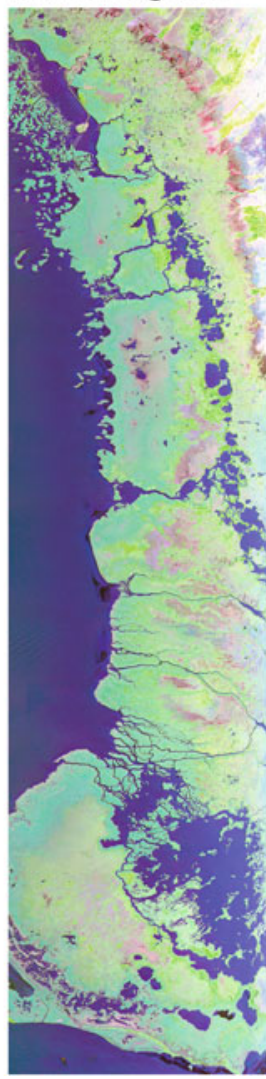

(d) H\&W

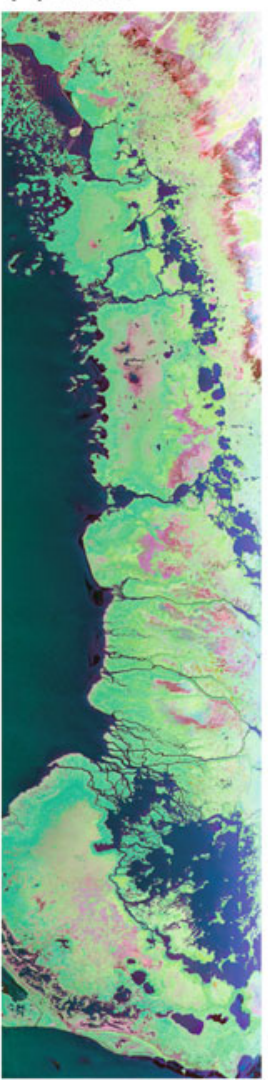

(e) Landsat

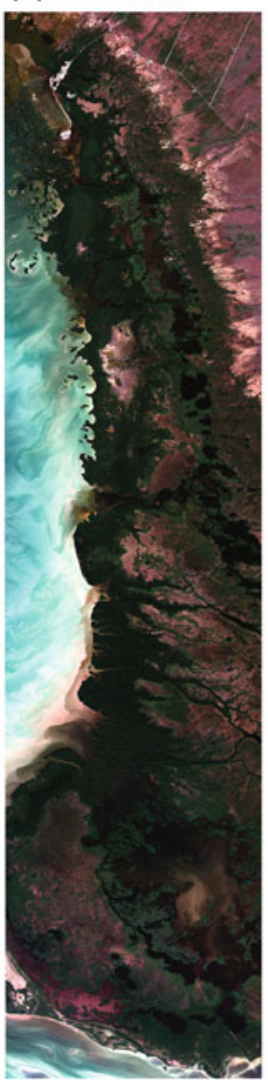

(f) Vegetation map

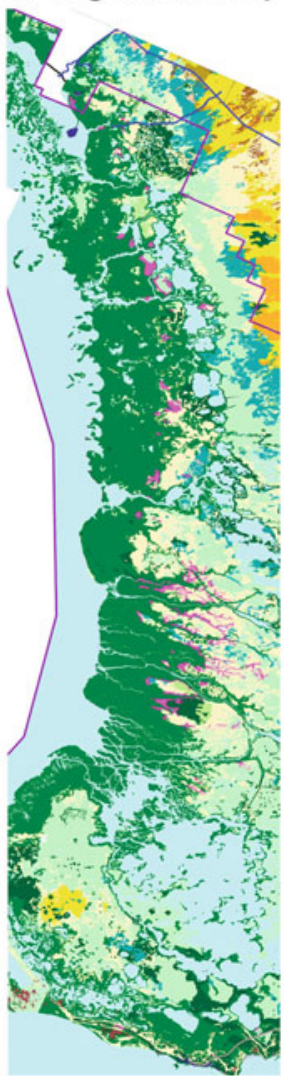

Fig. 3.29 Polarimetric decomposition results of the L-band UAVSAR data, acquired on 19th of April 2011. (a) Pauli decomposition presented as colour composite image with the following decomposition components: $\mathrm{HH}-\mathrm{VV}$ (red), $\mathrm{HH}+\mathrm{VV}$ (blue), and $\mathrm{HV}$ (green). (b) Freeman's three-component decomposition with the following components: blue, single bounce; red, double bounce; and green,

conditions and vegetation type (e.g. Bourgeau-Chavez et al. 2005). Single- and dual-pol can distinguish fairly well between woody and herbaceous vegetation and was successfully used to map mangrove forest distribution (e.g. Lucas et al. 2007). However, single- and dual-pol data are often not sufficient to distinguish between non-homogeneous vegetation distributions. Thus recent studies suggest the use of single- and dual-pol data combined with optical data for wetland characterisation (Bourgeau-Chavez et al. 2009; Evans and Costa 2013). Because quad-pol data contains twice or quadruple amount of data than dual- or single-pol data, the usage of quad-pol data might be sufficient for characterising tropical wetlands.

The examples presented in this study support the idea that quad-pol data can be sufficient for precise wetland characterisation, as the examples indicate high sensitivity of the various decomposition results to the different vegetation types. To illustrate the strength of the quad-pol characterisation, we compared our decomposition results with single- and dual-pol representations of the same wetland

volume scattering. (c) Yamaguchi's four-component decomposition with same colour scheme as in (b). (d) Hong and Wdowinski's threecomponent decomposition with cross-pol double bounce. (e) Landsat-7 ETM+ optic colour composite image. (f) Vegetation map of the study area. Legend is shown in Fig. 3.29

area (Fig. 3.30). The comparison shows that the single- and dual-pol images have limited ability to distinguish between the different vegetation types. The grey scale of single-pol image allows us to distinguish between 3 and 4 shades of grey corresponding to different vegetation types. The dualpol image that combines two ranges of colours (red and green) can distinguish 6-7 areas of different scattering characteristics. The quad-pol decomposition uses three ranges of colours (red, blue and green), which provide sufficient sensitivity to distinguish ten or more areas of different scattering characteristics, which are sufficient to characterise the main vegetation types in tropical wetlands.

\subsubsection{Discussion on the Role of Polarimetry, on the Maturity of the Application and Conclusions}

Decomposition analyses have been used for more than a decade to characterise landscapes according to scattering mechanisms. In that sense, it is a mature application for most landscape characterisation. However, the use of 


\section{(a) Single-pol: $\mathrm{HH}$}

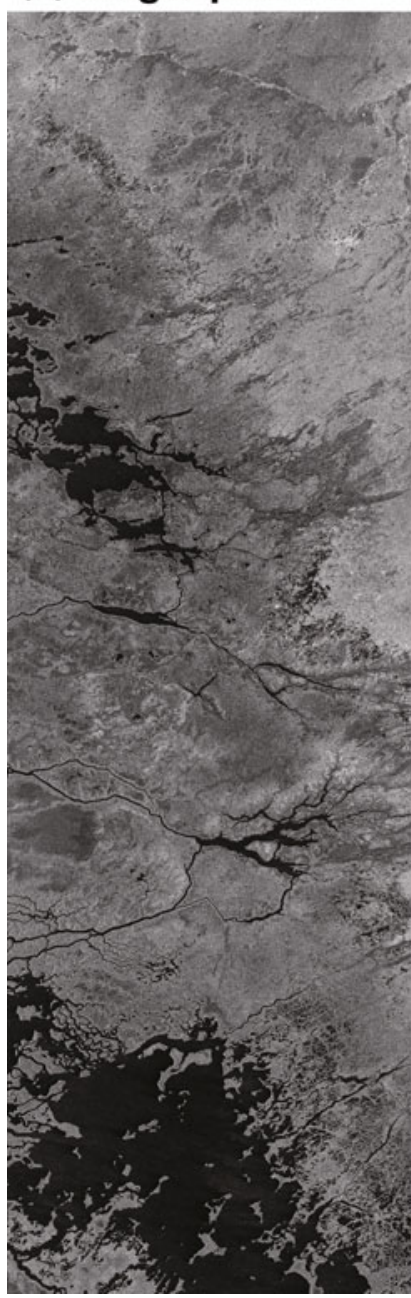

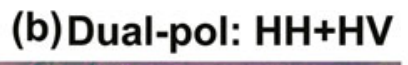

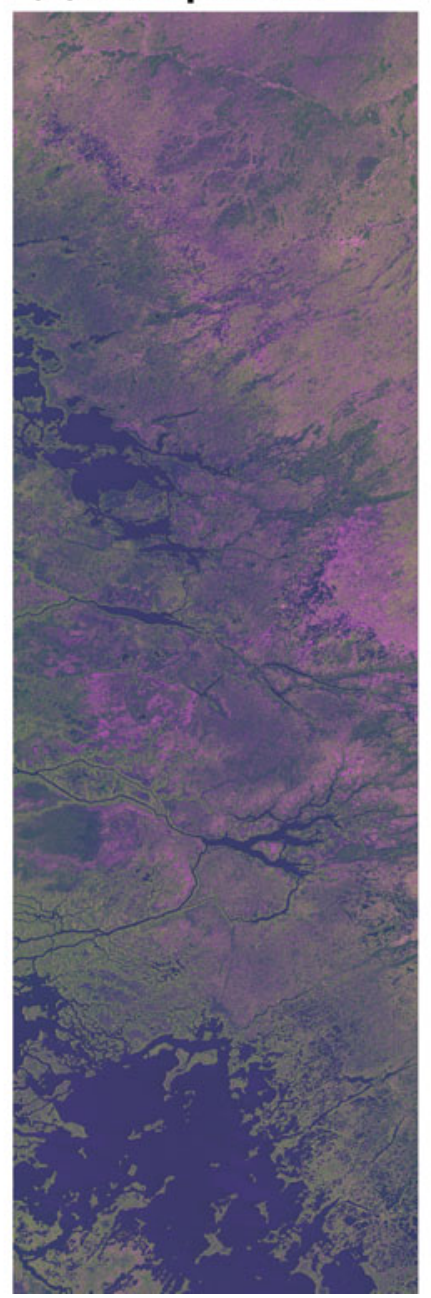

(c) Quad-pol: Pauli

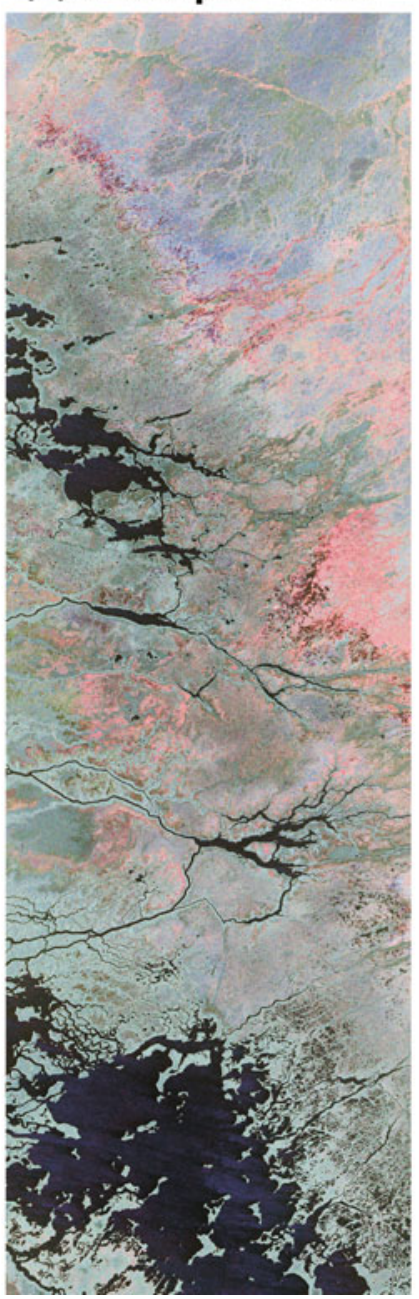

(d)Quad-pol: H\&W

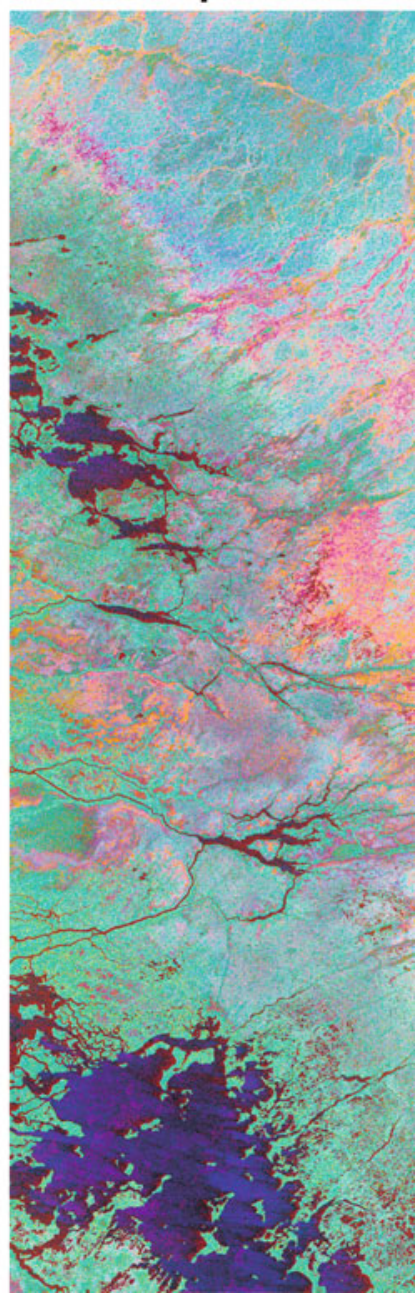

Fig. 3.30 Comparison between single-, dual- and quad-pol characterisation of tropical wetland area. (a) Amplitude image of single-polarimetric data of TerraSAR-X. (b) Colour composite image of dual-polarimetric data: HH (red), HV (green) and HH-HV (blue). (c) decompositions for characterising tropical wetlands was rather limited due to the limited available quad-pol data acquired over these wetlands. In this study we demonstrated that quad-pol decompositions are very useful methods for the characterisation of tropical wetlands. We analysed four quadpol data sets using four decomposition types. All decompositions were able to distinguish well between the main vegetation types, which include saltwater mangrove forests, freshwater sawgrass swamp and a transition zone with a mixture of vegetation types. Our analyses indicate that the TSX and ALOS quad-pol decompositions are more sensitive to vegetation variation in the transition zone than the RADARSAT-2 decomposition. Both TSX and ALOS decompositions show more details of vegetation distribution that the available vegetation cover map.
Pauli decomposition colour composite image: $\mathrm{HH}-\mathrm{VV}$ (red), $\mathrm{HH}+\mathrm{VV}$ (blue) and HV (green). (d) Hong and Wdowinski's three-component decomposition: double bounce (red), surface bounce (blue) and volume scattering (green)

\subsubsection{Subarctic Peatland Characterisation and Monitoring}

\subsubsection{Introduction, Motivation and Literature Review}

Wetlands play a key role in regional and global environments and are critically linked to major issues such as climate change, water quality, the hydrological and carbon cycles and wildlife habitat and biodiversity. Wetlands with at least 30-40 centimetres of peat accumulated on the surface represent an important class of wetland named peatland. Although peatlands globally only cover $3 \%$ of the land, they store $30 \%$ of the terrestrial carbon (Gorham 1995). Therefore, it is important to maintain and protect peatlands to reduce greenhouse gases. 
Unfortunately, major peatland transformations have been detected in the boreal and subarctic peatland regions. While it is well established that fens change naturally into bogs over time and that bogs can revert to fens, the observations over the last 50 years indicate that the rate of these changes has been significantly altered by various sources of stress (climate change, isostatic uplift, fire and anthropogenic activities) and this could have important implications (Gorham 1995; Brook and Kenkel 2002; Brook 2006; Jano et al. 1998). This has been noted in the Hudson Bay Lowlands of Manitoba, Canada, which contain the most extensive wetlands and thickest peat deposits in Canada (Brook and Kenkel 2002; Brook 2006; Jano et al. 1998). This region is home to unique concentrations of wildlife, most notably polar bears and caribous. Bears rely on inland denning habitat in the peatlands, and caribous are tied to peatland vegetation (bogs in winter and fens in summer). The loss of bogs will have important implications for polar bear denning habitat which is entirely within bogs with thick peat deposits (Brook and Kenkel 2002; Brook 2006; Jano et al. 1998). This is in addition to the impact on global warming that would occur due to the release of the carbon stored by peatlands. Therefore, there is an immediate need for cost-effective tools that permit accurate classification and mapping of peatlands, as well as long-term monitoring of their (bog-fen) transformations.

Cost-effective wetland characterisation and monitoring are now possible due to advances in the technology of earth observation satellites that provide the possibility of efficient monitoring of wetland status over large and remote areas (Fournier et al. 2007; Grenier et al. 2007). Recently, the visible near-infrared (VNIR) satellites (and Landsat in particular) have become the most popular source of information for wetland mapping. In Canada, Landsat 7 data combined with RADARSAT1 HH-polarisation SAR data have been used as the basis source of information for building the Canadian Wetland Inventory (Fournier et al. 2007; Grenier et al. 2007). The use of the additional all-weather single polarisation $(\mathrm{HH})$ RADARSAT1 information permits better delineation and monitoring of wetland water extent (Touzi et al. 2009; Grenier et al. 2007). Unfortunately, the combination with VNIR optical and SAR satellite information cannot clearly discriminate bogs from poor (shrubs and sedges) fens (Fournier et al. 2007; Grenier et al. 2007; Touzi et al. 2007). As a result, bogs are generally confused with fens in the North, and the bog to fen transformations related to climate change or anthropogenic activities may not be detected.

Since bog and fen vegetation can hardly be discriminated by optic and conventional SAR sensors, it might be interesting to use their different hydrological properties to discriminate these two peatland classes. In fact, fens and bogs differ in the way that they receive water and therefore receive nutrients differently. A bog is a peat-forming ecosystem that lacks any significant groundwater inflow and is therefore ombrotrophic. The bog soils are not affected by the mineralenriched ground waters from surrounding soils, since precipitation, fog and snow are the primary water sources. In contrast to the ombrotrophic bogs, the minerotrophic fens are connected to small streams and may also receive water from surrounding uplands (Ingram 1982; Zoltai and Vitt 1995). In this study, we will show that earth observation satellites equipped with L-band polarimetric SAR could provide the required information for cost-effective peatland mapping and monitoring in the boreal and subarctic peatlands. The unique capability of polarimetric SAR for the detection of peatland subsurface water flow, first demonstrated in (Touzi et al. 2007), could permit an enhanced discrimination of bog from fen, which will result in better monitoring of bog-fen transformations related to the various (natural and anthropogenic) sources of stress. This will be confirmed herein using polarimetric L-band ALOS collected over a boreal peatland, La Baie des Mines, and subarctic peatlands in the Wapusk National Park in Canada.

Several studies have been published on the investigation of polarimetric SAR for wetland classification (Pope et al. 1994, 1997; Hess et al. 1995; Sokol et al. 2004). Pope et al. $(1994,1997)$ have shown that the phase difference between the $\mathrm{HH}$ and $\mathrm{VV}$ polarisations, $\phi_{\mathrm{HH}}-\phi_{\mathrm{VV}}$, was the most useful parameter for flooded wetland classification and detection of seasonal flooding wetland changes. $\phi_{\mathrm{HH}}-\phi_{\mathrm{VV}}$, which characterises wetland scattering in terms of odd and even bounce interactions (Van Zyl 1989), permits an enhanced identification of marsh classes generally dominated by double-bounce interactions. However, they raised the point that the high variability of $\phi_{\mathrm{HH}}-\phi_{\mathrm{VV}}$ might limit its efficiency in wetland classification (Pope et al. 1994, 1997; Hess et al. 1995).

In the $1990 \mathrm{~s}, \phi_{\mathrm{HH}}-\phi_{\mathrm{VV}}$ was among the most used polarimetric parameters for natural target characterisation (Ulaby and Elachi 1990; Touzi et al. 2004). Currently, target scattering decomposition has become the standard method for the extraction of natural target geophysical parameters from polarimetric SAR data (Touzi et al. 2004; Boerner et al. 1998). The objective of incoherent target scattering decomposition (ICTD) theory is to express natural target average scattering as the sum of independent elements in order to associate a physical mechanism with each component (Cloude 1988; Van Zyl 1992). Several techniques have been proposed during the past two decades to decompose the scattering average covariance matrix. The first class (Van Zyl 1992; Touzi 2007a; Touzi and Shimada 2009) is based on the eigenvector-based decomposition (Cloude 1988) introduced by Cloude in the context of radar imaging. The latter (Cloude 1988) was an extension of Wiener's characteristic decomposition of wave coherence matrix to target coherency matrix (Touzi and Shimada 2009; Wiener 1930). The 
characteristic decomposition of the averaged target coherency matrix (Cloude 1988) permits the characterisation of global target scattering with two parameters, the CloudePottier "averaged" scattering type $(\alpha)$ and the entropy $(H)$. This served as the basis of the very popular Cloude-Pottier $\alpha /$ $H$ classification (Cloude and Pottier 1997). The second class corresponds to model-based decompositions (Freeman and Durden 1998; Yamaguchi et al. 2005; van Zyl et al. 2011), which are based on Freeman's basic idea (Freeman and Durden 1998), supposing that target observed scattering can be modelled as the linear sum of scattering that can be represented by models of the physical scattering process. Freeman's decomposition assumes that target scattering can be modelled as the linear sum of surface, double-bounce and volume scattering (Freeman and Durden 1998).

In this study, the Touzi decomposition (Touzi 2007a; Touzi et al. 2007) will be assessed for peatland characterisation using L-band polarimetric ALOS data. This method, which exploits Cloude's eigenvector-based decomposition (Freeman and Durden 1998), permits a highresolution decomposition of target scattering in terms of roll-invariant and unique target characteristics (Touzi 2007a; Touzi et al. 2009). In contrast to Cloude-Pottier ICTD (Cloude and Pottier 1997), the new decomposition uses a complex entity, $\left(\alpha_{s}, \phi_{\alpha s}\right)$ for an unambiguous description of target scattering type (Touzi et al. 2009; Touzi 2007a). The information provided by both the scattering type magnitude $\alpha_{s}$ and phase $\phi_{\alpha s}$ has been shown to be important for wetland classification (Touzi et al. 2007, 2009). The analysis of C-band SAR data collected with the Convair-580 over the RAMSAR Mer Bleue peatland, near Ottawa, Canada, permits the demonstration of the unique potential of the scattering type phase, $\phi_{\alpha s}$, for bog-fen discrimination (Touzi et al. 2007, 2009). These two classes could not be separated using the multi-polarisation ( $\mathrm{HH}, \mathrm{HV}, \mathrm{VV})$ intensity or the $\mathrm{HH}-\mathrm{VV}$ phase difference information (Touzi et al. 2007, 2009). These very promising results obtained with the scattering type phase are confirmed in the following using polarimetric L-band ALOS data collected over boreal and subarctic peatlands (Touzi and Gosselin 2010). We will show in the following that the phase of the dominant scattering type $\phi_{\alpha s}$ is sensitive to peatland subsurface water flow variations, and this permits an efficient discrimination of bog from poor fens. The use of the additional information provided by the scattering type magnitude $\alpha_{s}$ is also required for effective wetland classification.

\subsubsection{Experimental Results}

Test sites and corresponding radar and validation data sets selected for subarctic peatland characterisation and monitoring are summarised in Table 3.9 and further described in the Appendix.

\subsection{La Baie des Mines}

\section{Peatland Hydrology Characteristics for Bog-Fen}

Discrimination

Open (sedge and shrub) bogs and poor (sedge) fens are regrouped in the peatland class of Fig. 9.1a. Traditional definitions of peatlands denote that a minimum peat layer of $30-40 \mathrm{~cm}$ is needed for an ecosystem to be considered as a peatland (Ingram 1982; Zoltai and Vitt 1995). Both bogs and fens in the peatland study site satisfy this criterion with peat thickness varying from $1 \mathrm{~m}$ to $3 \mathrm{~m}$ according to the field data. Shrub bog, which is the dominant class, has a complete ground cover of sphagnum moss with a shrub canopy dominated by ericaceous shrubs (such as Labrador tea). Poor fen areas contain vegetation that is composed primarily of sedges and an understory of sphagnum mosses. Since bog and fen vegetation can hardly be discriminated by optic and SAR sensors, their different hydrological properties can be used to discriminate them, as demonstrated in the following. In particular, the depth of peatland subsurface water will be shown to be a key parameter for bog-fen discrimination. A bog consists of two layers: one is the upper thin layer about $45 \mathrm{~cm}$ deep, known as acrotelm, through which rain sinks rapidly. Below the acrotelm, there is a much thicker layer of peat, the catotelm, of dramatically reduced hydraulic conductivity and through which water movement is very slow, which is typically less than $1 \mathrm{~m}$ /day (Ingram 1982; Zoltai and Vitt 1995; Fraser et al. 2001). Unlike bogs, a poor fen peat has a higher capacity of water retention, and water moves slowly through the fen. The field measurements in La Baie des Mines site reveal a water table $20 \mathrm{~cm}$ below the poor fen peat surface, whereas the bog subsurface water lies on the bottom of the acrotelm at about $40 \mathrm{~cm}$ below the peat surface. This is in agreement with other studies, which show that bog water remains generally $30-40 \mathrm{~cm}$ below the peat

Table 3.9 Test sites and corresponding radar and validation data selected for the generation of showcases on subarctic peatland characterisation

\begin{tabular}{l|l|l}
\hline Application/product & Test site - radar data & Reference data \\
\hline Subarctic peatland characterisation & La Baie des Mines Peatland, Quebec, Canada & Classification based on Ikonos images and forest inventory \\
\cline { 2 - 3 } & ALOS-PALSAR, 10/11/2006, 13/05/2007 & Fieldwork data \\
\cline { 2 - 3 } & Wapusk National Park, Manitoba, Canada & Brook's classification (Brook and Kenkel 2002) \\
\cline { 2 - 3 } & ALOS-PALSAR, 08/06/2010, 24/07/2010 & Landsat-5 images, 18/07/2010, 04/09/2010 \\
\hline
\end{tabular}




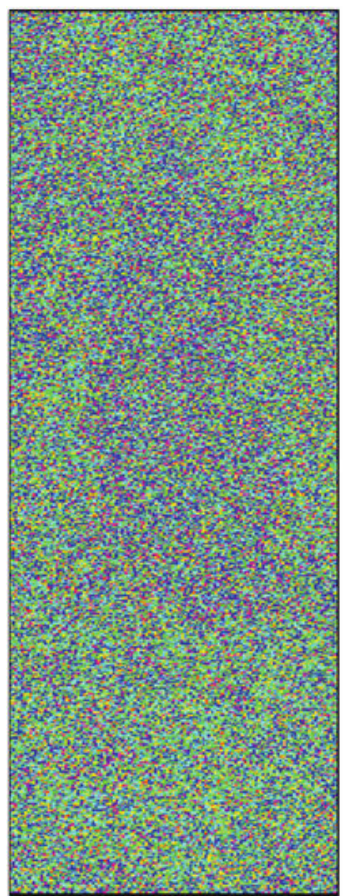

(a)

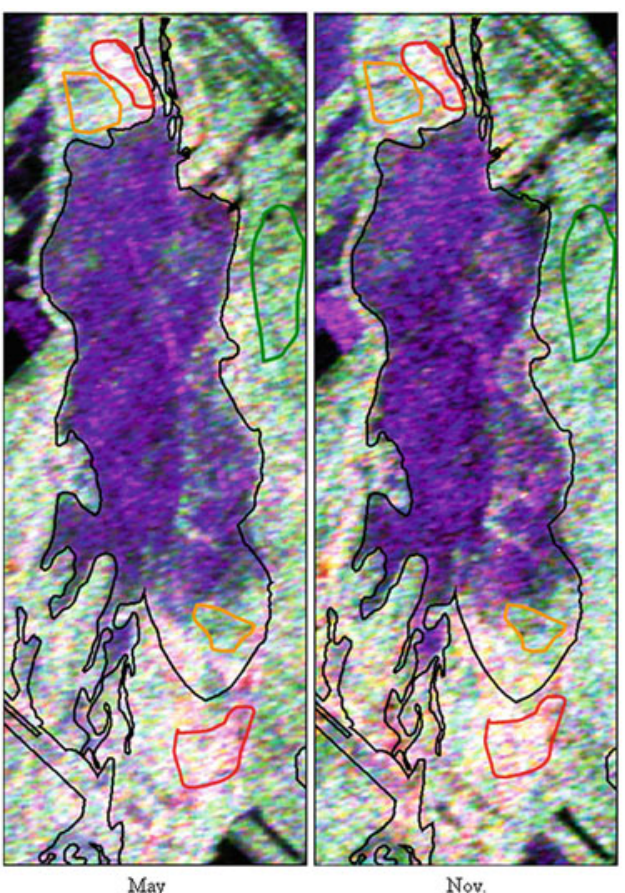

(b)

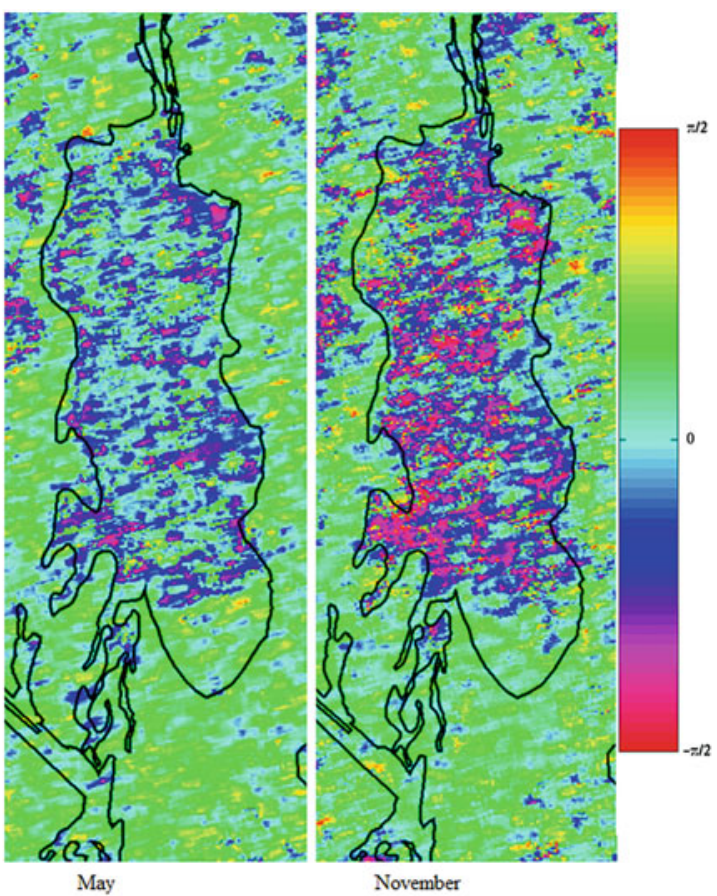

(c)

Fig. 3.31 La Baie des Mines Peatland. (a) 1-look scattering type phase (May). (b) Multi-polarisation images for May and November acquisitions (HH in red, $\mathrm{HV}$ in green and VV in blue). Wetland samples

are delineated: swamp (red), upland forest (green), treed bog (brown), open peatland (black). (c) Scattering type phase images

surface (at the catotelm layer) (Ingram 1982; Touzi et al. 2007; Fraser et al. 2001)).

\section{Application of the Touzi Decomposition to Polarimetric ALOS Data: Required Processing Window Size for Unbiased ICTD}

As mentioned above, our decomposition may be considered as an extension of Kennaugh-Huynen decomposition, which solves for con-eigenvalue phase ambiguities and can be applied for the decomposition of both coherent (presented in terms of the $[S]$ matrix) and partially coherent (presented in terms of the coherency $[T]$ matrix) target scattering. Since bog scattering might be highly coherent, the application of the decomposition under coherent conditions (1-look image) should preserve the spatial resolution. In that case, the parameters provided by the Touzi decomposition would be equivalent to the ones obtained with the Kennaugh-Huynen CTD after phase ambiguities removal. Under the assumption of coherent scattering, the decomposition is applied with $1 \times 1$ processing window on the May acquisition. Figure 3.31a presents the 1-look scattering type $\phi_{\alpha s}$. As can be noted, the phase is random because of the presence of speckle, and no useful information can be extracted. The fact that each resolution cell is not dominated by a single coherent scatterer does not permit the generation of a meaningful scattering decomposition using the CTD. We have previously shown that a processing window with a minimum of
60 independent samples is required for unbiased estimation of the ICTD parameters (Touzi 2007b). A 3-look image is firstly generated in Mueller matrix with a square pixel by replacing each pixel's Mueller matrix with the Mueller matrix averaged over the 3 pixel azimuth segment centred on the pixel. The ICTD is then applied to the 3-look image using a $7 \times 7$ processing window. The decomposition is applied under the target reciprocity assumption, and the cross-polarisation magnitude is taken as the average of $\mathrm{HV}$ and VH magnitude to increase the signal-to-noise ratio of $3 \mathrm{~dB} \mathrm{HV}=e^{j \phi_{H V}}(|\mathrm{HV}|+|\mathrm{VH}|) / 2$ (where $\phi_{\mathrm{HV}}$ is the phase of $\mathrm{HV}$ ).

\section{Analysis of the ALOS Acquisitions}

The May acquisition, in dry conditions, provides the most suitable data set for wetland classification. The dominant scattering parameters are generated with a processing window that includes more than 60 independent samples. A colour wheel with equally spaced bins between $-\pi / 2$ and $\pi /$ 2 is used to represent the scattering type phase $\phi_{\alpha s 1}$ of the dominant scattering. $\phi_{\alpha s 1}$ image of the May acquisition is presented in Fig. 3.31c, and the scattering type magnitude $\alpha_{s 1}$ image is presented in Fig. 3.32a. The classification and the field data are used to compute the statistics of the scattering type parameters and $\sigma^{0}$ for the $\mathrm{HH}, \mathrm{VV}$ and $\mathrm{VV}$ polarisation, for the various wetland classes: (poor) fen, open (sedge and shrub) bog, treed bog, swamp and upland forests. Table 3.10 
presents for each class the average and variation of each parameter for each class using the samples outlined in Fig. 3.31b. Analysis of Figs. 3.31c and 3.32a and the dominant scattering type statistics of Table 3.10 lead to the following conclusions:

1. $\phi_{\alpha s}$ permits a clear separation of poor fen $\left(\phi_{\alpha s}\right.$ about $\left.-60^{\circ}\right)$ from open bog $\left(\right.$ about $\left.-18^{\circ}\right)$. $\phi_{\alpha s}$ has similar values (about $30^{\circ}$ ) for the various treed areas: swamp, treed bog and upland forests.

2. Even though the scattering magnitude $\alpha_{s 1}$ cannot separate poor fen from bogs, $\alpha_{s 1}$ discriminates well the swamp class from the upland forest and treed bog. $\alpha_{s 1}$ is very efficient in detecting the quasi-dipole scattering $\left(\alpha_{s 1}=40^{\circ}\right)$ due to the wave interactions of water and trees in the swamp. The fact that the contribution of the trihedral and dihedral scattering is not added in phase $\left(\phi_{\alpha s}=31^{\circ}\right)$ leads to a quasi-dipole scattering instead of the perfect-dipole scattering (with $\alpha_{s 1}=45^{\circ}$ and $\phi_{\alpha s}=0^{\circ}$ ) (Touzi et al. 2007).

3. It is worth noting that the swamp quasi-dipole scattering can also be detected with $\mathrm{HH}$ (much larger than VV and HV) as can be expected.
Therefore, we can conclude that the information provided by both the scattering type magnitude and phase is required for wetland characterisation. To validate the unique potential of the scattering type phase for discrimination of poor fens from bogs, in situ field measurements were collected. The areas that appear in pink $\left(\phi_{\alpha s 1}\right.$ about $\left.-60^{\circ}\right)$ on Fig. 3.31c were effectively identified as fens. They are dominated by herbs, and the water level lies between 10 and $20 \mathrm{~cm}$ under the peat surface. The sites that look dark blue on Fig. 3.31c were identified as bogs. They are dominated by Labrador tea and a very thick peat (from $1.6 \mathrm{~m}$ to $3 \mathrm{~m}$ deep). The water table is much deeper $(40-50 \mathrm{~cm}$ under the peat surface) in the bog samples, as expected according to hydrologic properties of bogs (Fraser et al. 2001).

\section{Peatland Subsurface Water Flow Monitoring Using \\ Polarimetric May and November ALOS Acquisitions: \\ Multi-polarisation Versus Polarimetric Information}

The spring acquisition took place under dry conditions; no rain for 14 days and warm weather with a temperature between $10^{\circ}$ and $20^{\circ}$. The fall image was collected under cool weather (with a temperature below $5^{\circ}$ ) and wet

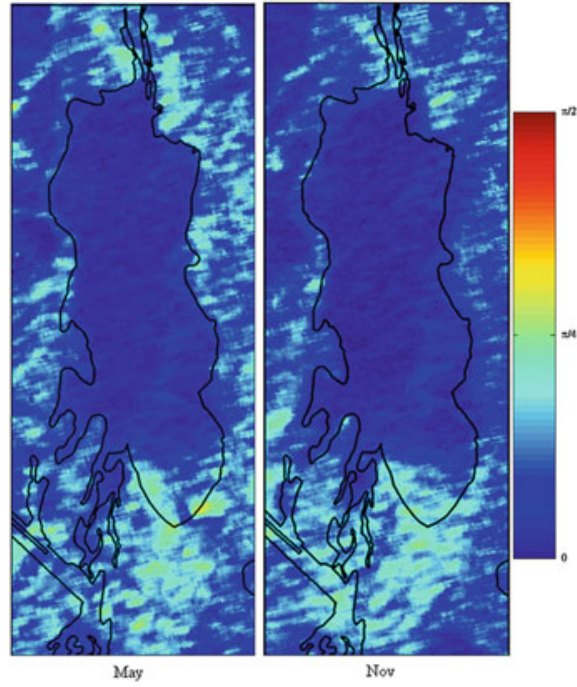

(a)

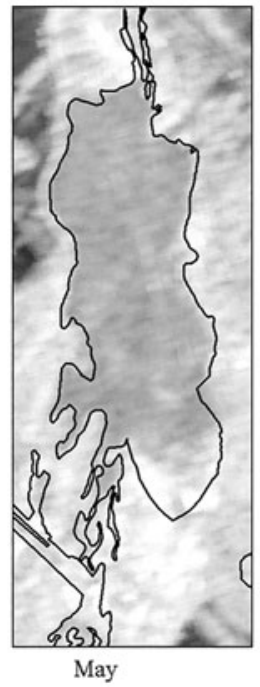

(b)

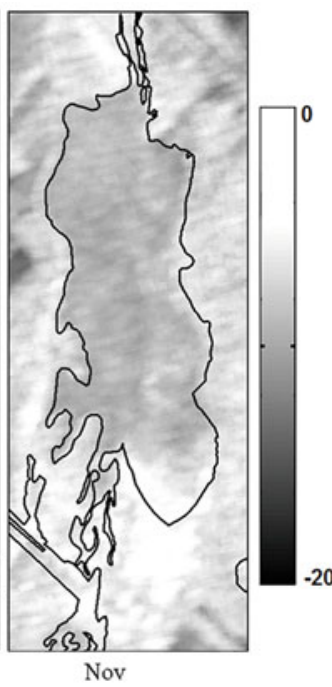

b)

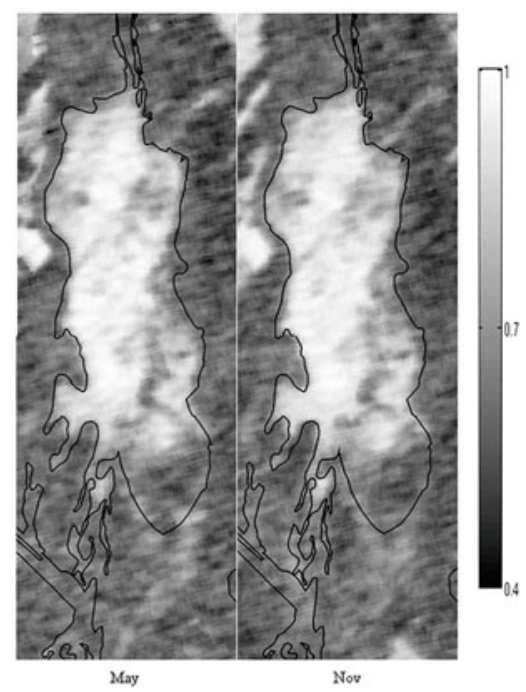

(c)

Fig. 3.32 May and November images: (a) $\alpha_{s 1} ;$ (b) $m_{1} ;$ (c) $\lambda_{1}$

Table 3.10 Scattering type parameters estimates and HH, HV and VV for May acquisition

\begin{tabular}{|c|c|c|c|c|c|}
\hline Class & $\phi_{\alpha s 1} \mathrm{Z}(\mathrm{deg})$ & $\alpha_{s 1} \mathrm{Z}(\mathrm{deg})$ & $\mathrm{HH}(\mathrm{dB})$ & VV (dB) & $\mathrm{HV}(\mathrm{dB})$ \\
\hline Open bog & $-18^{\circ} \pm 3^{\circ}$ & $6^{\circ} \pm 3^{\circ}$ & $-6.8 \pm 1$ & $-7.1 \pm 1$ & $-17.5 \pm 1$ \\
\hline Poor fen & $-60^{\circ} \pm 3^{\circ}$ & $6^{\circ} \pm 3^{\circ}$ & $-6.8 \pm 1$ & $-7.2 \pm 1$ & $-17 \pm 1$ \\
\hline Treed bog & $30^{\circ} \pm 3^{\circ}$ & $15^{\circ} \pm 3^{\circ}$ & $-5 \pm 1$ & $-6.5 \pm 1$ & $-12 \pm 1$ \\
\hline Swamp & $31^{\circ} \pm 3^{\circ}$ & $40^{\circ} \pm 3^{\circ}$ & $-2.5 \pm 1$ & $-5.6 \pm 1$ & $-11 \pm 1$ \\
\hline Forest & $30^{\circ} \pm 3^{\circ}$ & $25^{\circ} \pm 3^{\circ}$ & $-5 \pm 1$ & $-6.5 \pm 1$ & $-12 \pm 1$ \\
\hline
\end{tabular}


conditions; about $10 \mathrm{~mm}$ rain accumulated during the 2 days that precede the acquisition. Since the L-band ALOS penetrating wave is sensitive to wetland groundwater conditions, we should expect a significant change in radar backscattering at $\mathrm{HH}, \mathrm{HV}$ and $\mathrm{VV}$ polarisations. Figure $3.31 \mathrm{~b}$ presents the composite colour of $\mathrm{HH}, \mathrm{HV}$ and $\mathrm{VV}$ for both acquisitions. Water-level change can be noted in the swamps outlined in Fig. 3.31b. However, no change can be detected in the open peatland. The multi-polarisation information looks similar for the two acquisitions even though we might expect significant changes in the peatland subsurface water flow between the dry and wet acquisitions. These changes cannot also be detected using the dominant scattering type parameters $\alpha_{s 1}, m_{1}$ and $\lambda_{1}$ as can be noted in Fig. 3.32. Analysis of all the other intensity parameters (span, $\lambda_{i}, m_{i}, i=1, \ldots, 3$ ) and Cloude-Pottier's parameters ( $\alpha$, entropy and anisotropy) leads to similar conclusions. None of them are sensitive to water flow changes beneath the peat surface.

Like in (Touzi 2007a), the scattering type phase $\phi_{\alpha s 1}$ seems to be the only polarimetric parameter that can detect peatland subsurface water flow changes, as can be noted in Fig. 3.31c. Major changes (pink to dark blue) in $\phi_{\alpha s}$ can be noted when the phase images of May and November are compared. These significant variations (larger than $40^{\circ}$ ) represent the significant variations of the water flow beneath the peat surface between the dry and wet (May-November) peatland conditions. The pink colour, which indicates the presence of subsurface water, is dominant in the November acquisition. The latter acquisition took place shortly after the rain stopped, and this does not give enough time for the rain water to sink deeply into the acrotelm. This makes fen-bog discrimination difficult since the L-band wave reaches the subsurface water in both fens and bogs. Bog-fen discrimination is easier with the May data set collected under dry conditions, as discussed previously.

It is worth noting that similar observations regarding the higher sensitivity of the radar signal phase to subsurface features in comparison with the detected intensity were brought out by Lasne et al. (2004). Using the airborne L-band RAMSES SAR, they showed that the phase difference $\phi_{\mathrm{HH}}-\phi_{\mathrm{VV}}$ permits the detection of pale soils buried in a bare sandy area near Bordeaux (France), at a depth greater than $5.2 \mathrm{~m}$. These pale soils cannot be detected with the radar signal intensity at $\mathrm{HH}, \mathrm{HV}$ or VV polarisation, which is only sensitive to the presence of pale soil that is not deeper than $3.5 \mathrm{~m}$ (Lasne et al. 2004). One might expect that the phase difference $\phi_{\mathrm{HH}}-\phi_{\mathrm{VV}}$ of the like-polarisations is also sensitive to the peatland subsurface water flow. The phase $\phi_{\mathrm{HH}}-\phi_{\mathrm{VV}}$ and the Pauli phase difference, i.e. phase of $(\mathrm{HH}-\mathrm{VV}) /(\mathrm{HH}+\mathrm{VV})$, are presented in Fig. 3.33a, b, respectively. As can be seen, both $\mathrm{HH}-\mathrm{VV}$ phase difference

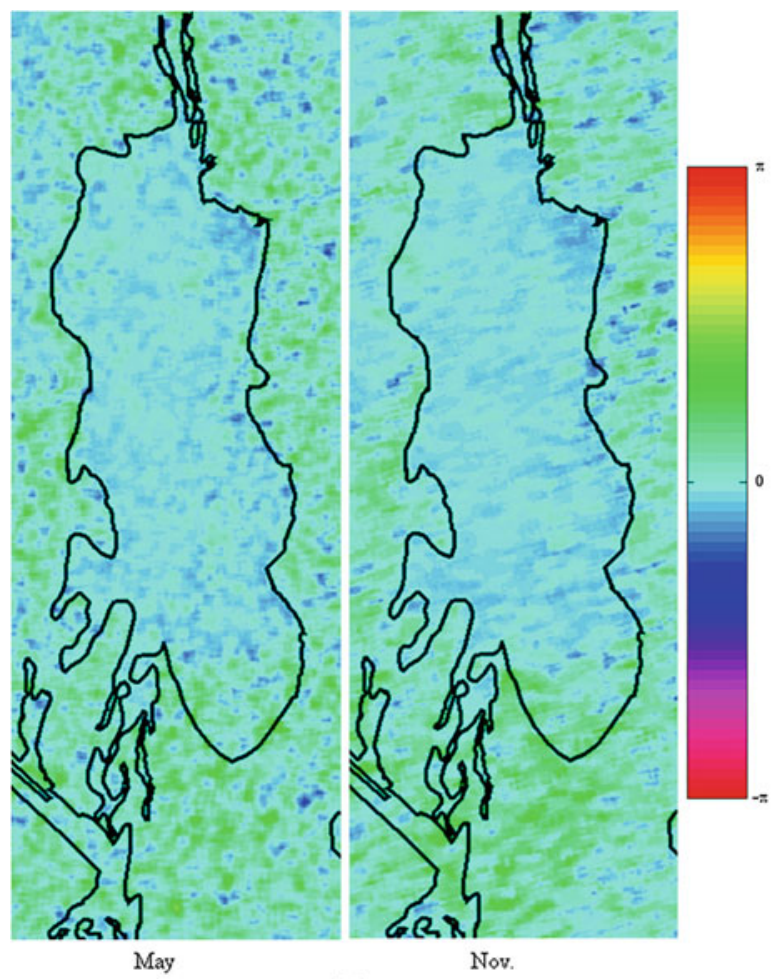

(a)

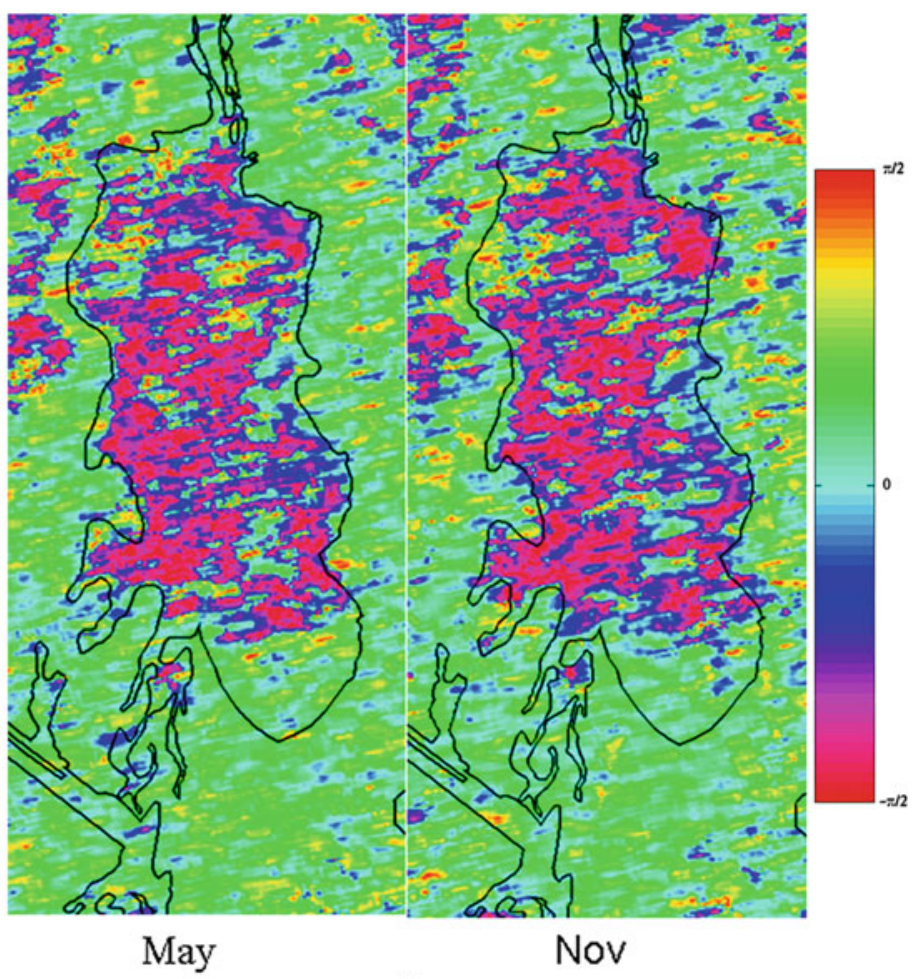

(b)

Fig. 3.33 Phase and orientation images: (a) $\phi_{\mathrm{HH}}-\phi_{\mathrm{VV}}$; (b) Pauli phase difference 
and the Pauli phase are not able to detect peatland subsurface water flow changes. This is in agreement with the results of a previous study on the Mer Bleue wetland (Touzi 2007a). The optimum phase $\phi_{\alpha s 1}$, which is sensitive to peatland subsurface water flow, is generated with a non-zero orientation angle in a polarisation basis different from the conventional $(\mathrm{H}-\mathrm{V})$ linear-polarisation basis. The Kennaugh-Huynen diagonalisation of the coherent dominant scattering matrix leads to the generation of the maximum polarisation, whose phase $\phi_{\alpha s 1}$ permits the detection of peatland subsurface water flow. It is worth noting that such promising results obtained with $\phi_{\alpha s 1}$ could only be derived, thanks to the excellent quality of ALOS HV measurement in terms of calibration and high signal-to-noise HV measurement (Touzi and Shimada 2009). An accurate measurement of HV is required to generate the optimum polarisation whose scattering type phase $\phi_{\alpha s 1}$ is sensitive to the water beneath the peat surface. This makes this unique application suitable with only fully polarimetric SAR. The reconstruction of HV from dualpolarisation RH-RV Compact (Souyris et al. 2005; Raney 2007) SAR measurements (using Souyris's method (Souyris et al. 2005)) does not permit the generation of the optimum $\phi_{\alpha s}$ required for peatland subsurface water flow monitoring, as demonstrated in (Touzi 2013).

\subsection{Wapusk National Park}

The area of study is dominated mainly by a sedge bulrush poor fen (dark orange) in an area that includes lichen melt pond bog, peat plateau bog, spruce bog and sedge-rich fens. During the spring and summer active layer melting season, we should expect important changes in the peat subsurface water flow in the fen class. A thermistor cable was installed in a pond bog (Dyke and Wendy 2010). The active layer thickness was $13 \mathrm{~cm}$ in June at the start of the melting season, $27 \mathrm{~cm}$ in July and more than $80 \mathrm{~cm}$ in September. Figure 3.34 presents the colour composite of HH (red), HV (green) and VV (blue) for the June and July acquisitions, respectively. As can be noted, the radiometric information provided by the multi-polarisation information cannot detect any change in the peatland hydrology between the June start of the melting season and the late part of July.

Our ICTD is applied to the ALOS images as described in Sect. 9.5.1.3, and the various ICTD parameters are analysed. Again, the scattering type phase $\phi_{\alpha s}$ of Fig. 3.35 is the only target scattering decomposition parameter that has revealed peatland subsurface flow variations. Major changes in $\phi_{\alpha s}$ can be noted when the phase images of early June and late July are compared. The bulrush sedge poor fen area, in particular, outlined in Fig. 3.35a (and denoted (2)) shows significant changes. This area is dominated in the June image by the pink colour, as might be expected. $\phi_{\alpha s}$ is sensitive to the fen shallow subsurface water lying on the permafrost surface (about $13 \mathrm{~cm}$ deep). In July, the fen is still irrigated by shallow subsurface water that can be detected (in pink) by the L-band wave, whereas a large part of the fen subsurface water either has been evaporated or was too deep (more than $25 \mathrm{~cm}$ ) to be detected by $\phi_{\alpha s 1}$. No change can be noted on the bogs (in dark blue) between June and July acquisition, and the recent field trip allowed us to explain the phase behaviours in these bog areas, as discussed in the following.

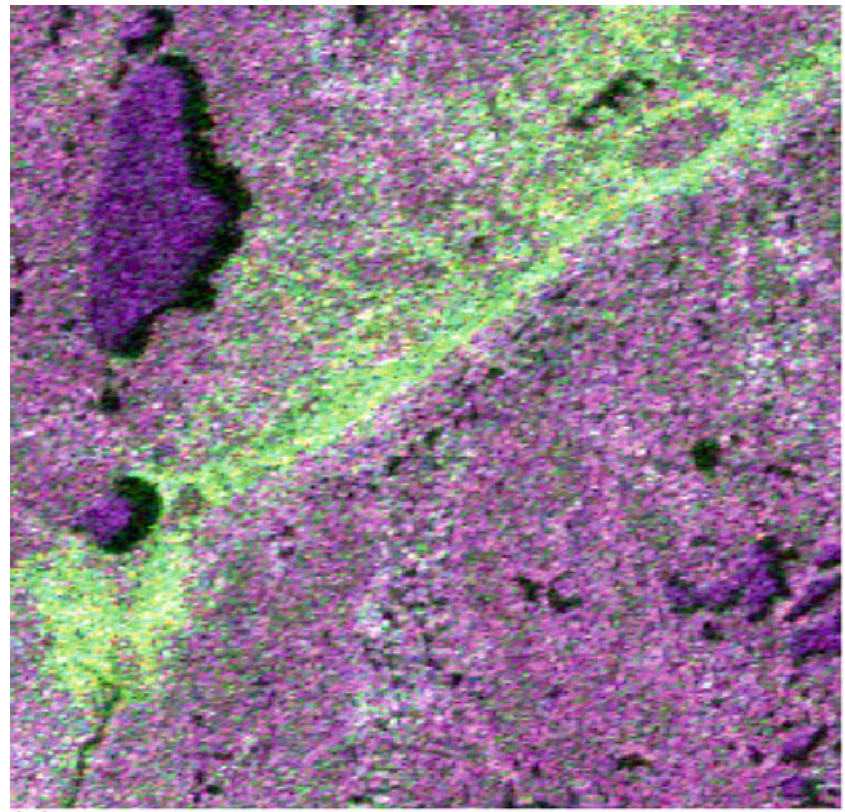

(a)

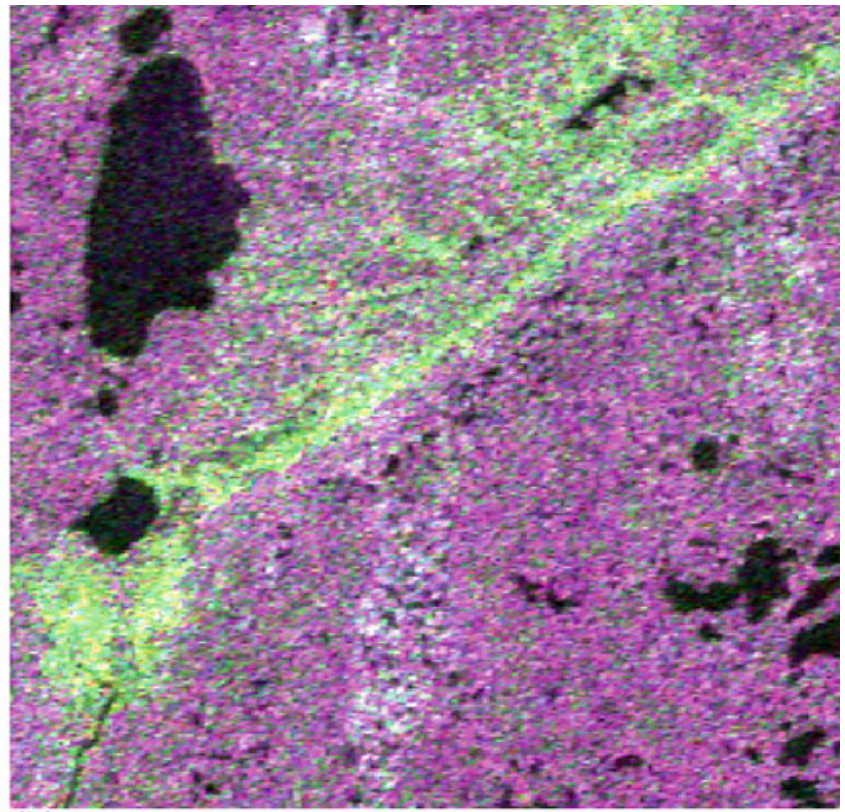

(b)

Fig. 3.34 Multi-polarisation (HH, HV, VV) images for the bog-fen peatland in Wapusk National Park (June and July 2010) 


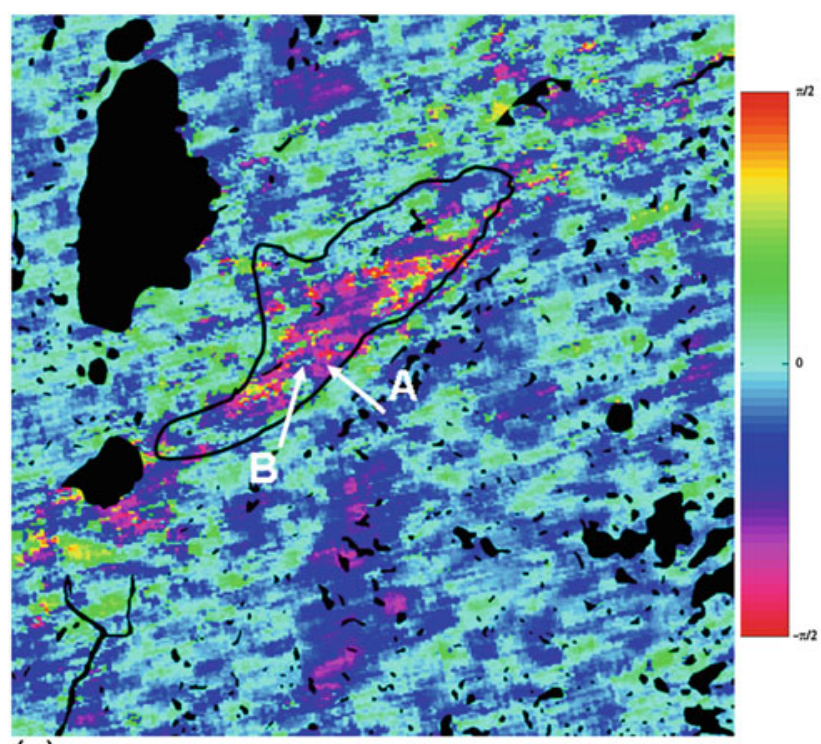

(a)

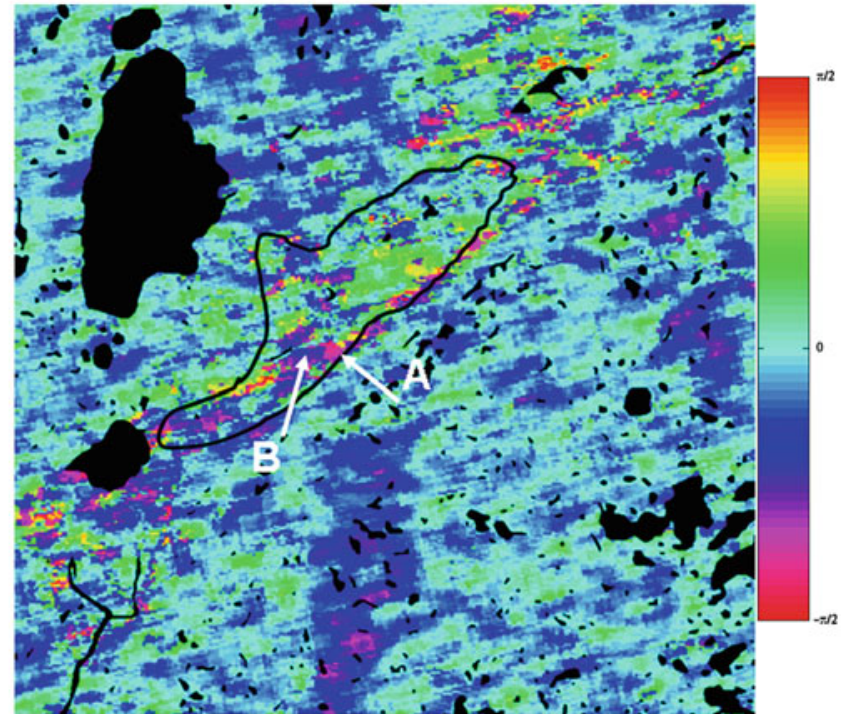

(b)

Fig. 3.35 Scattering type phase image for the bog-fen peatland in Wapusk National Park: (a) June; (b) July

In the first week of July 2012, additional in situ field measurements were collected in the Wapusk National Park peatland for further validation of the results above. Helicopter was used to visit various samples in the areas 1 and 2 outlined in Fig. 3.35a. These areas were assigned to the sedge bulrush fen and lichen melt pond bog classes, respectively, according to Brook's classification. Water-level measurements, vegetation species characterisation, active layer and peat thickness were collected in the various sites. The average of active layer thickness in the bogs and fens visited during the field trip in July is deeper than $25 \mathrm{~cm}$. Areas in pink were identified as fens with herb vegetation and water level 10 to $20 \mathrm{~cm}$ beneath the peat surface. Samples in dark blue were identified as bogs. No water was detected under the peat surface or at the frozen ground about $25 \mathrm{~cm}$ under the peat surface. The water coming to bogs from precipitations has evaporated, while the fens are continuously irrigated by subsurface and runoff water. The absence of subsurface water in bogs should explain the stability of the phase (dark blue) between the June and July acquisitions, in particular in the area (1) on the bottom of in Fig. 3.35. This area (1) outlines a fen site A (pink in the phase image) and a bog site B (dark blue in the phase image) that were visited. Pictures of the corresponding peat samples are shown in Fig. 3.36. While no water can be seen under the bog peat surface (at the frozen ground interface), the water level at the shrub fen was about $15 \mathrm{~cm}$ under the peat surface, as can be seen on the peat sample pictures of Fig. 3.36c, d. These observations confirm the promising potential of $\phi_{\alpha s}$ for bog-fen discrimination. Since no water lies beneath bog peat surface, $\phi_{\alpha s}$ can easily identify poor fens of shallow subsurface water. The sensitivity of polarimetric L-band scattering phase to peatland subsurface water should provide an efficient tool for monitoring bog-fen transformations.

\subsubsection{Discussion on the Role of Polarimetry, on the Maturity of the Application and Conclusions}

This study confirms that the scattering type phase $\phi_{\alpha s}$ extracted from fully polarimetric L-band ALOS data can reveal the seasonal changes in poor fen subsurface water flow and permits the separation of poor fen from shrub bogs. Such information cannot be obtained with the multi-polarisation $\mathrm{HH}, \mathrm{HV}$ and $\mathrm{VV}$ intensities nor with the conventional polarimetric decomposition parameters such as the Cloude-Pottier $\alpha$, the entropy and the extrema of the intensities provided by the coherency eigenvalues. $\phi_{\alpha s}$, which cannot detect deep ( $45 \mathrm{~cm}$ below the peat surface) water in a boreal bog, seems to be more sensitive to the shallower water $(10-20 \mathrm{~cm})$ beneath the surface in fen, and this makes possible the separation of poor fens from shrub bogs. These results have recently been confirmed in a boreal peatland in the Athabasca region in the context of an investigation on the long-term monitoring of oil sand exploration on surrounding peatland. The use of polarimetric L-band SAR and $\phi_{\alpha s}$ could also be very promising for the detection of new fens in subarctic bogs. Recent field work in the Wapusk National Park peatlands revealed the absence of water (on the surface of the permafrost) beneath the peat bog surface. Fens, which are continuously irrigated by subsurface water 10-20 cm deep, could be clearly identified with $\phi_{\alpha s}$. Even though we are convinced that further experiments are needed to validate these results with other peatland sites, the results obtained so far look very promising for the operational 
a
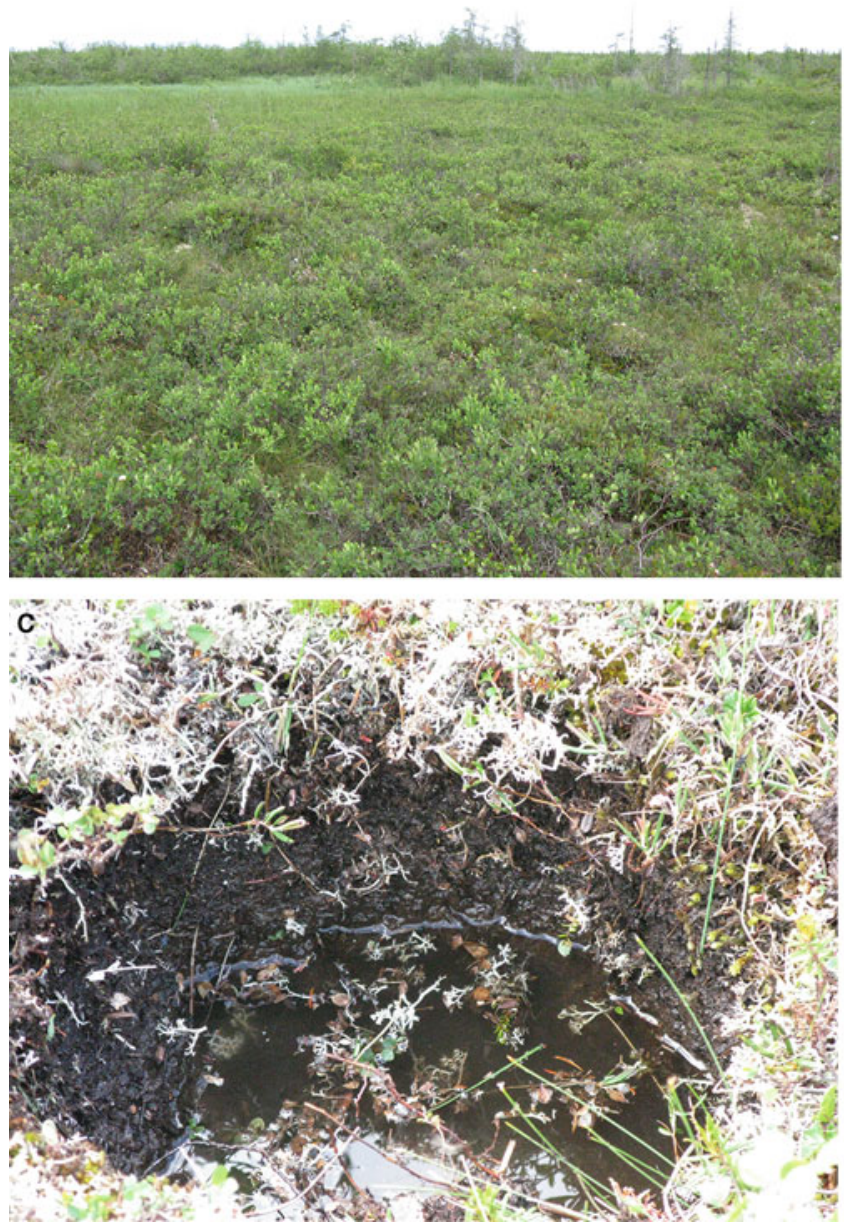

b
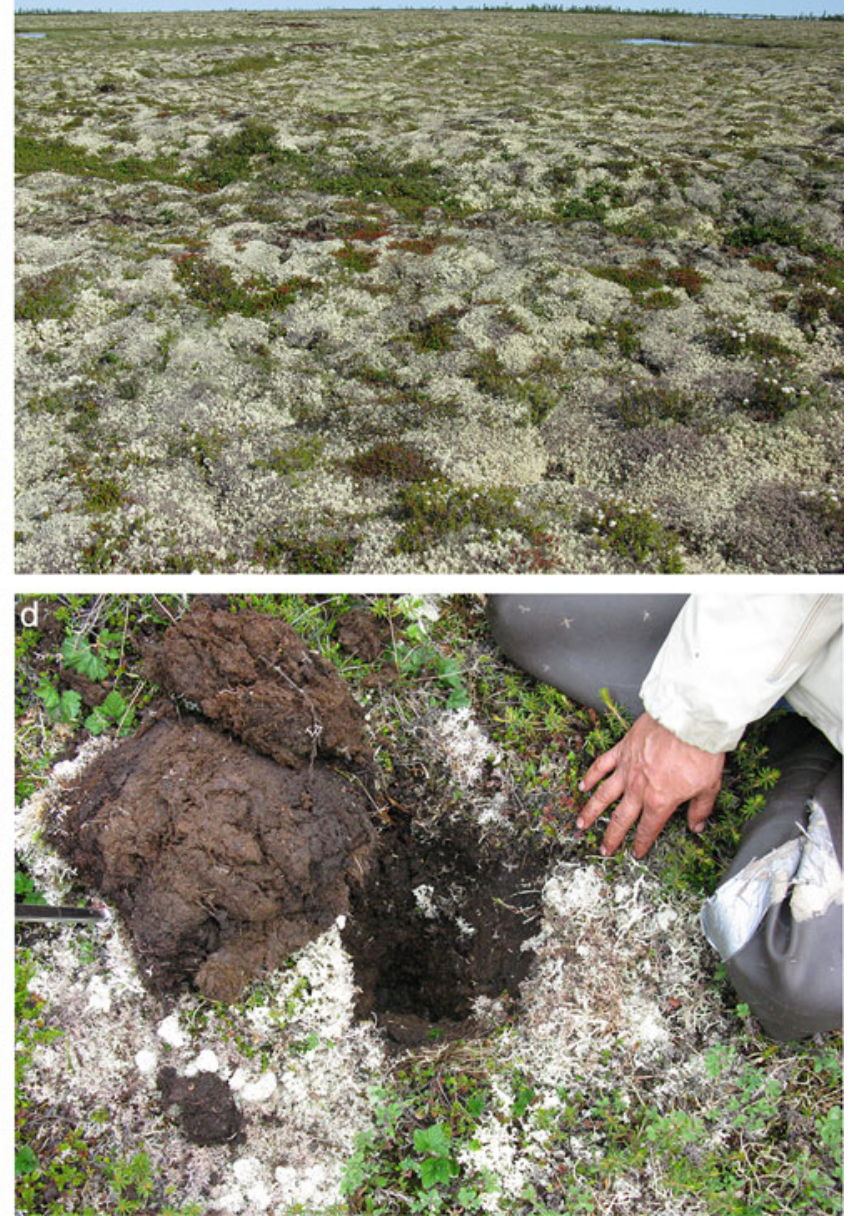

Fig. 3.36 Field data collection. (a) Sedge fen A. (c) Water-level picture in sedge fen A. (b) Open bog B. (d) Water-level picture in bog B; no water at the frozen ground interface

use of the upcoming polarimetric L-band SAR satellite missions, and ALOS2 in particular, as an essential source of information for mapping peatlands and monitoring bog to fen transformations. Operational windows of polarimetric L-band SAR acquisitions are required from the spring runoff water to the fall season, and over a long period of time, for efficient peatland monitoring.

\subsubsection{Acknowledgement}

The authors would like to thank S. Kowalchuk, M. Gibbons, J. Larkin and H. Stewart from the Wapusk National Park for their help in organising and carrying out field data collection. S. Nedelcu and K. Omari from CCRS are thanked for their help in data processing. We would also like to thank JAXA for having provided the ALOS-PALSAR data under the ALOS-PI project and the Canadian Space Agency for having partially funded the present study under the Government Related Initiative Program.

\subsection{Monitoring Change Detection Produced by Tsunamis and Earthquakes by Using a Fully Polarimetric Model-Based Decomposition}

\subsubsection{Introduction, Motivation and Literature Review}

Natural disasters occur frequently, causing significant loss of life and leading to major geo-/bio-environmental and socioeconomic costs. Therefore, the monitoring of the disaster damages over the globe is an urgent need. It is quite difficult to obtain an immediate response of large-scale earthquakes and tsunami disaster areas by ground survey methods. Although the ground survey is accurate, it is also highly time-consuming and manpower extensive, and, consequently, this causes delays in assessment responses to rescue 
teams. Satellite remote sensing has great potential in the monitoring of disaster damages because of its repetitive capability and synoptic coverage. The aim of this work is to present a methodology to generate information from highresolution polarimetric SAR images to identify directly the differences or damages between pre- and post-tsunami and earthquake conditions on the affected regions. This methodology is based on scattering decomposition techniques for polarimetric data. We take the advantage of the excellent quad-polarisation data sets acquired with the Japanese Advanced Land Observing Satellite-Phase Array type L-band Synthetic Aperture Radar (ALOS-PALSAR) imaging system using its high-resolution PolSAR mode to produce colour-coded images for easily interpreting earth surface features and monitoring the earthquake and tsunami damage along the Miyagi coast affected by the 11th of March 2011 tsunami.

Assessment of earthquake and tsunami disaster damages in urban areas has been investigated based on visual interpretation or change detection methods using remotely sensed imagery. Hitherto, several methods are available to monitor earthquake and tsunami damages by using multispectral and monochromatic optical images as well as mono/dualpolarisation SAR images (Matsuoka and Koshimura 2010; Chini et al. 2009). High-resolution multispectral optical images enable direct visual interpretation of the damages and are rather straightforward and simple to interpret by users. However, optical remote sensing fails under cloudy, foggy and hazy as well as severe rainy conditions for monitoring near-real-time damage. Single-wavelength SAR images obtained from fixed single- and/or dual-polarisation sensors are independent of meteorological conditions, but are difficult to be interpreted and require tedious computational analyses for assessments. Most recently, the potential and advantages of fully polarimetric SAR data to monitor the natural disasters, including tsunami and earthquake, were demonstrated in (Yamaguchi 2012) and (Sato et al. 2012b).

Scattering power decompositions have been a research topic in radar polarimetry for the analysis of fully polarimetric synthetic aperture radar data (Freeman and Durden 1998; Yajima et al. 2008; Yamaguchi et al. 2005; 2011; Arii et al. 2011; Sato et al. 2012a; van Zyl et al. 2011; Lee and Ainsworth 2011; Touzi 2007a; Singh et al. 2012). There exist nine real independent observation parameters in the $3 \times 3$ coherency or covariance matrix with respect to the second-order statistics of polarimetric information (Freeman and Durden 1998; Yajima et al. 2008; Yamaguchi et al. 2005; Touzi 2007a). There are several decomposition methods to retrieve information from the coherency matrix. Physical scattering model-based decompositions are straightforward to interpret the final imaging result because the experimental evidence is incorporated in the model-based approach. The pioneering work of the model-based decomposition was presented by Freeman and Durden (Freeman and Durden 1998) by introducing the three-component decomposition. To date, a significant amount of research has been carried out on the model-based decomposition techniques (Arii et al. 2011; Sato et al. 2012a; Yamaguchi et al. 2011; van Zyl et al. 2011; Lee and Ainsworth 2011; Singh et al. 2012).

The original three-component decomposition was proposed by Freeman and Durden (1998) under the reflection symmetry condition, i.e. the cross-correlation between the co- and cross-polarised scattering elements are close to zero for natural distributed objects. This method decomposes the observation matrix into the surface, double bounce and volume scattering terms based on the physical scattering models and accounts for five terms out of nine independent parameters. Then, Yamaguchi et al. (2005) added a helix scattering term and proposed the four-component decomposition. Then, by using the rotation of coherency matrix, Yamaguchi et al. (2011) reduced the number of polarimetric parameters from 9 to 8. The Yamaguchi et al. (2011) method yielded better decomposition results by accounting for six parameters out of eight. The un-accounted parameters are the real and imaginary part of $T_{13}$ in the coherency matrix, and they still remained un-accounted in any of the known physical scattering model-based decompositions. Finally, a general four-component decomposition (G4U) method has been proposed recently by Singh et al. (2012) using a special unitary transformation to the rotated coherency matrix, which has been used in the existing four-component decomposition. Since unitary transformations do not change any information included in the coherency matrix, the rotated coherency matrix is transformed to eliminate the $T_{23}$ element. This four-component decomposition finally accounts for seven terms out of seven polarimetric parameters.

\subsubsection{Methodology}

Figure 3.37 shows the general four-component scattering power decomposition procedure (G4U). The number of independent parameters in the coherency matrix is reduced from 9 to 7 by the rotation and the special unitary transformations. This decomposition scheme describes the total scattering power into surface scattering power $P_{s}$, double-bounce scattering power $P_{d}$, volume scattering power $P_{v}$ from dipole and/or oriented dihedral and helix scattering power $P_{c}$. This recently developed four-component scattering power decomposition scheme also includes the complete fully polarimetric relative phase information and the extended volume scattering model for oriented dihedral structures (Singh et al. 2012) (which are the most convenient in urban areas to estimate the appropriate volume scattering as compared to threecomponent decomposition (Freeman and Durden 1998)). The decomposition starts by retrieving the helix scattering 


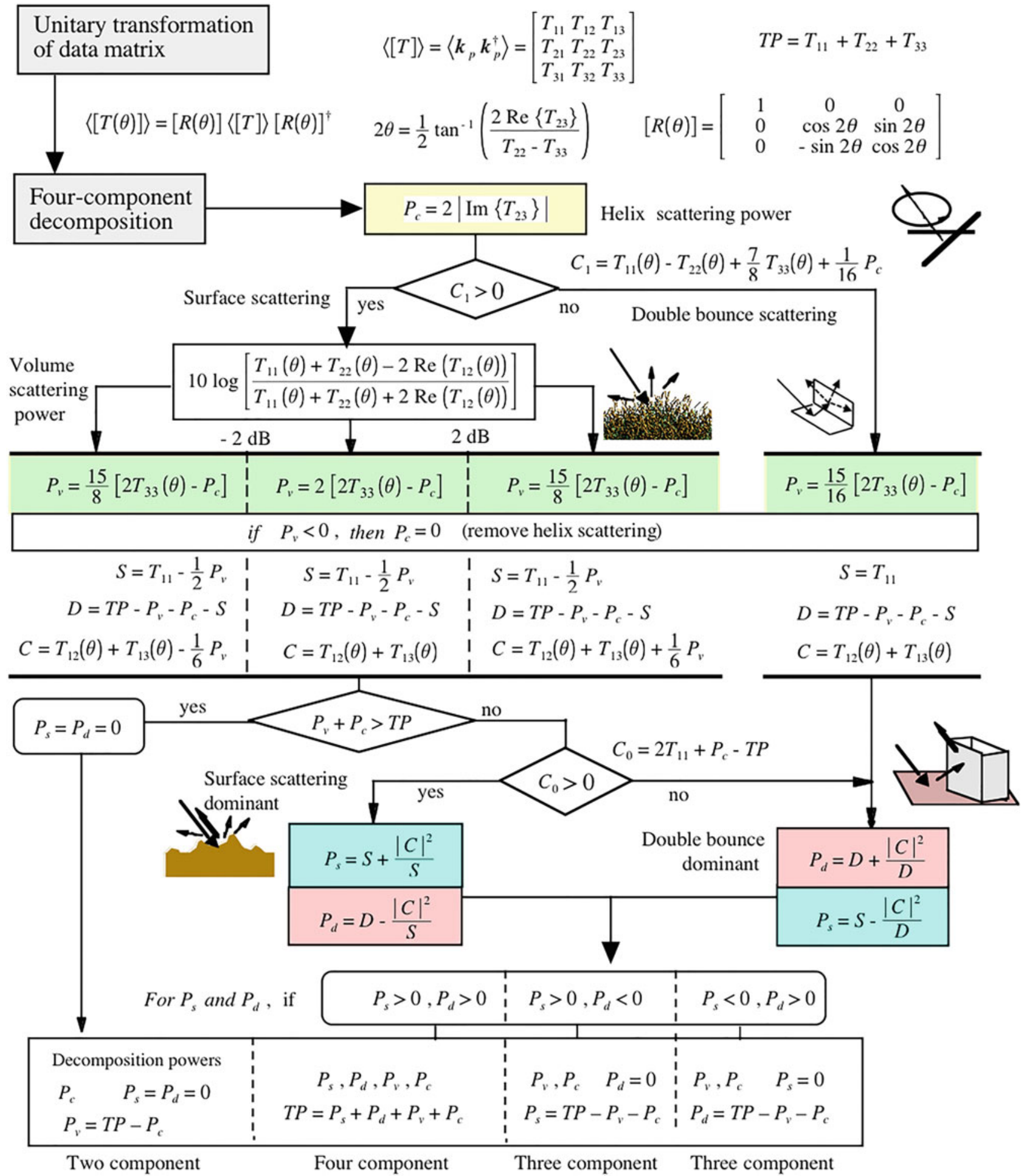

Fig. 3.37 General four-component scattering power decomposition algorithm (G4U) (Singh et al. 2012)

power. The branch condition for $\mathrm{C} 1$ is used to retrieve double-bounce scattering caused by oriented dihedrals. The second branch condition for $\mathrm{C} 0$ is to select dominant scattering mechanism. The outputs of the G4U are used to examine the disaster areas.
Colour composite images of Fig. 3.38 were generated with multi-look factors of 18 times in azimuth direction and 3 times in range direction for the PALSAR data sets in Table 3.9. After the decomposition of ALOS-PALSAR data over the earthquake/tsunami disaster site, the four scattering 

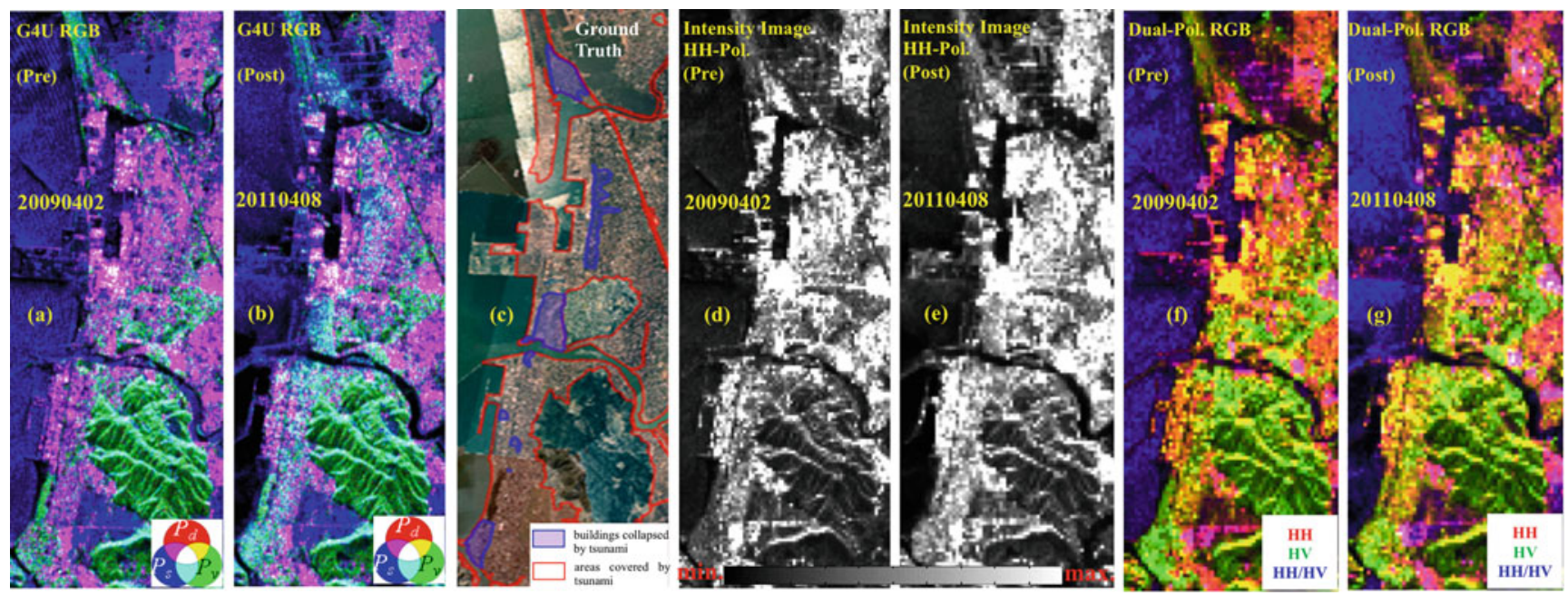

Fig. 3.38 (a) Pre- and (b) post-earthquake/tsunami G4U colour-coded images for PALSAR data. (c) Ground truth information, (d) pre- and (e) post-earthquake/tsunami HH-pol intensity images for PALSAR data and (f) pre- and (g) post-earthquake/tsunami dual-pol RGB images of

PALSAR data. In all images, the flight direction of ALOS-PALSAR is from left to right, and PALSAR illumination direction is from top to bottom

Table 3.11 Test sites and corresponding radar and validation data selected for the generation of showcases on monitoring of post-disaster effects in urban areas

\begin{tabular}{l|l|l}
\hline Application/product & Test site - radar data & Reference data \\
\hline $\begin{array}{l}\text { Monitoring of post-disaster } \\
\text { effects in the urban areas }\end{array}$ & $\begin{array}{l}\text { Ishinomaki, Miyagi } \\
\text { Prefecture, Japan }\end{array}$ & $\begin{array}{l}\text { Ground truth information provided by the Association of Japanese Geographers } \\
\text { and Geospatial Information Authority of Japan }\end{array}$ \\
\cline { 2 - 2 } & $\begin{array}{l}\text { ALOS-PALSAR data } \\
\text { set \#20090402 }\end{array}$ & \\
\cline { 1 - 2 } & $\begin{array}{l}\text { ALOS-PALSAR data } \\
\text { set \#20110408 }\end{array}$ & \\
\hline
\end{tabular}

components $\left(P_{s}, P_{d}, P_{v}\right.$ and $\left.P_{c}\right)$ were normalised by total power $(T P)$ for further analysis. A mean filter with $3 \times 3$ window size is applied on these normalised scattering power component images before analysing the images in more detail (Singh et al. 2013).

\subsubsection{Experimental Results}

We have selected parts of the coastal areas within the Miyagi Prefecture affected by the 11th of March 2011 magnitude 9.0 Honshu, Japan, earthquakes (38.322 N, 142.369 E, depth $32 \mathrm{~km}$ ) that struck off Japan's northeastern coast and triggered a historical super-tsunami. We used the ALOSPALSAR fully polarimetric, single-look complex (SLC), level 1.1 (ascending orbit) images acquired over study areas before (2nd of April 2009) and after the earthquake/tsunami (8th of April 2011) struck; see Table 3.11.

Two (pre- and post-disaster) images are processed by implementing the G4U scheme (Singh et al. 2012) to the data sets in Table 3.11. These decomposition RGB colourcoded images of 20090402 and 20110408 are presented in Fig. 3.39. Since man-made structures such as building and bridges orthogonal to radar illumination are categorised into double-bounce scatterer types in the G4U scheme, the double-bounce $\left(P_{d}\right)$ scattering component in urban area is caused by right angle scattering between building block walls and road surfaces. The volume scattering $\left(P_{v}\right)$ and surface scattering $\left(P_{s}\right)$ components are small for orthogonally illuminated man-made structures. However, damaged or collapsed urban blocks or man-made structures resulting from the earthquake/tsunami impact that do not appear to be orthogonal to radar direction, and which corresponding main scattering centre is at an oblique direction with respect to radar illumination, generate no double-bounce type response in the G4U images after the tsunami struck. Due to multiple scattering, these red areas (pre-tsunami image) turn into green (volume scattering) in post-tsunami images. In cases of buildings washed out and/or eliminated by the tsunami, these areas appear as blue (i.e. surface scattering types of the G4U scheme) in the post-tsunami images. These effects in post-tsunami images, compared to pre-tsunami images, provide a simple straightforward tool for interpreting collapsed buildings in tsunami-affected areas (Singh et al. 2013). 
The changes produced by the disaster are shown in Fig. 3.39 using the difference in the normalised scattering powers $p_{s}\left(=P_{s} / T P\right), p_{v}\left(=P_{v} / T P\right)$ and $p_{d}\left(=P_{d} / T P\right)$. Table 3.12 shows the quantitative mean statistics of the normalised difference scattering parameters $\Delta p_{s}, \Delta p_{v}, \Delta p_{d}$ and $\Delta p_{c}$ corresponding to surface, volume, double bounce and helix scattering powers, for patch A (vegetated area, Fig. 3.39), patch B (agricultural area/wiped-out houses debris-deposited area, Fig. 3.39) and patch C (urban region,
Fig. 3.39). The mean statistics including \pm standard deviation of the pixel distributions over all patches were analysed, and the basic behaviours of scattering parameters $p_{s}, p_{v}$ and $p_{d}$ were observed as follows: (1) the surface scattering is increased in tsunami-affected areas (vegetation-damaged area and wiped-out/collapsed urban block area), but it can be decreased in the areas with deposition of wiped-out houses by the retreating tsunami and for the floating houses on bay areas; (2) the volume scattering is decreased in tsunami-
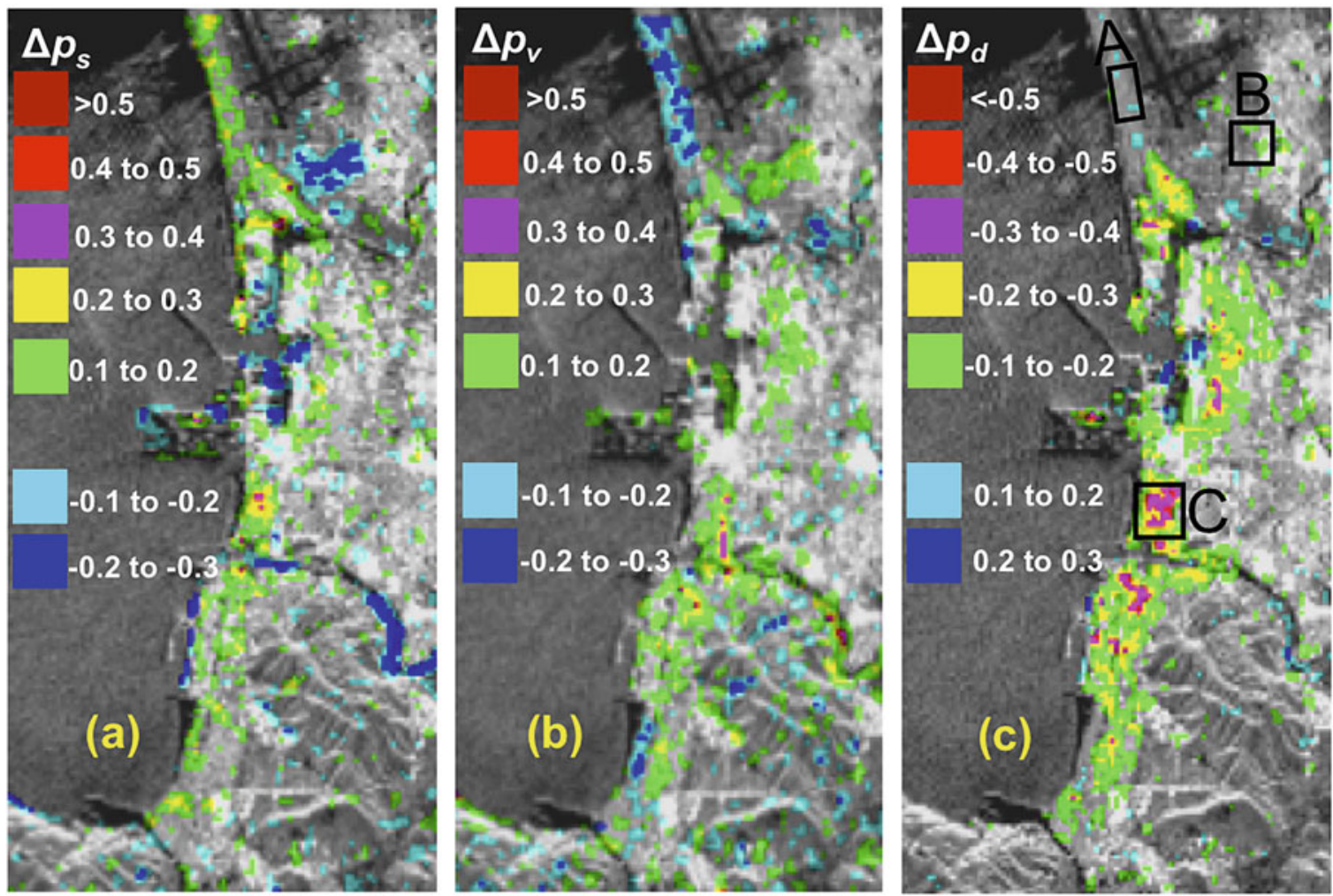

Fig. 3.39 The difference images in between pre-earthquake/tsunami (20090402) and post-earthquake/tsunami (20110408) for decomposition parameters (a) $\Delta p_{s}$, (b) $\Delta p_{v}$ and (c) $\Delta p_{d}$ are superimposed on $T P$ image of 20090402. Patch A shows the vegetation area on relatively flat surface areas, patch B represents the agricultural area over a relatively flat ground, and patch $\mathrm{C}$ illustrates urban areas of Ishinomaki city

Table 3.12 Statistics of the difference values of pixels for patches A, B and C in between pre- earthquake/tsunami (20090402) and postearthquake/tsunami (20110408) decomposition parameters

\begin{tabular}{l|l|l|l|l|}
\hline & Scattering component & Mean & Std. Dev. & Number of pixels \\
\hline \multirow{3}{*}{ Patch A } & $\Delta p_{s}$ & 0.107 & 0.070 \\
\cline { 2 - 4 } & $\Delta p_{v}$ & -0.154 & 0.066 \\
\cline { 2 - 4 } & $\Delta p_{d}$ & 0.047 & 0.054 \\
\cline { 2 - 4 } & $\Delta p_{c}$ & -0.000 & 0.015 \\
\hline \multirow{5}{*}{ Patch B } & $\Delta p_{s}$ & -0.222 & 0.050 \\
\cline { 2 - 4 } & $\Delta p_{v}$ & 0.145 & 0.046 \\
\cline { 2 - 4 } & $\Delta p_{d}$ & 0.061 & 0.031 \\
\cline { 2 - 4 } & $\Delta p_{c}$ & 0.016 & 0.008 \\
\hline \multirow{5}{*}{ Patch C } & $\Delta p_{s}$ & 0.136 & 0.132 \\
\cline { 2 - 4 } & $\Delta p_{v}$ & 0.150 & 0.093 \\
\cline { 2 - 4 } & $\Delta p_{d}$ & -0.280 & 0.075 \\
\cline { 2 - 4 } & $\Delta p_{c}$ & -0.006 & 0.018 \\
\hline
\end{tabular}


affected or damaged vegetation areas and increased in damaged or collapsed, deposited and/or floating (on bays water surface) urban blocks or man-made structures by the earthquake/tsunami; and (3) the double-bounce scattering is decreased in earthquake/tsunami collapsed or damaged urban areas and increased in areas with deposited (in agricultural fields or other type scattering dominated areas) wiped-out urban blocks or man-made structures, mainly caused by the force of tsunami. More detailed analysis and discussion are given in (Singh et al. 2013).

\subsubsection{Comparison with Single-/ Dual-Polarisation Data}

SAR images obtained from fixed single- and/or dualpolarisation sensors are independent of meteorological conditions, but are difficult to interpret and require tedious computational analyses for at most incomplete assessments only. A single observation using conventional SAR images makes it difficult to generate desirable images ready for direct visual interpretation. The features that can be identified in the fully polarimetric case cannot be distinguished in single and dual-pol images (see Fig. 3.38). However, high-resolution fully polarimetric SAR images are straightforward to identify the differences or damages between pre- and post-tsunami conditions of the affected regions.

\subsubsection{Discussion on the Role of Polarimetry, on the Maturity of the Application and Conclusions}

Since microwave radar remote sensing is a suitable tool for monitoring the near-real-time earthquake and tsunami damage at large scales, at anytime of day or night, its implementation becomes of vital relevance to governmental and other agencies for initiating swift and well-orchestrated rescue operations. In this showcase, we have explored the role of polarimetry in tsunami and earthquake disaster monitoring. Fully polarimetric high-resolution L-band image data sets with the implementation of the G4U scheme provide a straightforward simple tool for interpreting as well as identifying collapsed buildings caused by earthquake/tsunami disasters. This method also holds other types of natural (typhoon or tornado) and man-made disaster assessment application. It is found that the double-bounce scattering power is the most promising parameters to detect automated disaster-affected urban areas at pixel level. It is also observed that the very-high-resolution PolSAR images are required for superior urban area monitoring over the oriented urban blocks with respect to the illumination of radar.

\subsection{Summary (Table 3.13)}

Table 3.13 Summary of presented application, methods and preferred system configurations for monitoring and characterisation of agriculture and wetland scenarios

\begin{tabular}{|c|c|c|}
\hline Application & Methods and used frequency (P/L/C/X) & $\begin{array}{l}\text { Radar data preference/requirements/ } \\
\text { comments }\end{array}$ \\
\hline \multirow[t]{3}{*}{ Crop type mapping } & $\begin{array}{l}\text { Decision tree supervised classification applied to PolSAR } \\
\text { observables from incoherent decompositions, multitemporal data (C) }\end{array}$ & $\begin{array}{l}\text { Preferred frequency: } \mathrm{C}-\text { simpler } \\
\text { polarimetric modes could suffice }\end{array}$ \\
\hline & \multirow[t]{2}{*}{$\begin{array}{l}\text { Statistical classification applied to PolSAR backscattering, } \\
\text { multitemporal data (L/C) }\end{array}$} & $\begin{array}{l}\text { Need of multitemporal data: short revisit } \\
\text { time needed }\end{array}$ \\
\hline & & $\begin{array}{l}\text { Radiometric stability in time may be } \\
\text { needed }\end{array}$ \\
\hline $\begin{array}{l}\text { Soil moisture retrieval under } \\
\text { vegetation }\end{array}$ & PolSAR decomposition and inversion of scattering models (L/C) & Preferred frequency: L \\
\hline \multirow[t]{3}{*}{ Phenology monitoring } & \multirow[t]{3}{*}{ Hierarchical classification based on PolSAR observables (C) } & Short revisit time \\
\hline & & Radiometric stability in time \\
\hline & & $\begin{array}{l}\text { High-resolution and/or wide swath } \\
\text { desirable - compact-pol is a good trade- } \\
\text { off }\end{array}$ \\
\hline \multirow{4}{*}{$\begin{array}{l}\text { Wetland and peatland } \\
\text { delineation and } \\
\text { characterisation }\end{array}$} & Segmentation of the Shannon entropy image (C) & $\begin{array}{l}\text { Dual-pol could suffice (e.g. for } \\
\text { RADARSAT-2 and Sentinel-1) }\end{array}$ \\
\hline & PolSAR model-based decompositions (L/C/X) & Preferred frequencies: L/X \\
\hline & \multirow[t]{2}{*}{ Touzi decomposition (L) } & Preferred frequency: L \\
\hline & & Good SNR in HV needed \\
\hline $\begin{array}{l}\text { Effect of tsunami and } \\
\text { earthquakes (change } \\
\text { detection) }\end{array}$ & Four-component decomposition (L) & High resolution needed \\
\hline
\end{tabular}




\section{References}

Anguela TP, Zribi M, Baghdadi N, Loumange C (2010) Analysis of local variation of soil surface parameters with TerraSAR-X radar data over bare agricultural fields. IEEE Trans Geosci Remote Sens 48:874-881

Arii M, van Zyl JJ, Kim Y (2010) A general characterization for polarimetric scattering from vegetation canopies. IEEE Trans Geosci Remote Sens 48:3349-3357

Arii M et al (2011) Adaptive model-based decomposition of polarimetric SAR covariance matrices. IEEE Trans Geosci Remote Sens 49:1104-1113

Attema E, Ulaby FT (1978) Vegetation modelled as a water cloud. Radio Sci 13:357-364

Atwood D et al (2013) Proceedings of the 2013 POLinSAR workshop

Bach H, Mauser W (2003) Methods and examples for remote sensing data assimilation in land surface process modelling. IEEE Trans Geosci Remote Sens 41:1629-1637

Badhwar GD, Henderson KE (1981) Estimating development stages of corn from spectral data - an initial model. Agron J 73:748-755

Ballester-Berman JD, Lopez-Sanchez JM (2012) Time series of hybridpolarity parameters over agricultural crops. IEEE Geosci Remote Sens Lett 9:139-143

Ballester-Berman JD, Lopez-Sanchez JM, Fortuny-Guasch J (2005) Retrieval of biophysical parameters of agricultural crops using polarimetric SAR interferometry. IEEE Trans Geosci Remote Sens 43:683-694

Ban Y (2003) Synergy of multitemporal ERS-1 SAR and Landsat TM data for classification of agricultural crop. Can J Remote Sens 29:518-526

Barbier EB (1994) Valuing Environmental functions: tropical wetlands. Land Econ 70:155-173

Bernard R, Martin P, Thony JL, Vauclin M, Vidal-Madjar D (1982) C-band radar for determining surface soil moisture. Remote Sens Environ 12:189-200

Blaes X, Vanhalle L, Defourny P (2005) Efficiency of crop identification based on optical and SAR image time series. Remote Sens Environ 96:352-365

Bocco G, Mendoza M, Velázquez A (2001) Remote sensing and GIS-based regional geomorphological mapping - a tool for land use planning in developing countries. Geomorphology 39:211-219

Boerner WM et al (1998) Chapter 5: Polarimetry in radar remote sensing: basic and applied concepts. In: Ryerson RA (ed) Manual of remote sensing: principles and applications of imaging radar, vol 3. Wiley, pp 271-356

Bouman BAM, Hoekman D (1993) Multi-temporal, multi-frequency radar measurements of agricultural crops during the Agriscatt- 88 campaign in the Netherlands. Int J Remote Sens 14:1595-1614

Bouman BAM, Uenk D (1992) Crop classification possibilities with radar in ERS-1 and JERS-1 configuration. Remote Sensing of Environment 40:1-13

Bourgeau-Chavez LL et al (2005) Remote monitoring of regional inundation patterns and hydroperiod in the greater everglades using synthetic aperture radar. Wetlands 25:176-191

Bourgeau-Chavez LL, Riordan K, Powell RB, Miller N, Nowels M (2009) Improving wetland characterization with multi-sensor, multi-temporal SAR and optical/infrared data fusion. In: Jedlovec $\mathrm{G}$ (ed) Advances in geoscience and remote sensing. InTech, pp 679-708

Bouvet A, Le Toan T, Lam-Dao N (2009) Monitoring of the rice cropping system in the Mekong Delta using ENVISAT/ASAR dual polarization data. IEEE Trans Geosci Remote Sens 47:517-526

Brisco B, Brown RJ (1995) Multidate SAR/TM synergism for crop classification in Western Canada. Photogram Eng Remote Sens 61:1009-1014
Brisco B, Protz R (1980) Corn field identification accuracy using airborne radar imagery. Can J Remote Sens 6:15-24

Brisco B, Kapfer M, Hirose T, Tedford B, Liu J (2011) Evaluation of $\mathrm{C}$-band polarization diversity and polarimetry for wetland mapping. Can J Remote Sens 37:82-92

Bronstert A, Bardossy A (1999) The role of spatial variability of soil moisture for modelling surface runoff generation at the small catchment scale. Hydrol Earth Syst Sci 3:505-516

Bronstert A et al (2012) Potentials and constraints of different type of soil moisture observations for flood simulations in headwater catchments. Nat Hazar 60:879-914

Brook RK (2006) In: Riewe R, Oakes J (eds) Forest and tundra fires in the Hudson Bay Lowlands of Manitoba. Climate Change: Linking Traditional and Scientific Knowledge. Aboriginal Issues Press, Winnipeg

Brook RK, Kenkel NC (2002) A multivariate approach to vegetation mapping of Manitoba's Hudson Bay Lowlands. Int J Remote Sens 23:4761-4776

Brown RJ, Manore MJ, Poirier S (1992) Correlations between X-, Cand L-band imagery within an agricultural environment. Int $\mathbf{J}$ Remote Sens 13:1645-1661

Cardell-Oliver R, Kranz M, Smettem K, Mayer K (2005) A reactive soil moisture sensor network: design and field evaluation. Int J Distrib Sens Netw 1:149-162

Charbonneau FJ et al (2010) Compact polarimetry overview and applications assessment. Can J Remote Sens 36:298-315

Chen C, McNairn H (2006) A neural network integrated approach for rice crop monitoring. Int J Remote Sens 27:1367-1393

Chen KS, Huang WP, Tsay DH, Amar F (1996) Classification of multifrequency polarimetric SAR imagery using a dynamic learning neural network. IEEE Trans Geosci Remote Sens 34:814-820

Chini M, Pierdicca N, Emery WJ (2009) Exploiting SAR and VHR optical images to quantify damages caused by the 2003 Bam earthquake. IEEE Trans Geosci Remote Sens 47:145-152

Cloude SR (1988) Uniqueness of target decomposition theorems in radar polarimetry. In: Boerner WM et al (eds) Proceedings of NATO Advanced research workshop on direct and inverse methods in radar polarimetry, vol 350. Kluwer, pp 267-296

Cloude SR (2009) Polarisation: applications in remote sensing. Oxford University Press

Cloude SR, Papathanassiou KP (2003) Three-stage inversion process for polarimetric SAR interferometry. IEEE Proc Radar Sonar Navig 150:125-134

Cloude SR, Pottier E (1996) A review of target decomposition theorems in radar polarimetry. IEEE Trans Geosci Remote Sens 34:498-518

Cloude SR, Pottier E (1997) An entropy based classification scheme for land applications of polarimetric SAR. IEEE Trans Geosci Remote Sens 35:68-78

Cloude SR, Corr DG, Williams ML (2004) Target detection beneath foliage using polarimetric synthetic aperture radar interferometry. Waves Random Media 14:393-414

Cloude SR, Goodenough DG, Chen H (2012) Compact decomposition theory. IEEE Geosci Remote Sens Lett 9:28-32

Del Frate $\mathrm{F}$ et al (2004) Wheat cycle monitoring using radar data and a neural network trained by a model. IEEE Trans Geosci Remote Sens 42:35-44

Dobson MC, Pierce LE, Ulaby FT (1996) Knowledge-based land-cover classification using ERS-1/JERS-1 SAR composites. IEEE Trans Geosci Remote Sens 34:83-99

Dyke D, Wendy ES (2010) Permafrost and peatland evolution in the Northern Hudson Bay lowland, Manitoba. Arctic 63:429-441

EEA (2007) CLC2006 technical guidelines. Technical report, no. $17 / 2007$

Engdahl M, Borgeaud M (1998) ERS-1/2 Tandem interferometric coherence and agricultural crop height. In: Proceedings of retrieval 
of bio- and geo-physical parameters from SAR data for land applications workshop 121-127, ESA-ESTEC

Engdahl M, Borgeaud M, Rast M (2001) The use of ERS-1/2 tandem interferometric coherence in the estimation of agricultural crop heights. IEEE Trans Geosci Remote Sens 39:1799-1806

Entekabi D et al (2010) The soil moisture active passive (SMAP) mission. Proc IEEE 98:704-716

European Space Agency (2012) The earth observation handbook: 2012 special edition for Rio+20, Noordwijk

Evans TL, Costa M (2013) Landcover classification of the Lower Nhecolandia subregion of the Brazilian Pantanal Wetlands using ALOS/PALSAR, RADARSAT-2 and ENVISAT/ASAR imagery. Remote Sens Environ 128:118-137

Fatoyinbo T, Simard M (2011) Remote sensing of mangrove structure and biomass. In: Proceedings of the 2011 workshop on Tropical Wetland Ecosystems of Indonesia: Science Needs to Address Climate Change Adaptation and Mitigation

Ferrazzoli P et al (1992) Sensitivity of microwave measurements to vegetation biomass and soil moisture content: a case study. IEEE Trans Geosci Remote Sens 30:750-756

Ferrazzoli P, Paloscia S, Pampaloni P, Schiavon G, Sigismondi S, Solimini D (1997) The potential of multifrequency polarimetric SAR in assessing agricultural and arboreous biomass. IEEE Trans Geosci Remote Sens 35:5-17

Ferrazzoli P, Guerriero L, Schiavon G (1999) Experimental and model investigation on radar classification capability. IEEE Trans Geosci Remote Sens 37:960-968

Foody GM (1988) Crop classification from airborne synthetic aperture radar data. Int J Remote Sens 9:655-668

Foody GM, McCulloch MB, Yates WB (1994) Crop classification from C-Band polarimetric radar data. Int J Remote Sens 15:2871-2885

Fournier R, Grenier M, Lavoie A, Helie R (2007) Towards a strategy to implement the Canadian wetland inventory using satellite remote sensing. Can J Remote Sens 33:1-16

Fraser CJD, Roulet NT, Lafleur M (2001) Groundwater flow patterns in a large peatland. J Hydrol 246:142-154

Freeman A (2007) Fitting a two component scattering model to polarimetric SAR data from forests. IEEE Trans Geosci Remote Sens 45:2583-2592

Freeman A, Durden SL (1998) A Three-component scattering model for polarimetric SAR data. IEEE Trans Geosci Remote Sens 36:963-973

Freeman A, Villasenor J, Klein JD, Hoogeboom P, Groot J (1994) On the use of multi-frequency and polarimetric radar backscatter features for classification of agricultural crops. Int J Remote Sens 15:1799-1812

Fung AK (1994) Microwave scattering and emission models and their applications. Artech House, Boston

Gorham E (1995) Northern peatlands: role in the carbon budget and probable 7 responses to global warming. Ecol Appl 1:182-188

Grenier M, Demers AM, Labrecque S, Benoit M, Fournier R, Drolet B (2007) An object-based method to map wetland using RADARSAT1 and Landsat ETM images: test case on two sites in Quebec, Canada. Can J Remote Sens 33:28-45

Guindon B, Teillet PM, Goodenough DG, Palimaka JJ, Sieber A (1984) Evaluation of the crop classification performance of X, L and C-band SAR Imagery. Can J Remote Sens 10:4-16

Haboudane D, Miller J, Pattey E, Zarco-Tejada PJ, Strachan I (2004) Hyperspectral vegetation indices and novel algorithms for predicting green LAI of crop canopies: modeling and validation in the context of precision agriculture. Remote Sens Environ 90:337-352

Hajnsek I, Pottier E, Cloude SR (2003) Inversion of surface parameters from polarimetric SAR. IEEE Trans Geosci Remote Sens 4:727-744

Hajnsek I, Jagdhuber T, Schön H, Papathanassiou KP (2009) Potential of estimating soil moisture under vegetation cover by means of PolSAR. IEEE Trans Geosci Remote Sens 47:442-454
Henderson FM, Lewis AJ (eds) (1998) Manual of remote sensing: principles and applications of imaging radar, vol 2, 3rd edn. Wiley

Henderson FM, Lewis AJ (2008) Radar detection of wetland ecosystems: a review. Int J Remote Sens 29:5809-5835

Hess LL, Melack JM, Filoso S, Wang Y (1995) Delineation of inundated area and vegetation along the Amazon floodplain with SIR-C synthetic aperture radar. IEEE Trans Geosci Remote Sens 33:896-904

Hill MJ, Ticehurst CJ, Lee J-S, Grunes MR, Donald GE, Henry D (2005) Integration of optical and radar classifications for mapping pasture type in Western Australia. IEEE Trans Geosci Remote Sens 43:1665-1681

Hoekman DH, Vissers AM (2003) A new polarimetric classification approach evaluated for agricultural crops. IEEE Trans Geosci Remote Sens 41:2881-2889

Hoekman DH, Vissers AM, Tran TN (2011) Unsupervised fullpolarimetric SAR data segmentation as a tool for classification of agricultural areas. IEEE J Sel Topics Appl Earth Observ Remote Sens 4:402-411

Hong SH, Wdowinski S (2011) Evaluation of the quad-polarimetric RADARSAT-2 observations for the wetland InSAR application. Can J Remote Sens 37:484-492

Hong SH, Wdowinski S (2013) Double bounce component in crosspolarimetric SAR from new scattering target decomposition. In: Proceedings of the 2013 POLinSAR workshop

Hong SH et al (2010) Evaluation of TerraSAR-X observations for wetland InSAR application. IEEE Trans Geosci Remote Sens 48:864-873

Hubert-Moy L (2006) Wetlands. In: Geist H (ed) Our Earth's changing land: an encyclopedia of land-use and land-cover change, vol 2. Greenwood Press, pp 665-671

Hubert-Moy L, Michel K, Corpetti T, Clément B (2006) Object-oriented mapping and analysis of wetlands using SPOT 5 data. In: Proceedings of the IEEE Geoscience and Remote Sensing Symposium (IGARSS), pp 3447-3450

Huete AR et al (2006) Amazon rainforests green-up with sunlight in dry season. Geophys Res Lett 33

Huisman JA, Snepvangers JJJC, Bouten W, Heuvelink GBM (2003) Monitoring temporal development of spatial soil water content variation: comparison of ground penetration radar and time domain reflectometry. Vadose Zone J 2:519-529

Ingram HAP (1982) Size and shape in raised mire ecosystems: a geophysical model. Nature 297:300-303

Inoue $\mathrm{Y}$ et al (2002) Season-long daily measurements of multifrequency $(\mathrm{Ka}, \mathrm{Ku}, \mathrm{X}, \mathrm{C}$, and $\mathrm{L})$ and full-polarization backscatter signatures over paddy rice field and their relationship with biological variables. Remote Sens Environ 81:194-204

Jackson TJ (1993) Measuring surface soil moisture using passive microwave remote sensing. Hydrol Process 7:139-152

Jagdhuber T (2012) Soil parameter retrieval under vegetation cover using SAR polarimetry. PhD thesis, University of Potsdam. http:// opus.kobv.de/ubp/volltexte/2012/6051/

Jagdhuber T, Hajnsek I, Sauer S, Papathanassiou KP, Bronstert A (2012a) Soil moisture retrieval under forest using polarimetric decomposition techniques at P-band. In: Proceedings of European conference on Synthetic Aperture Radar (EUSAR)

Jagdhuber T, Hajnsek I, Papathanassiou KP, Bronstert A (2012b) Soil moisture retrieval under agricultural vegetation using fully polarimetric SAR. In: Proceedings of IEEE 2012 international symposium on Geoscience and Remote Sensing (IGARSS)

Jagdhuber T, Hajnsek I, Papathanassiou KP, Bronstert A (2013a) Soil moisture estimation under low vegetation cover using a multiangular polarimetric decomposition. IEEE Trans Geosci Remote Sens 51:2201-2215

Jagdhuber T, Hajnsek I, Papathanassiou KP (2013b) Refined soil moisture estimation by means of L-band polarimetry. In: Proceedings of 
IEEE international geoscience and remote sensing symposium (IGARSS)

Jagdhuber T, Hajnsek I, Papathanassiou KP (2013c) Polarimetric soil moisture retrieval at short wavelength. In: Proceedings of international workshop on Science and Applications of SAR Polarimetry and Polarimetric Interferometry (PolINSAR)

Jagdhuber T, Hajnsek I, Caputo M, Papathanassiou KP (2013d) Soil moisture estimation using dual-polarimetric coherent $(\mathrm{HH} / \mathrm{VV})$ TerraSAR-X and TanDEM-X data. In: Proceedings of TSX/TDX science meeting

Jagdhuber T, Hajnsek I, Papathanassiou KP (2014) An iterative generalized hybrid decomposition for soil moisture retrieval under vegetation cover using fully polarimetric SAR. IEEE J Sel Topic Appl Earth Observ Remote Sens. https://doi.org/10.1109/JSTARS. 2014.2371468

Jano AP, Jefferies RL, Rockwell R (1998) The detection of vegetational change by multitemporal analysis of LANDSAT data: The effects of goose foraging. J Ecol 86:93-99

Karam MA, Fung AK, Lang RH, Chauhan NS (1992) A microwave scattering model for layered vegetation. IEEE Trans Geosci Remote Sens 30:767-784

Katra I, Blumberg DG, Lavee H, Sarah P (2006) A method for estimating the spatial distribution of soil moisture of arid microenvironments by close range thermal infrared imaging. Int $\mathrm{J}$ Remote Sens 27:2599-2611

Kerr Y, Waldteufel P, Wigneron J-P, Martinuzzi J-M, Font J, Berger M (2001) Soil moisture retrieval from space: the soil moisture and ocean salinity (SMOS) mission. IEEE Trans Geosci Remote Sens 39:1729-1735

Koay J-Y et al (2007) Paddy fields as electrically dense media: theoretical modeling and measurement comparisons. IEEE Trans Geosci Remote Sens 45:2837-2849

Koch M, Schmid T, Reyes M, Gumuzzio J (2012) Evaluating full polarimetric $\mathrm{C}$ - and $\mathrm{L}$-band data for mapping wetland conditions in a semi-arid environment in central Spain. IEEE J Sel Topic Appl Earth Observ Remote Sens 5:1033-1044

Koyama CN, Schneider K (2011) Soil moisture retrieval under vegetation using polarized PALSAR data. In: Proceedings of IEEE 2011 international symposium on Geoscience and Remote Sensing (IGARSS)

Krajewski WF et al (2006) A remote sensing observatory for hydrologic sciences: a genesis for scaling to continental hydrology. Water Resour Res 42. https://doi.org/10.1029/2005WR004435

Kurosu T, Fujita M, Chiba K (1995) Monitoring of rice crop growth from space using the ERS-1 C-band SAR. IEEE Trans Geosci Remote Sens 33:1092-1096

Lang MW, Kasischke ES (2008) Using C-band synthetic aperture radar data to monitor forested, wetland hydrology in Maryland's coastal plain, USA. IEEE Trans Geosci Remote Sens 46:535-546

Lang RH, Sidhu JS (1983) Electromagnetic backscattering from a layer of vegetation: a discrete approach. IEEE Trans Geosci Remote Sens 21:62-71

Lasne Y, Paillou P, August-Bernex T, Ruffie G, Grandjean G (2004) A phase signature for detecting wet subsurface structures using polarimetric L-band SAR. IEEE Trans Geosci Remote Sens 42:1683-1694

Le Toan T, Laur H, Mougin E, Lopes A (1989) Multitemporal and dual polarization observations of agricultural vegetation covers by X-band SAR images. IEEE Trans Geosci Remote Sens 27:709-718

Le Toan $T$ et al (1997) Rice crop mapping and monitoring using ERS-1 data based on experiment and modeling results. IEEE Trans Geosci Remote Sens 35:41-56

Lee J-S, Ainsworth TL (2011) The effect of orientation angle compensation on coherency matrix and polarimetric target decompositions. IEEE Trans Geosci Remote Sens 49:53-64

Lee J-S, Pottier E (2009) Polarimetric radar imaging: from basics to applications. CRC Press, Taylor \& Francis
Lee JS, Grunes R, Kwok R (1994) Classification of multi-look polarimetric SAR imagery based on complex Wishart distribution. Int J Remote Sens 15:2299-2311

Lee JS, Grunes MR, Ainsworth TL, Du LJ, Schuler DL, Cloude SR (1999) Unsupervised classification using polarimetric decomposition and the complex Wishart classifier. IEEE Trans Geosci Remote Sens 37:2249-2258

Lee J-S, Grunes MR, Pottier E (2001) Quantitative comparison of classification capability: fully polarimetric versus dual and singlepolarization SAR. IEEE Trans Geosci Remote Sens 39:2343-2351

Lee JS, Grunes MR, Pottier E, Ferro-Famil L (2004) Unsupervised terrain classification preserving polarimetric scattering characteristics. IEEE Trans Geosci Remote Sens 42:722-731

Liao J, Wang Q (2010) Wetland characterization and classification using polarimetric RADARSAT-2 data. Proc SPIE 7841. https://doi.org/ $10.1117 / 12.873258$

Lloyd D (1990) A phenological classification of terrestrial vegetation cover using shortwave vegetation index imagery. Int J Remote Sens 11:2269-2279

Lopez-Sanchez JM, Ballester-Berman JD (2009) Potentials of polarimetric SAR interferometry for agriculture monitoring. Radio Sci 44. https://doi.org/10.1029/2008RS004078

Lopez-Sanchez JM, Cloude SR, Ballester-Berman JD (2012a) Rice phenology monitoring by means of SAR polarimetry at X-band. IEEE Trans Geosci Remote Sens 50:2695-2709

Lopez-Sanchez JM, Hajnsek I, Ballester-Berman JD (2012b) First demonstration of agriculture height retrieval with PolInSAR airborne data. IEEE Geosci Remote Sens Lett 9:242-246

Lopez-Sanchez JM, Vicente-Guijalba F, Ballester-Berman JD, Cloude SR (2014) Polarimetric response rice fields at C-band: analysis and phenology retrieval. IEEE Trans Geosci Remote Sens 52:2977-2993

Löw A, Ludwig R, Mauser W (2006) Derivation of surface soil moisture from ENVISAT ASAR wide swath and image mode data in agricultural areas. IEEE Trans Geosci Remote Sens 44:889-899

Lucas RM et al (2007) The potential of L-band SAR for quantifying mangrove characteristics and change: case studies from the tropics. Aquat Conserv Mar Freshw Ecosyst 17:245-264

Maltby E (ed) (2009) The functional assessment wetland ecosystems: towards evaluation of ecosystem services. Woodhead Publ, Boston

Marechal C, Pottier E, Hubert-Moy L, Corgne S, Allain-Bailhache S, Meric S (2010) Spaceborne fully polarimetric time-series datasets for land cover analysis. In: Proceedings of the IEEE International Geoscience and Remote Sensing Symposium (IGARSS), pp 859-862

Marechal C, Pottier E, Hubert-Moy L, Rapinel S (2012) One year wetland survey investigations from quad-pol RADARSAT-2 timeseries SAR images. Can J Remote Sens 38:240-252

Matsuoka M, Koshimura S (2010) Tsunami damaged area estimation for the 2010 Maule, Chile earthquake using ASTER DEM and PALSAR images on the geo grid. In: 8th international workshop on Remote Sensing and Disaster Response, Tokyo, Japan

McNairn H, van der Sanden JJ, Brown RJ, Ellis J (2000) The potential of RADARSAT-2 for crop mapping and assessing crop condition. In: Proceedings of the second international conference on Geospatial Information in Agriculture and Forestry

McNairn H, Champagne C, Shang J, Holmstrom D, Reichert G (2009a) Integration of optical and synthetic aperture radar (SAR) imagery for delivering operational annual crop inventories. ISPRS J Photogram Remote Sens 64:434-449

McNairn H, Shang J, Jiao X, Champagne C (2009b) The contribution of ALOS PALSAR multipolarization and polarimetric data to crop classification. IEEE Trans Geosci Remote Sens 47:3981-3992

Meehl GA et al (2007) Global climate projections. In: Climate change 2007: the physical science basis. Contributions of Working Group I to the Fourth Assessment Report of the Intergovernmental Panel on Climate Change. Cambridge University Press, Cambridge 
Meier U (ed) (2001) Growth stages of mono- and dicotyledonous plants. BBCH Monograph. http://www.jki.bund.de/fileadmin/dam_ uploads/_veroeff/bbch/BBCH-Skala_englisch.pdf

Mérot P et al (2006) A method for improving the management of controversial wetland. Environ Manag 37:258-270

Merz B, Plate EJ (1997) An analysis of the effects of spatial variability of soil and soil moisture on runoff. Water Resour Res 33:2909-2922

Minet J, Laloy E, Lambot S, Vanclooster M (2011) Effect of highresolution spatial soil moisture variability on simulated runoff response using a distributed hydrological model. Hydrol Earth Syst Sci 15:1323-1338

Moran MS, Peters-Lidard CD, Watts JM, McElroy S (2004) Estimating soil moisture at the watershed scale with satellite-based radar and land surface models. Can J Remote Sens 30:805-826

Moran MS et al (2012) A RADARSAT-2 quad-polarized time series for monitoring crop and soil conditions in Barrax, Spain. IEEE Trans Geosci Remote Sens 50:1057-1070

Nellis MD, Price KP, Rundquist D (2009) Remote sensing of cropland agriculture. Papers in Natural Resources 217

Neumann M et al (2009) A general model-based polarimetric decomposition scheme for vegetated areas. In: Proceedings of the 2009 POLInSAR workshop

Neumann M, Ferro-Famil L, Reigber A (2010) Estimation of forest structure, ground and canopy layer characteristics from multibaseline polarimetric interferometric SAR data. IEEE Trans Geosci Remote Sens 48:1086-1104

Ozesmi SL, Bauer M (2002) Satellite remote sensing of wetlands. Wetl Ecol Manag 10:381-402

Park SE, Kimb D, Lee H-S, Moon WM, Wagner W (2010) Tidal wetland monitoring using polarimetric synthetic aperture radar. In: Wagner W, Székely B (eds) ISPRS TC VII symposium - 100 years ISPRS, vol XXXVIII. IAPRS, Vienna

Patel P, Srivastava HS, Navalgund RR (2009) Use of synthetic aperture radar polarimetry to characterize wetland targets of Koladeo National Park, Bharatpur, India. Curr Sci 97:529-537

Pierce LE, Ulaby FT, Sarabandi K, Dobson MC (1994) Knowledgebased classification of polarimetric SAR images. IEEE Trans Geosci Remote Sens 32:1081-1086

Pope KO et al (1994) Radar remote-sensing of forest and wetland ecosystems in the Central-American tropics. Remote Sens Environ 48:205-219

Pope KO et al (1997) Detecting seasonal flooding cycles in marshes of the Yucatan Peninsula with SIR-C polarimetric radar imagery. Remote Sens Environ 59:157-166

Proisy C et al (2000) Interpretation of polarimetric radar signatures of mangrove forests. Remote Sens Environ 71:56-66

Quegan S, Le Toan T, Skriver H, Gomez-Dans J, Gonzalez-Sampedro MC, Hoekman DH (2003) Crop classification with multitemporal polarimetric SAR data. In: Proceedings of POLinSAR2003 workshop, Frascati, 2003

Raney RK (2007) Hybrid-polarity SAR architecture. IEEE Trans Geosci Remote Sens 45:3397-3404

Reed BC et al (1994) Measuring phenological variability from satellite imagery. J Veg Sci 5:703-714

Robinson DA, Jones SB, Wraith JM, Or D, Friedman SP (2003) A review of advances in dielectric and electrical conductivity measurement in soils using time domain reflectometry. Vadose Zone J 2:444-475

Robinson DA et al (2008) Soil moisture measurement for ecological and hydrological watershed-scale observatories: A review. Vadose Zone J 7:358-389

Rosenqvist A, Shimada M, Ito N, Watanabe M (2007) ALOS PALSAR: a pathfinder mission for global-scale monitoring of the environment. IEEE Trans Geosci Remote Sens 45:3307-3316

Rulequest Research (2008) Rulequest research, data mining tools See5 and C5.0. http://www.rulequest.com/see5-info.html
Sato A et al (2012a) Four-component scattering power decomposition with extended volume scattering model. IEEE Geosci Remote Sens Lett 9:166-170

Sato M, Chen S-W, Makoto S (2012b) Polarimetric SAR analysis of tsunami damage following the March 11, 2011 East Japan earthquake. Proc IEEE 100:2861-2875

Schmitt A, Brisco B (2013) Wetland monitoring using the curveletbased change detection method on polarimetric SAR imagery. Water 5:1036-1051

Schmitt A, Brisco B, Kaya S, Murnaghan K (2012) Polarimetric change detection for wetlands, remote sensing and hydrology. Proc IAHS 352:375-379

Schotten CGJ, Van Rooy WWL, Janssen LLF (1995) Assessment of the capabilities of multi-temporal ERS-1 SAR data to discriminate between agricultural crops. Int J Remote Sens 16:2619-2637

Shalaby A, Tateishi R (2007) Remote sensing and GIS for mapping and monitoring land cover and land-use changes in the Northwestern coastal zone of Egypt. Appl Geogr 27:28-41

Singh G, Yamaguchi Y, Park S-E (2012) General four-component scattering power decomposition with unitary transformation of coherency matrix. IEEE Trans Geosci Remote Sens 50

Singh G, Yamaguchi Y, Boerner WM, Park S-E (2013) Monitoring of the 2011 March 11 Off-Tohoku 9.0 earthquake with super-tsunami disaster by implementing fully polarimetric high resolution PolSAR techniques. Proc IEEE 101:831-846

Skriver H (2001) Land-cover map information from polarimetric SAR using knowledge-based techniques. In: Proceedings of 3rd international symposium on Retrieval of Bio- and Geophysical Parameters from SAR data for Land Applications, 2001

Skriver H (2012) Crop classification by multitemporal C- and L-band single- and dual-polarization and fully polarimetric SAR. IEEE Trans Geosci Remote Sens 50:2138-2149

Skriver H, Svendsen MT, Thomsen A (1999) Multitemporal C- and L-band polarimetric signatures of crops. IEEE Trans Geosci Remote Sens 37:2413-2429

Skriver H, Mattia F, Satalino G, Balenzano A, Pauwels VRN, Verhoest N, Davidson M (2011) Crop classification using shortrevisit multitemporal SAR data. IEEE J Sel Topics Appl Earth Observ Remote Sens 4:423-431

Slatton KC et al (1996) Modeling wetland vegetation using polarimetric SAR

Sokol J, McNairn H, Pultz T (2004) Case studies demonstrating hydrological applications of C-band multi-polarized and polarimetric SAR. Can J Remote Sens 30:470-483

Souyris JC, Imbo P, Fjortoft R, Mingot S, Lee J-S (2005) Compact polarimetry based on symmetry properties of geophysical media: The pi/4-mode. IEEE Trans Geosci Remote Sens 43:634-646

Technical Report Y-87-1 (1987) Environmental laboratory, corps of engineers wetlands delineation manual. U.S. Army Engineer Waterways Experiment Station, Vicksburg

Thomson KPB, Edwards G, Landry R, Jaton A, Cadieux SP, Gwyn QHJ (1990) SAR Applications in agriculture: multiband correlation and segmentation. Can J Remote Sens 16:47-54

Tilton JC, Hollinger SE (1982) Estimating crop development stages from multispectral data. LARS technical report, Purdue University/ Laboratory for Applications of Remote Sensing

Toutin T, Wang H, Chomaz P, Pottier E (2013) Orthorectification of full-polarimetric RADARSAT-2 data using accurate lidar DSM. IEEE Trans Geosci Remote Sens 51

Touzi R (2007a) Target scattering decomposition in terms of rollinvariant target parameters. IEEE Trans Geosci Remote Sens 45

Touzi R (2007b) Speckle effect on polarimetric target scattering decomposition of SAR imagery. Can J Remote Sens 33:60-68

Touzi R (2013) Compact-hybrid versus linear-dual and fully polarimetric SAR. In: Proceedings of POLinSAR workshop 
Touzi R, Gosselin G (2010) Peatland subsurface water flow monitoring using polarimetric L-band PALSAR. In: Proceedings of the IEEE International Geoscience and Remote Sensing Symposium (IGARSS), pp 750-753

Touzi R, Shimada M (2009) Calibration of polarimetric PALSAR. IEEE Trans Geosci Remote Sens 47:3911-3913

Touzi R, Boerner WM, Lee J-S, Luneberg E (2004) A review of polarimetry in the context of synthetic aperture radar: concepts and information extraction. Can J Remote Sens 30:380-407

Touzi R, Deschamps A, Rother G (2007) Wetland characterization using polarimetric RADARSAT-2 capability. Can J Remote Sens 33:56-67

Touzi R, Deschamps A, Rother G (2009) Phase of target scattering for wetland characterization using polarimetric C-band SAR. IEEE Trans Geosci Remote Sens 47:3241-3261

Treuhaft RN, Siqueira PR (2000) Vertical structure of vegetated land surfaces from interferometric and polarimetric data. Radio Sci 35:141-177

Treuhaft RN, Madsen SN, Moghaddam M, van Zyl JJ (1996) Vegetation characteristics and underlying topography from interferometric radar. Radio Sci 31:1449-1485

Trisasongko BH (2009) Tropical mangrove mapping using fullypolarimetric radar data. ITB J Sci 41:98-109

Tucker CJ (1979) Red and photographic infrared linear combinations for monitoring vegetation. Remote Sens Environ 8:127-150

Turkar V, Rao YS (2011) Analysis of multi-frequency SIR-C data over India. In: Proceeding of the 2011 international conference ICWET

Ulaby FT, Elachi C (1990) Radar polarimetry for geoscience applications. Artech House, Norwood

Ulaby FT, Wilson EA (1985) Microwave attenuation properties of vegetation canopies. IEEE Trans Geosci Remote Sens 23:746-753

Ulaby FT, Moore RK, Fung AK (1982) Microwave remote sensing: active and passive: Volume II: Radar remote sensing and surface scattering and emission theory. Artech House, Boston

Ulaby FT, Held DN, Dobson MC, McDonald KC, Senior TBA (1987) Relating polarization phase difference of SAR signals to scene properties. IEEE Trans Geosci Remote Sens 25:83-92

Ullmann T et al (2012) Application of quadpolarimetric TerraSAR-X data for landcover characterization in tropical regions - a case study in south Kalimantan, Indonesia. In: Proceedings of the IEEE International Geoscience and Remote Sensing Symposium, pp 5133-5136

United Nations Population Fund (2011) The state of world population 2011, New York

Van Zyl JJ (1989) Unsupervised classification of scattering behavior using radar polarimetry data. IEEE Trans Geosci Remote Sens $27: 36-45$
Van Zyl JJ (1992) Application of Cloude's target decomposition theorem to polarimetric imaging radar data. In: Mott $\mathrm{H}$, Boerner WM (eds) Proceedings of SPIE, vol 1748, San Diego, pp 184-191

Van Zyl JJ, Kim Y, Arii M (2008) Requirements for model-based polarimetric decompositions. In: Proceedings of IEEE 2008 International Symposium on Geoscience and Remote Sensing (IGARSS)

van Zyl JJ et al (2011) Model-based decomposition of polarimetric SAR covariance matrices constrained for nonnegative eigenvalues. IEEE Trans Geosci Remote Sens 49:3452-3459

Wagner W et al (2007) Operational readiness of microwave remote sensing of soil moisture for hydrologic applications. Nord Hydrol 28:1-20

Wagner W, Pathe C, Doubkova M et al (2008) Temporal stability of soil moisture and radar backscatter observed by the advanced synthetic aperture radar (ASAR). Sensors 8:1174-1197

Wegmüller U, Werner CL (1997) Retrieval of vegetation parameters with SAR interferometry. IEEE Trans Geosci Remote Sens 35:18-24

Western AW, Grayson RB, Blöschl G (2002) Scaling of soil moisture: a hydrologic perspective. Annu Rev Earth Planet Sci 30:149-180

Wiener N (1930) Generalized harmonic analysis. Acta Mathematica $5: 118-258$

Xiaoying J (2009) Segmentation-based image processing system. US patent 20090123070, filed Nov. 14, 2007, and issued May 14, 2009

Yajima Y, Yamaguchi Y, Sato R, Yamada H, Boerner W-M (2008) PolSAR image analysis of wetlands using a modified fourcomponent scattering power decomposition. IEEE Trans Geosci Remote Sens 46:1667-1673

Yamaguchi Y (2012) Disaster monitoring by fully polarimetric SAR data acquired with ALOS-PALSAR. Proc IEEE 100:2851-2860

Yamaguchi Y et al (2005) Four-component scattering model for polarimetric SAR image decomposition. IEEE Trans Geosci Remote Sens 43:1699-1706

Yamaguchi Y, Yajima Y, Yamada H (2006) A four component decomposition of PolSAR images based on the coherency matrix. IEEE Geosci Remote Sens Lett 3:292-296

Yamaguchi Y et al (2011) Four-component scattering power decomposition with rotation of coherency matrix. IEEE Trans Geosci Remote Sens 49:2251-2258

Zoltai SC, Vitt DH (1995) Canadian wetlands: environmental gradients and classification. Vegetation 118:131-137

Zreda M, Desilets D, Ferre TPA, Scott RL (2008) Measuring soil moisture content non-invasively at intermediate spatial scale using cosmic ray neutrons. Geophys Res Lett 35:1-5

Open Access This chapter is licensed under the terms of the Creative Commons Attribution 4.0 International License (http://creativecommons.org/ licenses/by/4.0/), which permits use, sharing, adaptation, distribution and reproduction in any medium or format, as long as you give appropriate credit to the original author(s) and the source, provide a link to the Creative Commons license and indicate if changes were made.

The images or other third party material in this chapter are included in the chapter's Creative Commons license, unless indicated otherwise in a credit line to the material. If material is not included in the chapter's Creative Commons license and your intended use is not permitted by statutory regulation or exceeds the permitted use, you will need to obtain permission directly from the copyright holder.

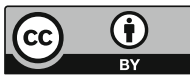

